quatrième série-tome 45 fascicule 1 janvier-février 2012

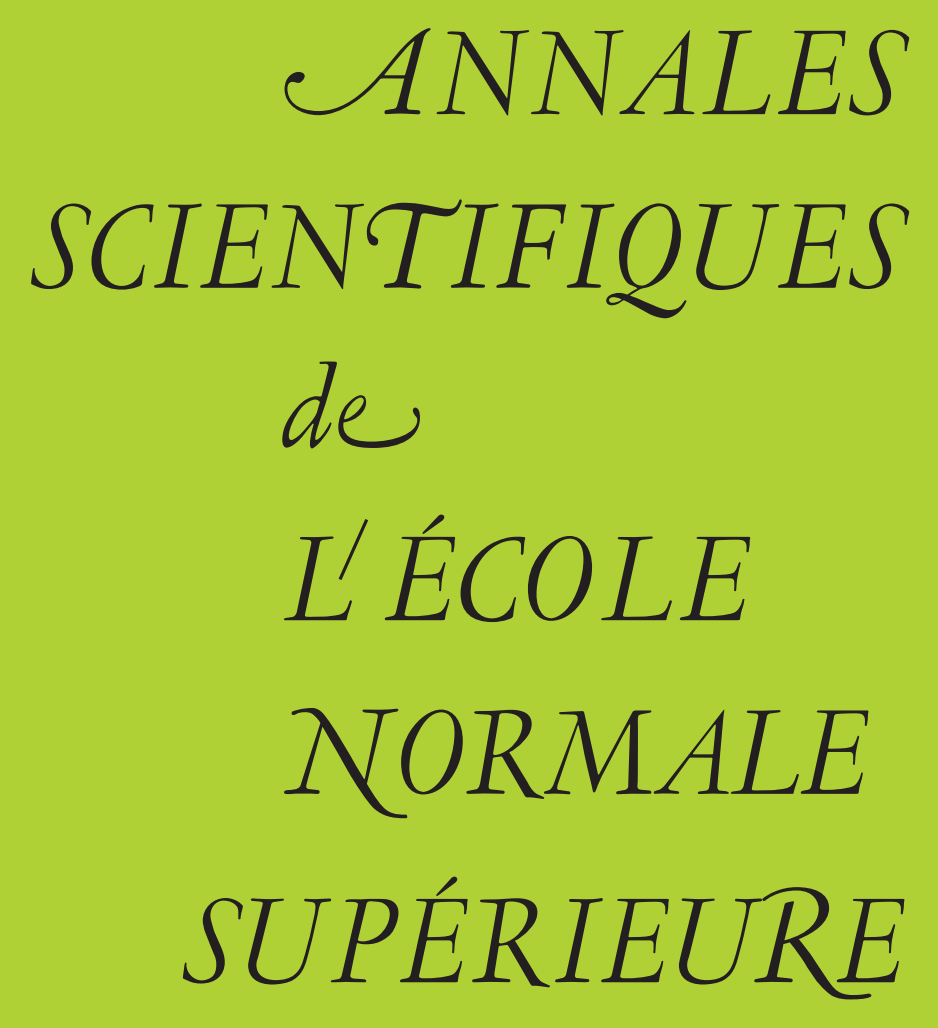

Reto MÜLLER

Ricci flow coupled with barmonic map flow 
Ann. Scient. Éc. Norm. Sup.

$4^{\mathrm{e}}$ série, t. 45, 2012, p. 101 à 142

\title{
RICCI FLOW COUPLED WITH HARMONIC MAP FLOW
}

\author{
BY RETO MÜLLER
}

Abstract. - We investigate a coupled system of the Ricci flow on a closed manifold $M$ with the harmonic map flow of a map $\phi$ from $M$ to some closed target manifold $N$,

$$
\frac{\partial}{\partial t} g=-2 \mathrm{Rc}+2 \alpha \nabla \phi \otimes \nabla \phi, \quad \frac{\partial}{\partial t} \phi=\tau_{g} \phi
$$

where $\alpha$ is a (possibly time-dependent) positive coupling constant. Surprisingly, the coupled system may be less singular than the Ricci flow or the harmonic map flow alone. In particular, we can always rule out energy concentration of $\phi$ a-priori by choosing $\alpha$ large enough. Moreover, it suffices to bound the curvature of $(M, g(t))$ to also obtain control of $\phi$ and all its derivatives if $\alpha \geq \underline{\alpha}>0$. Besides these new phenomena, the flow shares many good properties with the Ricci flow. In particular, we can derive the monotonicity of an energy, an entropy and a reduced volume functional. We then apply these monotonicity results to rule out non-trivial breathers and geometric collapsing at finite times.

RÉSUMÉ. - Nous étudions un système d'équations consistant en un couplage entre le flot de Ricci et le flot harmonique d'une fonction $\phi$ allant de $M$ dans une variété cible $N$,

$$
\frac{\partial}{\partial t} g=-2 \mathrm{Rc}+2 \alpha \nabla \phi \otimes \nabla \phi, \quad \frac{\partial}{\partial t} \phi=\tau_{g} \phi
$$

où $\alpha$ est une constante de couplage strictement positive (et pouvant dépendre du temps). De manière surprenante, ce système couplé peut être moins singulier que le flot de Ricci ou le flot harmonique si ceux-ci sont considérés de manière isolée. En particulier, on peut toujours montrer que la fonction $\phi$ ne se concentre pas le long de ce système à condition de prendre $\alpha$ assez grand. De plus, il est suffisant de borner la courbure de $(M, g(t))$ le long du flot pour obtenir le contrôle de $\phi$ et de toutes ses dérivées si $\alpha \geq \underline{\alpha}>0$. À part ces phénomènes nouveaux, ce flot possède certaines propriétés analogues à celles du flot de Ricci. En particulier, il est possible de montrer la monotonie d'une énergie, d'une entropie et d'une fonctionnelle volume réduit. On utilise la monotonie de ces quantités pour montrer l'absence de solutions en « accordéon » et l'absence d'effondrement en temps fini le long du flot. 


\section{Introduction and main results}

Let $\left(M^{m}, g\right)$ and $\left(N^{n}, \gamma\right)$ be smooth Riemannian manifolds without boundary. According to Nash's embedding theorem [30] we can assume that $N$ is isometrically embedded into Euclidean space $\left(N^{n}, \gamma\right) \hookrightarrow \mathbb{R}^{d}$ for a sufficiently large $d$. If $e_{N}: N \rightarrow \mathbb{R}^{d}$ denotes this embedding, we identify maps $\phi: M \rightarrow N$ with $e_{N} \circ \phi: M \rightarrow \mathbb{R}^{d}$, such maps may thus be written as $\phi=\left(\phi^{\lambda}\right)_{1 \leq \lambda \leq d}$. Harmonic maps $\phi: M \rightarrow N$ are critical points of the energy functional

$$
E(\phi)=\int_{M}|\nabla \phi|^{2} d V
$$

Here, $|\nabla \phi|^{2}:=2 e(\phi)=g^{i j} \nabla_{i} \phi^{\lambda} \nabla_{j} \phi^{\lambda}$ denotes the local energy density, where we use the convention that repeated Latin indices are summed over from 1 to $m$ and repeated Greek indices are summed over from 1 to $d$. We often drop the summation indices for $\phi$ when clear from the context. Harmonic maps generalize the concept of harmonic functions and in particular include closed geodesics and minimal surfaces.

To study the existence of a harmonic map $\phi$ homotopic to a given map $\phi_{0}: M \rightarrow N$, Eells and Sampson [13] proposed to study the $L^{2}$-gradient flow of the energy functional (1.1),

$$
\frac{\partial}{\partial t} \phi=\tau_{g} \phi, \quad \phi(0)=\phi_{0},
$$

where $\tau_{g} \phi$ denotes the intrinsic Laplacian of $\phi$, often called the tension field of $\phi$. They proved that if $N$ has non-positive sectional curvature there always exists a unique, global, smooth solution of (1.2) which converges smoothly to a harmonic map $\phi_{\infty}: M \rightarrow N$ homotopic to $\phi_{0}$ as $t \rightarrow \infty$ suitably. On the other hand, without an assumption on the curvature of $N$, the solution might blow up in finite or infinite time. Comprehensive surveys about harmonic maps and the harmonic map flow are given in Eells-Lemaire [11, 12], Jost [18] and Struwe [40]. The harmonic map flow was the first appearance of a nonlinear heat flow in Riemannian geometry. Today, geometric heat flows have become an intensely studied topic in geometric analysis.

Another fundamental problem in differential geometry is to find canonical metrics on Riemannian manifolds, for example metrics with constant curvature in some sense. Using the idea of evolving an object to such an ideal state by a nonlinear heat flow, Richard Hamilton [15] introduced the Ricci flow in 1982. His idea was to smooth out irregularities of the curvature by evolving a given Riemannian metric $g$ on a manifold $M$ with respect to the nonlinear weakly parabolic equation

$$
\frac{\partial}{\partial t} g=-2 \mathrm{Rc}, \quad g(0)=g_{0},
$$

where Rc denotes the Ricci curvature of $(M, g)$. Strictly speaking, the Ricci flow is not the gradient flow of a functional $\mathcal{F}(g)=\int_{M} F\left(\partial^{2} g, \partial g, g\right) d V$, but in 2002, Perelman [31] showed that it is gradient-like nevertheless. He presented a new functional which may be regarded as an improved version of the Einstein-Hilbert functional $E(g)=\int_{M} R d V$, namely

$$
\mathcal{F}(g, f):=\int_{M}\left(R+|\nabla f|^{2}\right) e^{-f} d V
$$

$4^{\mathrm{e}}$ SÉRIE - TOME $45-2012-\mathrm{N}^{\circ} 1$ 
The Ricci flow can be interpreted as the gradient flow of $\mathcal{F}$ modulo a pull-back by a family of diffeomorphisms. Hamilton's Ricci flow has a successful history. Most importantly, Perelman's work [31, 33] led to a completion of Hamilton's program [16] and a complete proof of Thurston's geometrization conjecture [42] and (using a finite extinction result from Perelman [32] or Colding and Minicozzi [8, 9]) of the Poincaré conjecture [34]. Introductory surveys on the Ricci flow and Perelman's functionals can be found in the books by Chow and Knopf [6], Chow, Lu and Ni [7], Müller [27] and Topping [43]. More advanced explanations of Perelman's proof of the two conjectures are given in Cao and Zhu [3] Chow et al. [4, 5], Kleiner and Lott [19] and Morgan and Tian [25, 26]. A good survey on Perelman's work is also given in Tao [41].

The goal of this article is to study a coupled system of the two flows (1.2) and (1.3). Again, we let $\left(M^{m}, g\right)$ and $\left(N^{n}, \gamma\right)$ be smooth manifolds without boundary and with $\left(N^{n}, \gamma\right) \hookrightarrow \mathbb{R}^{d}$. Throughout this article, we will assume in addition that $M$ and $N$ are compact, hence closed. However, many of our results hold for more general manifolds.

Let $g(t)$ be a family of Riemannian metrics on $M$ and $\phi(t)$ a family of smooth maps from $M$ to $N$. We call $(g(t), \phi(t))_{t \in[0, T)}$ a solution to the coupled system of Ricci flow and harmonic map heat flow with coupling constant $\alpha(t)$, the $(R H)_{\alpha}$ flow for short, if it satisfies

$(R H)_{\alpha}$

$$
\left\{\begin{array}{l}
\frac{\partial}{\partial t} g=-2 \mathrm{Rc}+2 \alpha \nabla \phi \otimes \nabla \phi, \\
\frac{\partial}{\partial t} \phi=\tau_{g} \phi .
\end{array}\right.
$$

Here, $\tau_{g} \phi$ denotes the tension field of the map $\phi$ with respect to the evolving metric $g$, and $\alpha(t) \geq 0$ denotes a (time-dependent) coupling constant. Finally, $\nabla \phi \otimes \nabla \phi$ has the components $(\nabla \phi \otimes \nabla \phi)_{i j}=\nabla_{i} \phi^{\lambda} \nabla_{j} \phi^{\lambda}$. In particular, $|\nabla \phi|^{2}$ as defined above is the trace of $\nabla \phi \otimes \nabla \phi$ with respect to $g$.

The special case where $N \subseteq \mathbb{R}$ and $\alpha \equiv 2$ was studied by List [22], his motivation coming from general relativity and the study of Einstein vacuum equations. Moreover, List's flow also arises as the Ricci flow of a warped product, see [28, Lemma A.3]. After completion of this work, we learned that another special case of $(R H)_{\alpha}$ with $N \subseteq S L(k \mathbb{R}) / S O(k)$ arises in the study of the long-time behavior of certain Type III Ricci flows, see Lott [23] and a recent paper of Williams [44] for details and explicit examples.

The paper is organized as follows. In order to get a feeling for the flow, we first study explicit examples of solutions of $(R H)_{\alpha}$ as well as soliton solutions which are generalized fixed points modulo diffeomorphisms and scaling. The stationary solutions of $(R H)_{\alpha}$ satisfy $\mathrm{Rc}=\alpha \nabla \phi \otimes \nabla \phi$, where $\phi$ is a harmonic map. To prevent $(M, g(t))$ from shrinking to a point or blowing up, it is convenient to introduce a volume-preserving version of the flow.

In Section 3, we prove that for constant coupling functions $\alpha(t) \equiv \alpha>0$ the $(R H)_{\alpha}$ flow can be interpreted as a gradient flow for an energy functional $\mathcal{F}_{\alpha}(g, \phi, f)$ modified by a family of diffeomorphisms generated by $\nabla f$. If $(g(t), \phi(t))$ solves $(R H)_{\alpha}$ and $e^{-f}$ is a solution to the adjoint heat equation under the flow, then $\mathcal{F}_{\alpha}$ is non-decreasing and constant if and only if $(g(t), \phi(t))$ is a gradient steady soliton. In the more general case where $\alpha(t)$ is a positive function, the monotonicity result still holds whenever $\alpha(t)$ is non-increasing. This section is based on techniques of Perelman [31, Section 1] for the Ricci flow.

In the fourth section, we prove short-time existence for the flow using again a method from Ricci flow theory known as DeTurck's trick (cf. [10]), i.e., we transform the weakly 
parabolic system $(R H)_{\alpha}$ into a strictly parabolic one by pushing it forward with a family of diffeomorphisms. Moreover, we compute the evolution equations for the Ricci and scalar curvature, the gradient of $\phi$ and combinations thereof. In particular, the evolution equations for the symmetric tensor $S_{i j}:=R_{i j}-\alpha \nabla_{i} \phi \nabla_{j} \phi$ and its trace $S=R-\alpha|\nabla \phi|^{2}$ will be very useful.

In Section 5, we study first consequences of the evolution equations for the existence or non-existence of certain types of singularities. Using the maximum principle, we show that $\min _{x \in M} S(x, t)$ is non-decreasing along the flow. This has the rather surprising consequence that if $|\nabla \phi|^{2}\left(x_{k}, t_{k}\right) \rightarrow \infty$ for $t_{k} \nearrow T$, then $R\left(x_{k}, t_{k}\right)$ blows up as well, i.e., $g\left(t_{k}\right)$ must become singular as $t_{k} \nearrow T$. Conversely, if $|\mathrm{Rm}|$ stays bounded along the flow, $|\nabla \phi|^{2}$ must stay bounded, too. This leads to the conjecture that a uniform Riemann-bound is enough to conclude long-time existence. This conjecture is proved in Section 6. To this end, we first compute estimates for the Riemannian curvature tensor, its derivatives and the higher derivatives of $\phi$ and then follow Bando's [1] and Shi's [38] results for the Ricci flow to derive interior-in-time gradient estimates.

In Section 7, we introduce an entropy functional $W_{\alpha}(g, \phi, f, \tau)$ which corresponds to Perelman's shrinker entropy for the Ricci flow [31, Section 3]. Here $\tau=T-t$ denotes a backwards time. For $\alpha(t) \equiv \alpha>0$, the entropy functional is non-decreasing and constant exactly on shrinking solitons. Again, the entropy is monotone if we allow non-increasing positive coupling functions $\alpha(t)$ instead of constant ones. Using $\mathcal{F}_{\alpha}$ and $\mathcal{W}_{\alpha}$ we can exclude nontrivial breathers, i.e., we show that a breather has to be a gradient soliton. In the case of a steady or expanding breather the result is even stronger, namely we can show that $\phi(t)$ has to be harmonic in these cases for all $t$.

Finally in the last section, we state the monotonicity of a backwards reduced volume quantity for the $(R H)_{\alpha}$ flow with positive non-increasing $\alpha(t)$. This follows from our more general result from [29]. We apply this monotonicity to deduce a local non-collapsing theorem.

In the appendix, we collect the commutator identities on bundles like $T^{*} M \otimes \phi^{*} T N$, which we need for the evolution equations in Sections 4 and 6.

This article originates from the author's PhD thesis [28] from 2009, where some of the proofs and computations are carried out in more details. The author likes to thank Klaus Ecker, Robert Haslhofer, Gerhard Huisken, Tom Ilmanen, Peter Topping and in particular Michael Struwe for stimulating discussions and valuable remarks and suggestions while studying this new flow. Moreover, he thanks the Swiss National Science Foundation that partially supported his research and Zindine Djadli who translated the abstract into flawless French.

\section{Examples and special solutions}

In this section, we only consider time-independent coupling constants $\alpha(t) \equiv \alpha$. First, we study two very simple homogeneous examples for the $(R H)_{\alpha}$ flow system to illustrate the different behavior of the flow for different coupling constants $\alpha$. In particular, the existence or non-existence of singularities will depend on the choice of $\alpha$. We study the volume-preserving 
version of the flow as well. We say that $(g(t), \phi(t))$ is a solution of the normalized $(R H)_{\alpha}$ flow, if it satisfies

$$
\left\{\begin{array}{l}
\frac{\partial}{\partial t} g=-2 \mathrm{Rc}+2 \alpha \nabla \phi \otimes \nabla \phi+\frac{2}{m} g f_{M}\left(R-\alpha|\nabla \phi|^{2}\right) d V \\
\frac{\partial}{\partial t} \phi=\tau_{g} \phi
\end{array}\right.
$$

\subsection{Two homogeneous examples with $\phi=$ id}

Assume that $(M, g(0))$ is a round two-sphere of constant Gauss curvature 1. Under the Ricci flow, the sphere shrinks to a point in finite time. Let us now consider the $(R H)_{\alpha}$ flow, assuming that $(N, \gamma)=(M, g(0))$ and $\phi(0)$ is the identity map. With the ansatz $g(t)=c(t) g(0), c(0)=1$ and the fact that $\phi(t)=\phi(0)$ is harmonic for all $g(t)$, the $(R H)_{\alpha}$ flow reduces to

$$
\frac{\partial}{\partial t} c(t)=-2+2 \alpha
$$

For $\alpha<1, c(t)$ goes to zero in finite time, i.e., $(M, g(t))$ shrinks to a point, while the scalar curvature $R$ and the energy density $|\nabla \phi|^{2}$ both go to infinity. For $\alpha=1$, the solution is stationary. For $\alpha>1, c(t)$ grows linearly and the flow exists forever, while both the scalar curvature $R$ and the energy density $|\nabla \phi|^{2}$ vanish asymptotically. Instead of changing $\alpha$, we can also scale the metric $\gamma$ on the target manifold. Mapping into a larger sphere has the same consequences as choosing a larger $\alpha$. Note that the volume-preserving version (2.1) of the flow is always stationary, as it is for the normalized Ricci flow, too.

A more interesting example is obtained if we let $\left(M^{4}, g(t)\right)=\left(\mathbb{S}^{2} \times L, c(t) g_{\mathbb{S}^{2}} \oplus d(t) g_{L}\right)$, where $\left(\mathbb{S}^{2}, g_{\mathbb{S}^{2}}\right)$ is again a round sphere with Gauss curvature 1 and $\left(L, g_{L}\right)$ is a surface (of genus $\geq 2$ ) with constant Gauss curvature -1 . Under the Ricci flow, $\frac{\partial}{\partial t} c(t)=-2$ and $\frac{\partial}{\partial t} d(t)=+2$. In particular, $c(t)$ goes to zero in finite time while $d(t)$ always expands. Under the normalized Ricci flow $\frac{\partial}{\partial t} g=-2 \mathrm{Rc}+\frac{1}{2} g f R d V$, we have

$$
\frac{\partial}{\partial t} c=-2+\frac{d-c}{d}=-1-c^{2}, \quad \frac{\partial}{\partial t} d=+2+\frac{d-c}{c}=+1+d^{2} .
$$

Again, $c(t)$ goes to zero in finite time. At the same time, $d(t)$ goes to infinity. Now, let us consider the $(R H)_{\alpha}$ flow for this example, setting $(N, \gamma)=(M, g(0))$ and $\phi(0)=$ id. First, note that $\phi(0)$ is always harmonic and thus $\phi(t)=\phi(0)$ is unchanged. The identity map between the same manifold with two different metrics is not necessarily harmonic in general, but here it is. The flow equations reduce to $\frac{\partial}{\partial t} c(t)=-2+2 \alpha$ and $\frac{\partial}{\partial t} d(t)=+2+2 \alpha$. While $d(t)$ always grows, the behavior of $c(t)$ is exactly the same as in the first example above, where we only had a two-sphere. On the other hand, if we consider the normalized flow (2.1), we obtain

$$
\frac{\partial}{\partial t} c=(\alpha-1)-(\alpha+1) c^{2}, \quad \frac{\partial}{\partial t} d=(\alpha+1)-(\alpha-1) d^{2} .
$$

In the case where $\alpha<1, c(t)$ goes to zero in finite time, while $d(t)$ blows up at the same time, similar to the normalized Ricci flow above. For $\alpha=1, c(t)=(1+2 t)^{-1}$ goes to zero in infinite time while $d(t)=(1+2 t)$ grows linearly, i.e., we have long-time existence but no natural convergence (to a manifold with the same topology). In the third case, where $\alpha>1$, both $c(t)$ and $d(t)$ converge with

$$
c(t) \rightarrow \sqrt{\frac{\alpha-1}{\alpha+1}}, \quad d(t) \rightarrow \sqrt{\frac{\alpha+1}{\alpha-1}}, \quad \text { as } t \rightarrow \infty .
$$


These examples show that both the unnormalized and the normalized version of our flow can behave very differently from the Ricci flow if $\alpha$ is chosen large. In particular, they may be more regular in special situations.

\subsection{Volume-preserving version of $(R H)_{\alpha}$}

Here, we show that the unnormalized $(R H)_{\alpha}$ flow and the normalized version are related by rescaling the metric $g$ and the time, while keeping the map $\phi$ unchanged. Indeed, assume that $(g(t), \phi(t))_{t \in[0, T)}$ is a solution of $(R H)_{\alpha}$. Define a family of rescaling factors $\lambda(t)$ by

$$
\lambda(t):=\left(\int_{M} d V_{g(t)}\right)^{-2 / m}, \quad t \in[0, T),
$$

and let $\bar{g}(t)$ be the family of rescaled metrics $\bar{g}(t)=\lambda(t) g(t)$ having constant unit volume

$$
\int_{M} d V_{\bar{g}(t)}=\int_{M} \lambda^{m / 2}(t) d V_{g(t)}=1, \quad \forall t \in[0, T) .
$$

Write $\phi=\mathrm{Rc}-\alpha \nabla \phi \otimes \nabla \phi$ with trace $S=R-\alpha|\nabla \phi|^{2}$. It is an immediate consequence of the scaling behavior of Rc, $R, \nabla \phi \otimes \nabla \phi$ and $|\nabla \phi|^{2}$ that $\bar{\phi}=\varnothing$ and $\bar{S}=\lambda^{-1} S$ for $\bar{g}=\lambda g$. Note that $\lambda(t)$ is a smooth function of time, with

$$
\frac{d}{d t} \lambda(t)=-\frac{2}{m}\left(\int_{M} d V_{g}\right)^{-\frac{2+m}{m}} \int_{M}(-S) d V_{g}=\frac{2}{m} \lambda f_{M} S d V_{g}=\frac{2}{m} \lambda^{2} f_{M} \bar{S} d V_{\bar{g}} .
$$

Now, we rescale the time. Put $\bar{t}(t):=\int_{0}^{t} \lambda(s) d s$, so that $\frac{d \bar{t}}{d t}=\lambda(t)$. Then, we obtain

$$
\begin{aligned}
& \frac{\partial}{\partial \bar{t}} \bar{g}=\lambda^{-1} \frac{\partial}{\partial t}(\lambda g)=\frac{\partial}{\partial t} g+\left(\lambda^{-2} \frac{\partial}{\partial t} \lambda\right) \bar{g}=-2 \bar{\phi}+\frac{2}{m} \bar{g} f_{M} \bar{S} d V_{\bar{g}}, \\
& \frac{\partial}{\partial t} \phi=\lambda^{-1} \frac{\partial}{\partial t} \phi=\lambda^{-1} \tau_{g} \phi=\tau_{\bar{g}} \phi .
\end{aligned}
$$

This means that $(\bar{g}(\bar{t}), \phi(\bar{t}))$ solves the volume-preserving $(R H)_{\alpha}$ flow $(2.1)$ on $[0, \bar{T})$, where $\bar{T}=\int_{0}^{T} \lambda(s) d s$.

\subsection{Gradient solitons}

A solution to $(R H)_{\alpha}$ which changes under a one-parameter family of diffeomorphisms on $M$ and scaling is called a soliton (or a self-similar solution). These solutions correspond to fixed points modulo diffeomorphisms and scaling. The more general class of periodic solutions modulo diffeomorphisms and scaling, the so-called breathers, will be defined (but also ruled out) in Section 7.

Definition 2.1. - A solution $(g(t), \phi(t))_{t \in[0, T)}$ of $(R H)_{\alpha}$ is called a soliton if there exists a one-parameter family of diffeomorphisms $\psi_{t}: M \rightarrow M$ with $\psi_{0}=\mathrm{id}_{M}$ and a scaling function $c:[0, T) \rightarrow \mathbb{R}_{+}$such that

$$
\left\{\begin{array}{l}
g(t)=c(t) \psi_{t}^{*} g(0) \\
\phi(t)=\psi_{t}^{*} \phi(0)
\end{array}\right.
$$

The cases $\frac{\partial}{\partial t} c=\dot{c}<0, \dot{c}=0$ and $\dot{c}>0$ correspond to shrinking, steady and expanding solitons, respectively. If the diffeomorphisms $\psi_{t}$ are generated by a vector field $X(t)$ that is the gradient of some function $f(t)$ on $M$, then the soliton is called gradient soliton and $f$ is called the potential of the soliton. 
Lemma 2.2. - Let $(g(t), \phi(t))_{t \in[0, T)}$ be a gradient soliton with potential $f$. Then for any $t_{0} \in[0, T)$, this soliton satisfies the coupled elliptic system

$$
\left\{\begin{array}{l}
0=\mathrm{Rc}-\alpha \nabla \phi \otimes \nabla \phi+\operatorname{Hess}(f)+\sigma g, \\
0=\tau_{g} \phi-\langle\nabla \phi, \nabla f\rangle,
\end{array}\right.
$$

for some constant $\sigma\left(t_{0}\right)$. Conversely, given a function $f$ on $M$ and a solution of (2.4) at $t=0$, there exist one-parameter families of constants $c(t)$ and diffeomorphisms $\psi_{t}: M \rightarrow M$ such that defining $(g(t), \phi(t))$ as in (2.3) yields a solution of $(R H)_{\alpha}$. Moreover, $c(t)$ can be chosen linear in $t$.

Proof. - Suppose we have a soliton solution to $(R H)_{\alpha}$. Without loss of generality, $c(0)=1$ and $\psi_{0}=\operatorname{id}_{M}$. Thus, the solution satisfies

$$
\begin{aligned}
-2 \operatorname{Rc}(g(0))+2 \alpha(\nabla \phi \otimes \nabla \phi)(0) & =\left.\frac{\partial}{\partial t} g(t)\right|_{t=0}=\left.\frac{\partial}{\partial t}\left(c(t) \psi_{t}^{*} g(0)\right)\right|_{t=0} \\
& =\dot{c}(0) g(0)+\mathscr{L}_{X(0)} g(0) \\
& =\dot{c}(0) g(0)+2 \operatorname{Hess}(f(\cdot, 0)),
\end{aligned}
$$

where $X(t)$ is the family of vector fields generating $\psi_{t}$. Moreover, we compute

$$
\left(\tau_{g} \phi\right)(0)=\left.\frac{\partial}{\partial t} \phi(t)\right|_{t=0}=\mathscr{L}_{X(0)} \phi(0)=\langle\nabla \phi, \nabla f\rangle .
$$

Together, this proves (2.4) with $\sigma=\frac{1}{2} \dot{c}(0)$ for $t_{0}=0$. Hence, by a time-shifting argument, (2.4) must hold for any $t_{0} \in[0, T)$. One can easily see that $\sigma\left(t_{0}\right)=\dot{c}\left(t_{0}\right) / 2 c\left(t_{0}\right)$.

Conversely, let $(g(0), \phi(0))$ solve (2.4) for some function $f$ on $M$. Define $c(t):=1+2 \sigma t$ and $X(t):=\nabla f / c(t)$. Let $\psi_{t}$ be the diffeomorphisms generated by the family of vector fields $X(t)$ (with $\psi_{0}=\mathrm{id}_{M}$ ) and define $(g(t), \phi(t))$ as in (2.3). For $\sigma<0$ this is possible on the time interval $t \in\left[0, \frac{-1}{2 \sigma}\right)$, in the case $\sigma \geq 0$ it is possible for $t \in[0, \infty)$. Then

$$
\begin{aligned}
\frac{\partial}{\partial t} g(t) & =\dot{c}(t) \psi_{t}^{*}(g(0))+c(t) \psi_{t}^{*}\left(\mathscr{L}_{X(t)} g(0)\right)=\psi_{t}^{*}\left(2 \sigma g(0)+\mathscr{L}_{\nabla f} g(0)\right) \\
& =\psi_{t}^{*}(2 \sigma g(0)+2 \operatorname{Hess}(f))=\psi_{t}^{*}(-2 \operatorname{Rc}(g(0))+2 \alpha(\nabla \phi \otimes \nabla \phi)(0)) \\
& =-2 \operatorname{Rc}(g(t))+2 \alpha(\nabla \phi \otimes \nabla \phi)(t),
\end{aligned}
$$

as well as

$$
\begin{aligned}
\frac{\partial}{\partial t} \phi(t) & =\psi_{t}^{*}\left(\mathscr{L}_{X(t)} \phi(0)\right)=\psi_{t}^{*}\langle\nabla \phi(0), \nabla f / c(t)\rangle=c(t)^{-1} \psi_{t}^{*}\left(\tau_{g(0)} \phi(0)\right) \\
& =c(t)^{-1} \tau_{\psi_{t}^{*} g(0)} \phi(t)=\tau_{g(t)} \phi(t) .
\end{aligned}
$$

This means that $(g(t), \phi(t))$ is a solution of $(R H)_{\alpha}$ and thus a soliton solution.

By Lemma 2.2 and rescaling, we may assume that $c(t)=T-t$ for shrinking solitons (here $T$ is the maximal time of existence for the flow), $c(t)=1$ for steady solitons and $c(t)=t-T$ for expanding solitons (where $T$ defines a birth time). An example for a soliton solution is the very first example from this section, where $(M, g(0))=(N, \gamma)=\left(\mathbb{S}^{2}, g_{\mathbb{S}^{2}}\right)$ and $\phi(0)=$ id. For $\alpha<1$, the soliton is shrinking, for $\alpha=1$ steady and for $\alpha>1$ expanding. Since $\phi_{t}=\mathrm{id}_{M}$ for all $t$ in all three cases, these are gradient solitons with potential $f=0$.

Taking the trace of the first equation in (2.4), we see that a soliton must satisfy

$$
R-\alpha|\nabla \phi|^{2}+\triangle f+\sigma m=0 .
$$


Taking covariant derivatives in (2.4) and using the twice traced second Bianchi identity $\nabla_{j} R_{i j}=\frac{1}{2} \nabla_{i} R$, we obtain (analogous to the corresponding equation for soliton solutions of the Ricci flow)

$$
R-\alpha|\nabla \phi|^{2}+|\nabla f|^{2}+2 \sigma f=\text { const } .
$$

Finally, with $f(\cdot, t)=\psi_{t}^{*}(f(\cdot, 0))$, we get

$$
\frac{\partial}{\partial t} f=\mathscr{L}_{X} f=|\nabla f|^{2} .
$$

Combining this with (2.5), we obtain the evolution equation

$$
\left(\frac{\partial}{\partial t}+\triangle\right) f=|\nabla f|^{2}-R+\alpha|\nabla \phi|^{2}-\sigma m .
$$

For steady solitons, for which the formulas (2.4)-(2.8) hold with $\sigma=0$, Equation (2.8) is equivalent to $u=e^{-f}$ solving the adjoint heat equation

$$
\square^{*} u=-\frac{\partial}{\partial t} u-\triangle u+R u-\alpha|\nabla \phi|^{2} u=0 .
$$

For shrinking solitons, (2.4)-(2.8) hold with $\sigma(t)=-\frac{1}{2}(T-t)^{-1}$ and (2.8) is equivalent to $u=(4 \pi(T-t))^{-m / 2} e^{-f}$ solving the adjoint heat equation. Finally, for expanding solitons, $\sigma(t)=+\frac{1}{2}(t-T)^{-1}$ and (2.8) is equivalent to the fact that $u=(4 \pi(t-T))^{-m / 2} e^{-f}$ solves the adjoint heat equation (2.9).

\section{The $(R H)_{\alpha}$ flow as a gradient flow}

In this section, we introduce an energy functional $\mathcal{F}_{\alpha}$ for the $(R H)_{\alpha}$ flow, which corresponds to Perelman's $\mathcal{F}$-energy for the Ricci flow introduced in [31, Section 1]. For a detailed study of Perelman's functional, we refer to Chow et al. [4, Chapter 5], Müller [27, Chapter 3], or Topping [43, Chapter 6]. In the special case $N \subseteq \mathbb{R}$, the corresponding functional was introduced by List [22]. We follow his work closely in the first part of this section.

\subsection{The energy functional and its first variation}

Let $g=g_{i j} \in \Gamma\left(\operatorname{Sym}_{+}^{2}\left(T^{*} M\right)\right)$ be a Riemannian metric on a closed manifold $M$, $f: M \rightarrow \mathbb{R}$ a smooth function and $\phi \in C^{\infty}(M, N):=\left\{\phi \in C^{\infty}\left(M, \mathbb{R}^{d}\right) \mid \phi(M) \subseteq N\right\}$. For a constant $\alpha(t) \equiv \alpha>0$, we set

$$
\mathcal{F}_{\alpha}(g, \phi, f):=\int_{M}\left(R_{g}+|\nabla f|_{g}^{2}-\alpha|\nabla \phi|_{g}^{2}\right) e^{-f} d V_{g}
$$

Take variations

$$
\begin{aligned}
g_{i j}^{\varepsilon} & =g_{i j}+\varepsilon h_{i j}, & h_{i j} & \in \Gamma\left(\operatorname{Sym}^{2}\left(T^{*} M\right)\right), \\
f^{\varepsilon} & =f+\varepsilon \ell, & \ell & \in C^{\infty}(M), \\
\phi^{\varepsilon} & =\pi_{N}(\phi+\varepsilon \vartheta), & \vartheta & \in C^{\infty}\left(M, \mathbb{R}^{d}\right) \text { with } \vartheta(x) \in T_{\phi(x)} N,
\end{aligned}
$$

where $\pi_{N}$ is the smooth nearest-neighbor projection defined on a tubular neighborhood of $N \subset \mathbb{R}^{d}$. Note that we used the identification $T_{p} N \subset T_{p} \mathbb{R}^{d} \cong \mathbb{R}^{d}$. We denote by $\delta$ the 
derivative $\delta=\left.\frac{d}{d \varepsilon}\right|_{\varepsilon=0}$, i.e., we have $\delta g=h, \delta f=\ell$ and $\delta \phi=\left(d \pi_{N} \circ \phi\right) \vartheta=\vartheta$. Our goal is to compute

$$
\begin{aligned}
\delta \mathcal{F}_{\alpha, g, \phi, f}(h, \vartheta, \ell) & :=\left.\frac{d}{d \varepsilon}\right|_{\varepsilon=0} \mathcal{F}_{\alpha}\left(g+\varepsilon h, \pi_{N}(\phi+\varepsilon \vartheta), f+\varepsilon \ell\right) \\
& =\underbrace{\delta \int_{M}\left(R+|\nabla f|^{2}\right) e^{-f} d V}_{=: I}-\alpha \cdot \underbrace{\delta \int_{M}|\nabla \phi|^{2} e^{-f} d V}_{=: I I} .
\end{aligned}
$$

For the variation of the first integral, we know from the Ricci flow theory that

$$
I=\int_{M}\left(-h^{i j}\left(R_{i j}+\nabla_{i} \nabla_{j} f\right)+\left(\frac{1}{2} \operatorname{tr}_{g} h-\ell\right)\left(2 \triangle f-|\nabla f|^{2}+R\right)\right) e^{-f} d V
$$

see Perelman [31, Section 1], or Müller [27, Lemma 3.3], for a detailed proof. For the variation of the second integral, we compute with a partial integration

$$
\begin{aligned}
I I & =\int_{M} 2 g^{i j} \nabla_{i} \phi^{\lambda} \nabla_{j} \vartheta^{\lambda} e^{-f} d V+\int_{M}\left(-h^{i j} \nabla_{i} \phi^{\lambda} \nabla_{j} \phi^{\lambda}+|\nabla \phi|_{g}^{2}\left(\frac{1}{2} \operatorname{tr}_{g} h-\ell\right)\right) e^{-f} d V, \\
& =\int_{M}\left(-2 \vartheta^{\lambda}\left(\triangle_{g} \phi^{\lambda}-\left\langle\nabla \phi^{\lambda}, \nabla f\right\rangle_{g}\right)-h^{i j} \nabla_{i} \phi^{\lambda} \nabla_{j} \phi^{\lambda}+|\nabla \phi|_{g}^{2}\left(\frac{1}{2} \operatorname{tr}_{g} h-\ell\right)\right) e^{-f} d V .
\end{aligned}
$$

Hence, by putting everything together, we find

$$
\begin{aligned}
\delta \mathcal{F}_{\alpha, g, \phi, f}(h, \vartheta, \ell)= & \int_{M}-h^{i j}\left(R_{i j}+\nabla_{i} \nabla_{j} f-\alpha \nabla_{i} \phi \nabla_{j} \phi\right) e^{-f} d V \\
& +\int_{M}\left(\frac{1}{2} \operatorname{tr}_{g} h-\ell\right)\left(2 \triangle f-|\nabla f|^{2}+R-\alpha|\nabla \phi|^{2}\right) e^{-f} d V \\
& +\int_{M} 2 \alpha \vartheta\left(\tau_{g} \phi-\langle\nabla \phi, \nabla f\rangle\right) e^{-f} d V
\end{aligned}
$$

since $\vartheta \triangle_{g} \phi=\vartheta \tau_{g} \phi$, where $\tau_{g} \phi:=\triangle_{g} \phi-A(\phi)(\nabla \phi, \nabla \phi)_{M}$ denotes the tension field of $\phi$.

\subsection{Gradient flow for fixed background measure}

Now, we fix the measure $d \mu=e^{-f} d V$, i.e., let $f=-\log \left(\frac{d \mu}{d V}\right)$, where $\frac{d \mu}{d V}$ denotes the Radon-Nikodym differential of measures. Then, from $0=\delta d \mu=\left(\frac{1}{2} \operatorname{tr}_{g} h-\ell\right) d \mu$ we get $\ell=\frac{1}{2} \operatorname{tr}_{g} h$. Thus, for a fixed measure $\mu$ the functional $\mathcal{F}_{\alpha}$ and its variation $\delta \mathcal{F}_{\alpha}$ depend only on $g$ and $\phi$ and their variations $\delta g=h$ and $\delta \phi=\vartheta$. In the following we write

$$
\mathcal{F}_{\alpha}^{\mu}(g, \phi):=\mathcal{F}_{\alpha}\left(g, \phi,-\log \left(\frac{d \mu}{d V}\right)\right)
$$

and

$$
\delta \mathcal{F}_{\alpha, g, \phi}^{\mu}(h, \vartheta):=\delta \mathcal{F}_{\alpha, g, \phi,-\log \left(\frac{d \mu}{d V}\right)}\left(h, \vartheta, \frac{1}{2} \operatorname{tr}_{g} h\right) .
$$

Equation (3.2) reduces to

(3.4) $\delta \mathcal{F}_{\alpha, g, \phi}^{\mu}(h, \vartheta)=\int_{M}\left(-h^{i j}\left(R_{i j}+\nabla_{i} \nabla_{j} f-\alpha \nabla_{i} \phi \nabla_{j} \phi\right)+2 \alpha \vartheta\left(\tau_{g} \phi-\langle\nabla \phi, \nabla f\rangle\right)\right) d \mu$.

Let $(g, \phi) \in \Gamma\left(\operatorname{Sym}_{+}^{2}\left(T^{*} M\right)\right) \times C^{\infty}(M, N)$ and define on

$$
H:=H_{g, \phi}=\Gamma\left(\operatorname{Sym}^{2}\left(T^{*} M\right)\right) \times T_{\phi} C^{\infty}(M, N)
$$


an inner product depending on $\alpha$ and the measure $\mu$ by

$$
\left\langle\left(k_{i j}, \psi\right),\left(h_{i j}, \vartheta\right)\right\rangle_{H, \alpha, \mu}:=\int_{M}\left(\frac{1}{2} h^{i j} k_{i j}+2 \alpha \psi \vartheta\right) d \mu .
$$

From $\delta \mathcal{F}_{\alpha, g, \phi}^{\mu}(h, \vartheta)=\left\langle\operatorname{grad} \mathcal{F}_{\alpha}^{\mu}(g, \phi),(h, \vartheta)\right\rangle_{H, \alpha, \mu}$ we then deduce

$$
\operatorname{grad} \mathcal{F}_{\alpha}^{\mu}(g, \phi)=\left(-2\left(R_{i j}+\nabla_{i} \nabla_{j} f-\alpha \nabla_{i} \phi \nabla_{j} \phi\right), \tau_{g} \phi-\langle\nabla \phi, \nabla f\rangle\right) .
$$

Let $\pi_{1}, \pi_{2}$ denote the natural projections of $H$ onto its first and second factors, respectively. Then, the gradient flow of $\mathcal{F}_{\alpha}^{\mu}$ is

$$
\left\{\begin{aligned}
\frac{\partial}{\partial t} g_{i j} & =\pi_{1}\left(\operatorname{grad} \mathcal{F}_{\alpha}^{\mu}(g, \phi)\right), \\
\frac{\partial}{\partial t} \phi & =\pi_{2}\left(\operatorname{grad} \mathcal{F}_{\alpha}^{\mu}(g, \phi)\right) .
\end{aligned}\right.
$$

Thus, recalling the equation $\frac{\partial}{\partial t} f=\ell=\frac{1}{2} \operatorname{tr}_{g}\left(\frac{\partial}{\partial t} g_{i j}\right)$, we obtain the gradient flow system

$$
\left\{\begin{aligned}
\frac{\partial}{\partial t} g_{i j} & =-2\left(R_{i j}+\nabla_{i} \nabla_{j} f-\alpha \nabla_{i} \phi \nabla_{j} \phi\right), \\
\frac{\partial}{\partial t} \phi & =\tau_{g} \phi-\langle\nabla \phi, \nabla f\rangle, \\
\frac{\partial}{\partial t} f & =-R-\triangle f+\alpha|\nabla \phi|^{2} .
\end{aligned}\right.
$$

\subsection{Pulling back with diffeomorphisms}

As one can do for the Ricci flow (see Perelman [31, Section 1] or Müller [27, page 52]), we now pull back a solution $(g, \phi, f)$ of (3.6) with a family of diffeomorphisms generated by $X=\nabla f$. Indeed, recalling the formulas for the Lie derivatives $\left(\mathcal{L}_{\nabla f} g\right)_{i j}=2 \nabla_{i} \nabla_{j} f$, $\mathscr{L}_{\nabla f} \phi=\langle\nabla \phi, \nabla f\rangle$ and $\mathscr{L}_{\nabla f} f=|\nabla f|^{2}$, we can rewrite (3.6) in the form

$$
\left\{\begin{array}{l}
\frac{\partial}{\partial t} g=-2 \mathrm{Rc}+2 \alpha \nabla \phi \otimes \nabla \phi-\left(\mathscr{L}_{\nabla f} g\right), \\
\frac{\partial}{\partial t} \phi=\tau_{g} \phi-\left(\mathscr{L}_{\nabla f} \phi\right), \\
\frac{\partial}{\partial t} f=-\triangle f+|\nabla f|^{2}-R+\alpha|\nabla \phi|^{2}-\left(\mathscr{L}_{\nabla f} f\right) .
\end{array}\right.
$$

Hence, if $\psi_{t}$ is the one-parameter family of diffeomorphisms induced by the vector field $X(t)=\nabla f(t) \in \Gamma(T M), t \in[0, T)$, i.e., if $\frac{\partial}{\partial t} \psi_{t}=X(t) \circ \psi_{t}, \psi_{0}=$ id, then the pulledback quantities $\tilde{g}=\psi_{t}^{*} g, \tilde{\phi}=\psi_{t}^{*} \phi, \tilde{f}=\psi_{t}^{*} f$ satisfy

$$
\left\{\begin{array}{l}
\frac{\partial}{\partial t} \tilde{g}=-2 \tilde{R c}+2 \alpha \nabla \tilde{\phi} \otimes \nabla \tilde{\phi} \\
\frac{\partial}{\partial t} \tilde{\phi}=\tau_{\tilde{g}} \tilde{\phi} \\
\frac{\partial}{\partial t} \tilde{f}=-\triangle_{\tilde{g}} \tilde{f}+|\nabla \tilde{f}|_{\tilde{g}}^{2}-\tilde{R}+\alpha|\nabla \tilde{\phi}|_{\tilde{g}}^{2} .
\end{array}\right.
$$

Here, $\tilde{R c}$ and $\tilde{R}$ denote the Ricci and scalar curvature of $\tilde{g}$ and $\triangle, \tau$ and the norms are also computed with respect to $\tilde{g}$. In the following, we will usually consider the pulled-back gradient flow system and therefore drop the tildes for convenience of notation.

Note that the formal adjoint of the heat operator $\square=\frac{\partial}{\partial t}-\triangle$ under the flow $\frac{\partial}{\partial t} g=h$ is $\square^{*}=-\frac{\partial}{\partial t}-\triangle-\frac{1}{2} \operatorname{tr}_{g} h$. Indeed, for functions $v, w: M \times[0, T] \rightarrow \mathbb{R}$, a straightforward computation yields

$$
\int_{0}^{T} \int_{M}(\square v) w d V d t=\left[\int_{M} v w d V\right]_{0}^{T}+\int_{0}^{T} \int_{M} v\left(\square^{*} w\right) d V d t .
$$

$4^{\mathrm{e}}$ SÉRIE - TOME 45 - 2012 - No 1 
In our case where $h_{i j}=-2 R_{i j}+2 \alpha \nabla_{i} \phi \nabla_{j} \phi$, this is $\square^{*}=-\frac{\partial}{\partial t}-\triangle+R-\alpha|\nabla \phi|^{2}$ and thus the evolution equation for $f$ is equivalent to $e^{-f}$ solving the adjoint heat equation $\square^{*} e^{-f}=0$. The system now reads

$$
\left\{\begin{aligned}
\frac{\partial}{\partial t} g & =-2 \mathrm{Rc}+2 \alpha \nabla \phi \otimes \nabla \phi, \\
\frac{\partial}{\partial t} \phi & =\tau_{g} \phi, \\
0 & =\square^{*} e^{-f} .
\end{aligned}\right.
$$

This means that $(R H)_{\alpha}$ can be interpreted as the gradient flow of $\mathcal{F}_{\alpha}^{\mu}$ for any fixed background measure $\mu$. Moreover, using (3.4), (3.5) and the diffeomorphism invariance of $\mathcal{F}_{\alpha}$, we get the following.

Proposition 3.1. - Let $(g(t), \phi(t))_{t \in[0, T)}$ be a solution of the $(R H)_{\alpha}$ flow with coupling constant $\alpha(t) \equiv \alpha>0$ and let $e^{-f}$ solve the adjoint heat equation under this flow. Then the energy functional $\mathcal{F}_{\alpha}(g, \phi, f)$ defined in (3.1) is non-decreasing with

$$
\frac{d}{d t} \mathcal{F}_{\alpha}=\int_{M}\left(2|\operatorname{Rc}-\alpha \nabla \phi \otimes \nabla \phi+\operatorname{Hess}(f)|^{2}+2 \alpha\left|\tau_{g} \phi-\langle\nabla \phi, \nabla f\rangle\right|^{2}\right) e^{-f} d V \geq 0
$$

Moreover, $\mathcal{F}_{\alpha}$ is constant if and only if $(g(t), \phi(t))$ is a steady soliton.

Allowing also time-dependent coupling constants $\alpha(t)$, we obtain the following.

Corollary 3.2. - Let $(g(t), \phi(t))_{t \in[0, T)}$ solve $(R H)_{\alpha}$ for a positive coupling function $\alpha(t)$ and let $e^{-f}$ solve the adjoint heat equation under this flow. Then $\mathcal{F}_{\alpha(t)}(g(t), \phi(t), f(t))$ satisfies

$$
\frac{d}{d t} \mathcal{F}_{\alpha}=\int_{M}\left(2|\operatorname{Rc}-\alpha \nabla \phi \otimes \nabla \phi+\operatorname{Hess}(f)|^{2}+2 \alpha\left|\tau_{g} \phi-\langle\nabla \phi, \nabla f\rangle\right|^{2}-\dot{\alpha}|\nabla \phi|^{2}\right) e^{-f} d V,
$$

in particular, it is non-decreasing if $\alpha(t)$ is a non-increasing function.

\subsection{Minimizing over all probability measures}

Following Perelman [31], we define

$$
\lambda_{\alpha}(g, \phi):=\inf \left\{\mathcal{F}_{\alpha}^{\mu}(g, \phi) \mid \mu(M)=1\right\}=\inf \left\{\mathcal{F}_{\alpha}(g, \phi, f) \mid \int_{M} e^{-f} d V=1\right\} .
$$

The first task is to show that the infimum is always achieved. Indeed, if we set $v=e^{-f / 2}$, we can write the energy as

$$
\mathcal{F}_{\alpha}(g, \phi, v)=\int_{M}\left(R v^{2}+4|\nabla v|^{2}-\alpha|\nabla \phi|^{2} v^{2}\right) d V=\int_{M} v\left(R v-4 \triangle v-\alpha|\nabla \phi|^{2} v\right) d V .
$$

Hence

$$
\lambda_{\alpha}(g, \phi)=\inf \left\{\int_{M} v\left(R v-4 \triangle v-\alpha|\nabla \phi|^{2} v\right) d V \mid \int_{M} v^{2} d V=1\right\}
$$

is the smallest eigenvalue of the operator $-4 \triangle+R-\alpha|\nabla \phi|^{2}$ and $v$ is a corresponding normalized eigenvector. Since the operator (for any time $t$ and map $\phi(t)$ ) is a Schrödinger operator, there exists a unique positive and normalized eigenvector $v_{\min }(t)$, see for example Reed and Simon [35] and Rothaus [36]. From eigenvalue perturbation theory, we see that if $g(t)$ and $\phi(t)$ depend smoothly on $t$, then so do $\lambda_{\alpha}(g(t), \phi(t))$ and $v_{\min }(t)$. 
Proposition 3.3. - Let $(g(t), \phi(t))_{t \in[0, T)}$ be a smooth solution of the $(R H)_{\alpha}$ flow with constant $\alpha(t) \equiv \alpha>0$. Then $\lambda_{\alpha}(g, \phi)$ as defined in (3.9) is monotone non-decreasing in time and it is constant if and only if

$$
\left\{\begin{array}{l}
0=\mathrm{Rc}-\alpha \nabla \phi \otimes \nabla \phi+\operatorname{Hess}(f), \\
0=\tau_{g} \phi-\langle\nabla \phi, \nabla f\rangle,
\end{array}\right.
$$

for the minimizing function $f=-2 \log v_{\min }$.

Proof. - Pick arbitrary times $t_{1}, t_{2} \in[0, T)$ and let $v_{\min }\left(t_{2}\right)$ be the unique positive minimizer for $\lambda_{\alpha}\left(g\left(t_{2}\right), \phi\left(t_{2}\right)\right)$. Put $u\left(t_{2}\right)=v_{\min }^{2}\left(t_{2}\right)>0$ and solve the adjoint heat equation $\square^{*} u=0$ backwards on $\left[t_{1}, t_{2}\right]$. Note that $u\left(x^{\prime}, t^{\prime}\right)>0$ for all $x^{\prime} \in M$ and $t^{\prime} \in\left[t_{1}, t_{2}\right]$ by the maximum principle and the constraint $\int_{M} u d V=\int_{M} v^{2} d V=1$ is preserved since

$$
\frac{d}{d t} \int_{M} u d V=\int_{M}\left(\frac{\partial}{\partial t} u\right) d V+\int_{M} u\left(\frac{\partial}{\partial t} d V\right)=-\int_{M} \triangle u d V=0 .
$$

Here, we used $\frac{\partial}{\partial t} d V=-\frac{1}{2} \operatorname{tr}_{g}\left(\frac{\partial}{\partial t} g\right) d V=\left(-R+\alpha|\nabla \phi|^{2}\right) d V$ and $\frac{\partial}{\partial t} u=\left(-\triangle+R-\alpha|\nabla \phi|^{2}\right) u$, the latter following from $\square^{*} u=0$. Thus, with $u(t)=e^{-\bar{f}(t)}$ for all $t \in\left[t_{1}, t_{2}\right]$, we obtain with Proposition 3.1

$$
\lambda_{\alpha}\left(g\left(t_{1}\right), \phi\left(t_{1}\right)\right) \leq \mathcal{F}_{\alpha}\left(g\left(t_{1}\right), \phi\left(t_{1}\right), \bar{f}\left(t_{1}\right)\right) \leq \mathcal{F}_{\alpha}\left(g\left(t_{2}\right), \phi\left(t_{2}\right), \bar{f}\left(t_{2}\right)\right)=\lambda_{\alpha}\left(g\left(t_{2}\right), \phi\left(t_{2}\right)\right) .
$$

The condition (3.10) in the equality case follows directly from (3.8).

Again the monotonicity of $\lambda_{\alpha}(g, \phi)$ is preserved if we allow a positive non-increasing coupling function $\alpha(t)$ instead of a time-independent positive constant $\alpha$.

\section{Short-time existence and evolution equations}

Due to diffeomorphism invariance, our flow is only weakly parabolic. In fact, the principal symbol for the first equation is the same as for the Ricci flow since the additional term is of lower order. Thus, one cannot directly apply the standard parabolic existence theory. Fortunately, shortly after Hamilton's first proof of short-time existence for the Ricci flow in [15] which was based on the Nash-Moser implicit function theorem, DeTurck [10] found a substantially simpler proof which can easily be modified to get an existence proof for our system $(R H)_{\alpha}$. Note that we only consider the case where $M$ is closed, but following Shi's short-time existence proof in [38] for the Ricci flow on complete noncompact manifolds, one can also prove more general short-time existence results for our flow. We first recall some results for the Ricci flow, following the presentation of Hamilton [16] very closely.

Since some results strongly depend on the curvature of $(N, \gamma)$, it is more convenient to work with $\phi: M \rightarrow N$ itself instead of $e_{N} \circ \phi: M \rightarrow \mathbb{R}^{d}$ as in the last section. Therefore, repeated Greek indices are summed over from 1 to $n=\operatorname{dim} N$ in this section. 


\subsection{Dual Ricci-Harmonic and Ricci-DeTurck flow}

Let $g(t)$ be a solution of the Ricci flow and $\psi(t):(M, g) \rightarrow(M, h)$ a one parameter family of smooth maps satisfying the harmonic map flow $\frac{\partial}{\partial t} \psi=\tau_{g} \psi$ with respect to the evolving metric $g$. Note that this is $(R H)_{\alpha \equiv 0}$. If $\psi(t)$ is a diffeomorphism at time $t=0$, it will stay a diffeomorphism for at least a short time. Now, we consider the push-forward $\tilde{g}:=\psi_{*} g$ of the metric $g$ under $\psi$. The evolution equation for $\tilde{g}$ reads

$$
\frac{\partial}{\partial t} \tilde{g}_{i j}=-2 \tilde{R}_{i j}+\left(\mathcal{L}_{V} \tilde{g}\right)_{i j}=-2 \tilde{R}_{i j}+\tilde{\nabla}_{i} V_{j}+\tilde{\nabla}_{j} V_{i},
$$

where $\tilde{\nabla}$ denotes the Levi-Civita connection of $\tilde{g}$ and $\frac{\partial}{\partial t} \psi=-V \circ \psi$. One calls this the dual Ricci-Harmonic flow or also the $h$-flow. An easy computation (see DeTurck [10] or Chow and Knopf [6, Chapter 3]) shows that $V$ is given by

$$
V^{\ell}=\tilde{g}^{i j}\left({ }^{\tilde{g}} \Gamma_{i j}^{\ell}-{ }^{h} \Gamma_{i j}^{\ell}\right),
$$

the trace of the tensor which is the difference between the Christoffel symbols of the connections of $\tilde{g}$ and of $h$, respectively. Note that the evolution equation of $\tilde{g}$ involves only the metrics $\tilde{g}$ and $h$ and not the metric $g$, and since it involves ${ }^{h} \Gamma$ for the fixed background metric $h$ it is no longer diffeomorphism invariant. Indeed, one can show (see Hamilton [16, Section 6]) that

$$
\frac{\partial}{\partial t} \tilde{g}_{i j}=\tilde{g}^{k \ell} \tilde{\nabla}_{k} \hat{\nabla}_{\ell} \tilde{g}_{i j}
$$

where $\hat{\nabla}$ denotes the connection of the background metric $h$. Since $\hat{\nabla}$ is independent of $\tilde{g}$ and $\tilde{\nabla}$ only involves first derivatives of $\tilde{g}$ this is a quasilinear equation. Its principal symbol is $\sigma(\xi)=\tilde{g}^{i j} \xi_{i} \xi_{j} \cdot$ id, where id is the identity on tensors $\tilde{g}$. Hence this flow equation is strictly parabolic and we get short-time existence from the standard parabolic theory for quasilinear equations, see e.g. [24] for a recent and detailed proof. If we additionally assume that $(M, h)=\left(M, g_{0}\right)$ and $\psi(0)=\operatorname{id}_{M}$, the flow $\tilde{g}$ which has the same initial data $\tilde{g}(0)=g_{0}$ as $g$ is called the Ricci-DeTurck flow [10].

Now, one can find a solution to the Ricci flow with smooth initial metric $g(0)=g_{0}$ as follows. Choose any diffeomorphism $\psi(0): M \rightarrow M$. Since $g(0)$ is smooth, its push-forward $\tilde{g}(0)$ is also smooth and Equation (4.3) has a smooth solution for a short time. Next, one computes the vector field $V$ with (4.2) and solves the ODE system

$$
\frac{\partial}{\partial t} \psi=-V \circ \psi
$$

One then recovers $g$ as the pull-back $g=\psi^{*} \tilde{g}$. This method also proves uniqueness of the Ricci flow. Indeed, let $g^{1}(t)$ and $g^{2}(t)$ be two solutions of the Ricci flow equation for $t \in[0, T)$ satisfying $g^{1}(0)=g^{2}(0)$. Then one can solve the harmonic map heat flows $\frac{\partial}{\partial t} \psi^{i}=\tau_{g^{i}} \psi^{i}$, $i \in\{1,2\}$ with $\psi^{1}(0)=\psi^{2}(0)$. This yields two solutions $\tilde{g}^{i}=\psi_{*}^{i} g^{i}$ of the dual RicciHarmonic map flow with the same initial values, hence they must agree. Then the corresponding vector fields $V^{i}$ agree and the two ODE systems $\frac{\partial}{\partial t} \psi^{i}=-V^{i} \circ \psi^{i}$ with the same initial data must have the same solutions $\psi^{1} \equiv \psi^{2}$. Hence also the pull-back metrics $g^{1}$ and $g^{2}$ must agree for all $t \in[0, T)$. 
For the dual Ricci-Harmonic flow, the evolution equations in coordinate form, using only the fixed connection $\hat{\nabla}$ of the background metric $h$, are (see e.g. Simon [39])

$$
\begin{aligned}
\frac{\partial}{\partial t} \tilde{g}_{i j}= & \tilde{g}^{k \ell} \hat{\nabla}_{k} \hat{\nabla}_{\ell} \tilde{g}_{i j}-\tilde{g}^{k \ell} \tilde{g}_{i p} h^{p q} \hat{R}_{j k q \ell}-\tilde{g}^{k \ell} \tilde{g}_{j p} h^{p q} \hat{R}_{i k q \ell} \\
& +\frac{1}{2} \tilde{g}^{k \ell} \tilde{g}^{p q}\left(\hat{\nabla}_{i} \tilde{g}_{p k} \hat{\nabla}_{j} \tilde{g}_{q \ell}+2 \hat{\nabla}_{k} \tilde{g}_{i p} \hat{\nabla}_{q} \tilde{g}_{j \ell}-2 \hat{\nabla}_{k} \tilde{g}_{i p} \hat{\nabla}_{\ell} \tilde{g}_{j q}-4 \hat{\nabla}_{i} \tilde{g}_{p k} \hat{\nabla}_{\ell} \tilde{g}_{j q}\right),
\end{aligned}
$$

where $\hat{R}_{i j k l}=(\operatorname{Rm}(h))_{i j k l}$ denotes the Riemannian curvature tensor of $h$.

Recent work of Isenberg, Guenther and Knopf [14], Schnürer, Schulze and Simon [37] and others shows that DeTurck's trick is not only useful to prove short-time existence for the Ricci flow, but is also useful for convergence and stability results. Their results show that the $h$-flow itself is also interesting to study. In this article however, we only use it as a technical tool.

\subsection{Short-time existence and uniqueness for $(R H)_{\alpha}$}

Let $(g(t), \phi(t))_{t \in[0, T)}$ be a solution of the $(R H)_{\alpha}$ flow with initial data $(g(0), \phi(0))=$ $\left(g_{0}, \phi_{0}\right)$. As for the Ricci-DeTurck flow above, we now let $\psi(t):(M, g(t)) \rightarrow\left(M, g_{0}\right)$ be a solution of the harmonic map heat flow $\frac{\partial}{\partial t} \psi=\tau_{g} \psi$ with $\psi(0)=\operatorname{id}_{M}$ and denote by $(\tilde{g}(t), \tilde{\phi}(t))$ the push-forward of $(g(t), \phi(t))$ with $\psi$. Analogous to Formula (4.1) above, we find

$$
\begin{aligned}
\frac{\partial}{\partial t} \tilde{g}_{i j} & =\psi_{*}\left(\frac{\partial}{\partial t} g\right)_{i j}+\left(\mathscr{L}_{V} \tilde{g}\right)_{i j}=-2 \tilde{R}_{i j}+2 \alpha \nabla_{i} \tilde{\phi} \nabla_{j} \tilde{\phi}+\tilde{\nabla}_{i} V_{j}+\tilde{\nabla}_{j} V_{i}, \\
\frac{\partial}{\partial t} \tilde{\phi} & =\psi_{*}\left(\frac{\partial}{\partial t} \phi\right)+\mathscr{L}_{V} \tilde{\phi}=\tau_{\tilde{g}} \tilde{\phi}+\langle\nabla \tilde{\phi}, V\rangle,
\end{aligned}
$$

where $V^{\ell}=\tilde{g}^{i j}\left({ }^{\tilde{g}} \Gamma_{i j}^{\ell}-{ }^{g_{0}} \Gamma_{i j}^{\ell}\right)$ and $\tilde{\nabla}$ denotes the covariant derivative with respect to $\tilde{g}$. Note that $\nabla_{i} \tilde{\phi} \nabla_{j} \tilde{\phi}=(d \tilde{\phi} \otimes d \tilde{\phi})_{i j}$ as well as $\langle\nabla \tilde{\phi}, V\rangle=d \tilde{\phi}(V)$ are independent of the choice of the metric. Using (4.3), we find

$$
\frac{\partial}{\partial t} \tilde{g}_{i j}=\tilde{g}^{k \ell} \tilde{\nabla}_{k} \hat{\nabla}_{\ell} \tilde{g}_{i j}+2 \alpha(\nabla \tilde{\phi} \otimes \nabla \tilde{\phi})_{i j},
$$

which is again quasilinear strictly parabolic. The explicit evolution equation involving only the fixed Levi-Civita connection $\hat{\nabla}$ of $g_{0}$ can be found from (4.4) by adding $2 \alpha \hat{\nabla}_{i} \tilde{\phi} \hat{\nabla}_{j} \tilde{\phi}$ on the right and replacing $h$ by $g_{0}$.

The evolution equation for $\tilde{\phi}(t)$ in terms of $\hat{\nabla}$ can be computed as follows. Using normal coordinates on $(N, \gamma)$ we have ${ }^{N} \Gamma_{\mu \nu}^{\lambda}=0$ at the base point and thus $\tau_{\tilde{g}} \tilde{\phi}=\triangle_{\tilde{g}} \tilde{\phi}$. We find

$$
\begin{aligned}
\frac{\partial}{\partial t} \tilde{\phi}^{\lambda} & =\triangle_{\tilde{g}} \tilde{\phi}^{\lambda}+\left\langle\nabla \tilde{\phi}^{\lambda}, V\right\rangle=\tilde{g}^{k \ell}\left(\partial_{k} \partial_{\ell} \tilde{\phi}^{\lambda}-{ }^{\tilde{g}} \Gamma_{k \ell}^{j} \nabla_{j} \tilde{\phi}^{\lambda}\right)+\nabla_{j} \tilde{\phi}^{\lambda} V^{j} \\
& =\tilde{g}^{k \ell}\left(\hat{\nabla}_{k} \hat{\nabla}_{\ell} \tilde{\phi}^{\lambda}+{ }^{g_{0}} \Gamma_{k \ell}^{j} \nabla_{j} \tilde{\phi}^{\lambda}-{ }^{\tilde{g}} \Gamma_{k \ell}^{j} \nabla_{j} \tilde{\phi}^{\lambda}\right)+\nabla_{j} \tilde{\phi}^{\lambda} \cdot \tilde{g}^{k \ell}\left({ }^{\tilde{g}} \Gamma_{k \ell}^{j}-{ }^{g_{0}} \Gamma_{k \ell}^{j}\right) \\
& =\tilde{g}^{k \ell} \hat{\nabla}_{k} \hat{\nabla}_{\ell} \tilde{\phi}^{\lambda} .
\end{aligned}
$$

Putting these results together, we have proved the following.

Proposition 4.1. - Let $(g(t), \phi(t))$ be a solution of the $(R H)_{\alpha}$ flow with initial data $(g(0), \phi(0))=\left(g_{0}, \phi_{0}\right)$. Let $\psi(t):(M, g(t)) \rightarrow\left(M, g_{0}\right)$ solve the harmonic map heat flow $\frac{\partial}{\partial t} \psi=\tau_{g} \psi$ with $\psi(0)=\operatorname{id}_{M}$ and let $(\tilde{g}(t), \tilde{\phi}(t))$ denote the push-forward of $(g(t), \phi(t))$ with $\psi$.

$4^{\mathrm{e}}$ SÉRIE - TOME $45-2012-\mathrm{N}^{\circ} 1$ 
Let $\hat{\nabla}$ be the (fixed) Levi-Civita connection with respect to $g_{0}$. Then the dual $(R H)_{\alpha}$ flow $(\tilde{g}(t), \tilde{\phi}(t))$ satisfies

$$
\begin{aligned}
\frac{\partial}{\partial t} \tilde{g}_{i j}= & \tilde{g}^{k \ell} \hat{\nabla}_{k} \hat{\nabla}_{\ell} \tilde{g}_{i j}-\tilde{g}^{k \ell} \tilde{g}_{i p} g_{0}^{p q} \hat{R}_{j k q \ell}-\tilde{g}^{k \ell} \tilde{g}_{j p} g_{0}^{p q} \hat{R}_{i k q \ell}+2 \alpha \hat{\nabla}_{i} \tilde{\phi} \hat{\nabla}_{j} \tilde{\phi} \\
& +\frac{1}{2} \tilde{g}^{k \ell} \tilde{g}^{p q}\left(\hat{\nabla}_{i} \tilde{g}_{p k} \hat{\nabla}_{j} \tilde{g}_{q \ell}+2 \hat{\nabla}_{k} \tilde{g}_{i p} \hat{\nabla}_{q} \tilde{g}_{j \ell}-2 \hat{\nabla}_{k} \tilde{g}_{i p} \hat{\nabla}_{\ell} \tilde{g}_{j q}-4 \hat{\nabla}_{i} \tilde{g}_{p k} \hat{\nabla}_{\ell} \tilde{g}_{j q}\right), \\
\frac{\partial}{\partial t} \tilde{\phi}^{\lambda}= & \tilde{g}^{k \ell} \hat{\nabla}_{k} \hat{\nabla}_{\ell} \tilde{\phi}^{\lambda}+\tilde{g}^{k \ell}\left({ }^{N} \Gamma_{\mu \nu}^{\lambda} \circ \phi\right) \hat{\nabla}_{k} \tilde{\phi}^{\mu} \hat{\nabla}_{\ell} \tilde{\phi}^{\nu} .
\end{aligned}
$$

In particular, the principal symbol for both equations is $\sigma(\xi)=\tilde{g}^{i j} \xi_{i} \xi_{j} \cdot \mathrm{id}$, i.e., the push-forward flow is a solution to a system of strictly parabolic equations.

Short-time existence and uniqueness for the dual flow (and hence also for the $(R H)_{\alpha}$ flow itself) now follow exactly as in the simpler case of the Ricci and the dual Ricci-Harmonic flow described above.

\subsection{Evolution equations for $R, \mathrm{Rc},|\nabla \phi|^{2}$ and $\nabla \phi \otimes \nabla \phi$}

In the following, we often use commutator identities on bundles like $T^{*} M \otimes \phi^{*} T N$. The necessary formulas are collected in the appendix. We denote the Riemannian curvature tensor on $(N, \gamma)$ by ${ }^{N} \mathrm{Rm}$ and let $\mathrm{Rm}, \mathrm{Rc}$ and $R$ be the Riemannian, Ricci and scalar curvature on $(M, g)$. Moreover, we write

$$
\begin{aligned}
\langle\mathrm{Rc}, \nabla \phi \otimes \nabla \phi\rangle & :=R_{i j} \nabla_{i} \phi^{\kappa} \nabla_{j} \phi^{\kappa} \\
\left\langle{ }^{N} \operatorname{Rm}\left(\nabla_{i} \phi, \nabla_{j} \phi\right) \nabla_{j} \phi, \nabla_{i} \phi\right\rangle & :={ }^{N} R_{\kappa \mu \lambda \nu} \nabla_{i} \phi^{\kappa} \nabla_{j} \phi^{\mu} \nabla_{i} \phi^{\lambda} \nabla_{j} \phi^{\nu} .
\end{aligned}
$$

Finally, we use the fact that $\tau_{g} \phi=\nabla_{p} \nabla_{p} \phi$ for the covariant derivative $\nabla$ on $T^{*} M \otimes \phi^{*} T N$, cf. Jost [18, Section 8.1]. From the commutator identities in the appendix, we immediately obtain for $\phi \in C^{\infty}(M, N)$

$$
\begin{aligned}
\triangle_{g}\left(\nabla_{i} \phi \nabla_{j} \phi\right)= & \nabla_{i} \tau_{g} \phi \nabla_{j} \phi+\nabla_{i} \phi \nabla_{j} \tau_{g} \phi+2 \nabla_{i} \nabla_{p} \phi \nabla_{j} \nabla_{p} \phi+R_{i p} \nabla_{p} \phi \nabla_{j} \phi \\
& +R_{j p} \nabla_{p} \phi \nabla_{i} \phi-2\left\langle{ }^{N} \operatorname{Rm}\left(\nabla_{i} \phi, \nabla_{p} \phi\right) \nabla_{p} \phi, \nabla_{j} \phi\right\rangle .
\end{aligned}
$$

REMARK 4.2. - Taking the trace and using $\tau_{g} \phi=0$, we find the well-known Bochner identity for harmonic maps, cf. Jost [18, Section 8.7],

$$
-\triangle_{g}|\nabla \phi|^{2}+2\left|\nabla^{2} \phi\right|^{2}+2\langle\operatorname{Rc}, \nabla \phi \otimes \nabla \phi\rangle=2\left\langle{ }^{N} \operatorname{Rm}\left(\nabla_{i} \phi, \nabla_{j} \phi\right) \nabla_{j} \phi, \nabla_{i} \phi\right\rangle .
$$

Now, we compute the evolution equations for the scalar and Ricci curvature on $M$.

Proposition 4.3. - Let $(g(t), \phi(t))$ be a solution to the $(R H)_{\alpha}$ flow equation. Then the scalar curvature evolves according to

$$
\begin{aligned}
\frac{\partial}{\partial t} R= & \Delta R+2|\mathrm{Rc}|^{2}-4 \alpha\langle\mathrm{Rc}, \nabla \phi \otimes \nabla \phi\rangle+2 \alpha\left|\tau_{g} \phi\right|^{2}-2 \alpha\left|\nabla^{2} \phi\right|^{2} \\
& +2 \alpha\left\langle{ }^{N} \operatorname{Rm}\left(\nabla_{i} \phi, \nabla_{j} \phi\right) \nabla_{j} \phi, \nabla_{i} \phi\right\rangle
\end{aligned}
$$

and the Ricci curvature evolves by

$$
\begin{aligned}
\frac{\partial}{\partial t} R_{i j}= & \triangle_{L} R_{i j}-2 R_{i q} R_{j q}+2 R_{i p j q} R_{p q}+2 \alpha \tau_{g} \phi \nabla_{i} \nabla_{j} \phi-2 \alpha \nabla_{p} \nabla_{i} \phi \nabla_{p} \nabla_{j} \phi \\
& +2 \alpha R_{p i j q} \nabla_{p} \phi \nabla_{q} \phi+2 \alpha\left\langle{ }^{N} \operatorname{Rm}\left(\nabla_{i} \phi, \nabla_{p} \phi\right) \nabla_{p} \phi, \nabla_{j} \phi\right\rangle .
\end{aligned}
$$

Here, $\triangle_{L}$ denotes the Lichnerowicz Laplacian, introduced in [21], which is defined on symmetric two-tensors $t_{i j}$ by

$$
\triangle_{L} t_{i j}:=\triangle t_{i j}+2 R_{i p j q} t_{p q}-R_{i p} t_{p j}-R_{j p} t_{p i} .
$$


Proof. - We know that for $\frac{\partial}{\partial t} g_{i j}=h_{i j}$ the evolution equation for the Ricci tensor is given by

$$
\frac{\partial}{\partial t} R_{i j}=-\frac{1}{2} \triangle_{L} h_{i j}+\frac{1}{2} \nabla_{i} \nabla_{p} h_{p j}+\frac{1}{2} \nabla_{j} \nabla_{p} h_{p i}-\frac{1}{2} \nabla_{i} \nabla_{j}\left(\operatorname{tr}_{g} h\right),
$$

see for example [27, Proposition 1.4] for a proof of this general variation formula. For $h_{i j}=-2 R_{i j}$, we obtain (with the twice contracted second Bianchi identity) $\frac{\partial}{\partial t} R_{i j}=\triangle_{L} R_{i j}$. For $h_{i j}=2 \nabla_{i} \phi \nabla_{j} \phi$, we compute, using (4.8)

$$
\begin{aligned}
\frac{\partial}{\partial t} R_{i j}= & -\triangle_{L}\left(\nabla_{i} \phi \nabla_{j} \phi\right)+\nabla_{i} \nabla_{p}\left(\nabla_{p} \phi \nabla_{j} \phi\right)+\nabla_{j} \nabla_{p}\left(\nabla_{p} \phi \nabla_{i} \phi\right)-\nabla_{i} \nabla_{j}\left(\nabla_{p} \phi \nabla_{p} \phi\right) \\
= & 2 \tau_{g} \phi \nabla_{i} \nabla_{j} \phi-2 \nabla_{i} \nabla_{p} \phi \nabla_{j} \nabla_{p} \phi-2 R_{i p j q} \nabla_{p} \phi \nabla_{q} \phi \\
& +2\left\langle{ }^{N} \operatorname{Rm}\left(\nabla_{i} \phi, \nabla_{p} \phi\right) \nabla_{p} \phi, \nabla_{j} \phi\right\rangle .
\end{aligned}
$$

Linearity then yields (4.10). For the evolution equation for $R$, use

$$
\frac{\partial}{\partial t} R=\frac{\partial}{\partial t}\left(g^{i j} R_{i j}\right)=g^{i j}\left(\frac{\partial}{\partial t} R_{i j}\right)+\left(2 R_{i j}-2 \alpha \nabla_{i} \phi \nabla_{j} \phi\right) R_{i j} .
$$

The desired evolution equation (4.9) follows. An alternative and more detailed proof can be found in the author's thesis [28, Proposition 2.3].

Next, we compute the evolution equations for $|\nabla \phi|^{2}$ and $\nabla \phi \otimes \nabla \phi$.

Proposition 4.4. - Let $(g(t), \phi(t))$ be a solution of $(R H)_{\alpha}$. Then the energy density of $\phi$ satisfies the evolution equation

$$
\frac{\partial}{\partial t}|\nabla \phi|^{2}=\triangle|\nabla \phi|^{2}-2 \alpha|\nabla \phi \otimes \nabla \phi|^{2}-2\left|\nabla^{2} \phi\right|^{2}+2\left\langle{ }^{N} \operatorname{Rm}\left(\nabla_{i} \phi, \nabla_{j} \phi\right) \nabla_{j} \phi, \nabla_{i} \phi\right\rangle .
$$

Furthermore, we have

$$
\begin{aligned}
\frac{\partial}{\partial t}\left(\nabla_{i} \phi \nabla_{j} \phi\right)= & \triangle\left(\nabla_{i} \phi \nabla_{j} \phi\right)-2 \nabla_{p} \nabla_{i} \phi \nabla_{p} \nabla_{j} \phi-R_{i p} \nabla_{p} \phi \nabla_{j} \phi-R_{j p} \nabla_{p} \phi \nabla_{i} \phi \\
& +2\left\langle{ }^{N} \operatorname{Rm}\left(\nabla_{i} \phi, \nabla_{p} \phi\right) \nabla_{p} \phi, \nabla_{j} \phi\right\rangle .
\end{aligned}
$$

Proof. - We start with the second statement. We have

$$
\begin{aligned}
\frac{\partial}{\partial t}\left(\nabla_{i} \phi \nabla_{j} \phi\right) & =\left(\nabla_{t} \nabla_{i} \phi\right) \nabla_{j} \phi+\left(\nabla_{t} \nabla_{j} \phi\right) \nabla_{i} \phi \\
& =\nabla_{i}\left(\frac{\partial}{\partial t} \phi\right) \nabla_{j} \phi+\nabla_{j}\left(\frac{\partial}{\partial t} \phi\right) \nabla_{i} \phi \\
& =\nabla_{i} \tau_{g} \phi \nabla_{j} \phi+\nabla_{j} \tau_{g} \phi \nabla_{i} \phi,
\end{aligned}
$$

where the meaning of the covariant time derivative $\nabla_{t}$ is explained in the appendix. The desired evolution equation (4.13) now follows directly from (4.8). We obtain (4.12) from (4.13) by taking the trace,

$$
\begin{aligned}
\frac{\partial}{\partial t}|\nabla \phi|^{2} & =\left(2 R_{i j}-2 \alpha \nabla_{i} \phi \nabla_{j} \phi\right) \nabla_{i} \phi \nabla_{j} \phi+g^{i j} \frac{\partial}{\partial t}\left(\nabla_{i} \phi \nabla_{j} \phi\right) \\
& =-2 \alpha|\nabla \phi \otimes \nabla \phi|^{2}+\triangle|\nabla \phi|^{2}-2\left|\nabla^{2} \phi\right|^{2}+2\left\langle{ }^{N} \operatorname{Rm}\left(\nabla_{i} \phi, \nabla_{j} \phi\right) \nabla_{j} \phi, \nabla_{i} \phi\right\rangle .
\end{aligned}
$$

$4^{\mathrm{e}}$ SÉRIE - TOME $45-2012-\mathrm{N}^{\circ} 1$ 


\subsection{Evolution of $\phi=\mathrm{Rc}-\alpha \nabla \phi \otimes \nabla \phi$ and its trace}

We write again $\phi:=\mathrm{Rc}-\alpha \nabla \phi \otimes \nabla \phi$ with components $S_{i j}=R_{i j}-\alpha \nabla_{i} \phi \nabla_{j} \phi$ and let $S=R-\alpha|\nabla \phi|^{2}$ be its trace. Then, we can write the $(R H)_{\alpha}$ flow as

$$
\left\{\begin{aligned}
\frac{\partial}{\partial t} g_{i j} & =-2 S_{i j}, \\
\frac{\partial}{\partial t} \phi & =\tau_{g} \phi,
\end{aligned}\right.
$$

and the energy from Section 3 as $\mathcal{F}_{\alpha}(g, \phi, f):=\int_{M}\left(S+|\nabla f|_{g}^{2}\right) e^{-f} d V_{g}$. It is thus convenient to study the evolution equations for $\&$ and $S$. Indeed, many terms cancel and we get much nicer equations than in the previous subsection.

Theorem 4.5. - Let $(g(t), \phi(t))$ solve $(R H)_{\alpha}$ with $\alpha(t) \equiv \alpha>0$. Then $\&$ and $S$ defined as above satisfy the following evolution equations

$$
\begin{aligned}
\frac{\partial}{\partial t} S & =\triangle S+2\left|S_{i j}\right|^{2}+2 \alpha\left|\tau_{g} \phi\right|^{2}, \\
\frac{\partial}{\partial t} S_{i j} & =\triangle_{L} S_{i j}+2 \alpha \tau_{g} \phi \nabla_{i} \nabla_{j} \phi .
\end{aligned}
$$

Proof. - This follows directly by combining the evolution equations from Proposition 4.3 with those from Proposition 4.4.

Remark 4.6. - Note that in contrast to the evolution of Rc, $R, \nabla \phi \otimes \nabla \phi$ and $|\nabla \phi|^{2}$ the evolution equations in Theorem 4.5 for the combinations Rc $-\alpha \nabla \phi \otimes \nabla \phi$ and $R-\alpha|\nabla \phi|^{2}$ do not depend on the intrinsic curvature of $N$.

COROLlary 4.7. - For a solution $(g(t), \phi(t))$ of $(R H)_{\alpha}$ with a time-dependent coupling function $\alpha(t)$, we get

$$
\begin{aligned}
\frac{\partial}{\partial t} S & =\triangle S+2\left|S_{i j}\right|^{2}+2 \alpha\left|\tau_{g} \phi\right|^{2}-\dot{\alpha}|\nabla \phi|^{2} \\
\frac{\partial}{\partial t} S_{i j} & =\triangle_{L} S_{i j}+2 \alpha \tau_{g} \phi \nabla_{i} \nabla_{j} \phi-\dot{\alpha} \nabla_{i} \phi \nabla_{j} \phi .
\end{aligned}
$$

\section{First results about singularities}

In this section, we often use the weak maximum principle which states that for parabolic partial differential equations with a reaction term a solution of the corresponding ODE yields pointwise bounds for the solutions of the PDE. Since we work on an evolving manifold, we need a slightly generalized version. The following result is proved in [6, Theorem 4.4].

Proposition 5.1. - Let $u: M \times[0, T) \rightarrow \mathbb{R}$ be a smooth function satisfying

$$
\frac{\partial}{\partial t} u \geq \triangle_{g(t)} u+\langle X(t), \nabla u\rangle_{g(t)}+F(u),
$$

where $g(t)$ is a smooth 1-parameter family of metrics on $M, X(t)$ a smooth 1-parameter family of vector fields on $M$, and $F: \mathbb{R} \rightarrow \mathbb{R}$ is a locally Lipschitz function. Suppose that $u(\cdot, 0)$ is bounded below by a constant $C_{0} \in \mathbb{R}$ and let $\phi(t)$ be a solution to

$$
\frac{\partial}{\partial t} \phi=F(\phi), \quad \phi(0)=C_{0} .
$$

Then $u(x, t) \geq \phi(t)$ for all $x \in M$ and all $t \in[0, T)$ for which $\phi(t)$ exists. 
Similarly, if (5.1) is replaced by $\frac{\partial}{\partial t} u \leq \triangle_{g(t)} u+\langle X(t), \nabla u\rangle_{g(t)}+F(u)$ and $u(\cdot, 0)$ is bounded from above by $C_{0}$, then $u(x, t) \leq \phi(t)$ for all $x \in M$ and $t \in[0, T)$ for which the solution $\phi(t)$ of the corresponding ODE exists.

Using this, an immediate consequence of Corollary 4.7 is the following.

Corollary 5.2. - Let $(g(t), \phi(t))$ be a solution to the $(R H)_{\alpha}$ flow with a nonnegative, non-increasing coupling function $\alpha(t)$. Let $S(t)=R(g(t))-\alpha(t)|\nabla \phi(t)|_{g(t)}^{2}$ as above, with initial data $S(0)>0$ on $M$. Then $R_{\min }(t):=\min _{x \in M} R(x, t) \rightarrow \infty$ in finite time and thus $g(t)$ must become singular in finite time $T_{\text {sing }} \leq \frac{m}{2 S_{\min }(0)}<\infty$.

Proof. - Since $\alpha(t) \geq 0$ and $\dot{\alpha}(t) \leq 0$ for all $t \geq 0$, Corollary 4.7 yields

$$
\frac{\partial}{\partial t} S \geq \triangle S+2\left|S_{i j}\right|^{2} \geq \triangle S+\frac{2}{m} S^{2}
$$

and thus by comparing with solutions of the ODE $\frac{d}{d t} a(t)=\frac{2}{m} a(t)^{2}$ which are

$$
a(t)=\frac{a(0)}{1-\frac{2 t}{m} a(0)}
$$

the maximum principle above, yields

$$
S_{\min }(t) \geq \frac{S_{\min }(0)}{1-\frac{2 t}{m} S_{\min }(0)}
$$

for all $t \geq 0$ as long as the flow exists. In particular, if $S_{\min }(0)>0$ this implies that $S_{\min }(t) \rightarrow \infty$ in finite time $T_{0} \leq \frac{m}{2 S_{\min }(0)}<\infty$. Since $R=S+\alpha|\nabla \phi|^{2} \geq S$, we find that also $R_{\min }(t) \rightarrow \infty$ before $T_{0}$ and thus $g(t)$ has to become singular in finite time $T_{\text {sing }} \leq T_{0} \leq \frac{m}{2 S_{\min }(0)}<\infty$.

As a second consequence, we see that if the energy density $e(\phi)=\frac{1}{2}|\nabla \phi|^{2}$ blows up at some point in space-time while $\alpha(t)$ is bounded away from zero, then also $g(t)$ must become singular at this point.

COROllary 5.3. - Let $(g(t), \phi(t))_{t \in[0, T)}$ be a smooth solution of $(R H)_{\alpha}$ with a nonincreasing coupling function $\alpha(t)$ satisfying $\alpha(t) \geq \underline{\alpha}>0$ for all $t \in[0, T)$. Suppose that $|\nabla \phi|^{2}\left(x_{k}, t_{k}\right) \rightarrow \infty$ for a sequence $\left(x_{k}, t_{k}\right)_{k \in \mathbb{N}}$ with $t_{k} \nearrow T$. Then also $R\left(x_{k}, t_{k}\right) \rightarrow \infty$ for this sequence and thus $g\left(t_{k}\right)$ must become singular as $t_{k}$ approaches $T$.

Proof. - From (5.2) we obtain $S \geq S_{\min }(0)$ and thus

$$
R=\alpha|\nabla \phi|^{2}+S \geq \underline{\alpha}|\nabla \phi|^{2}+S_{\min }(0), \quad \forall(x, t) \in M \times[0, T) .
$$

Hence, if $|\nabla \phi|^{2}\left(x_{k}, t_{k}\right) \rightarrow \infty$ for a sequence $\left(x_{k}, t_{k}\right)_{k \in \mathbb{N}} \subset M \times[0, T)$ with $t_{k} \nearrow T$ then also $R\left(x_{k}, t_{k}\right) \rightarrow \infty$ for this sequence and $g\left(t_{k}\right)$ must become singular as $t_{k} \nearrow T$.

REMARK 5.4. - The proof shows that Corollary 5.3 stays true if $\alpha(t) \searrow 0$ as $t \nearrow T$ as long as $|\nabla \phi|^{2}\left(x_{k}, t_{k}\right) \rightarrow \infty$ fast enough such that $\alpha\left(t_{k}\right)|\nabla \phi|^{2}\left(x_{k}, t_{k}\right) \rightarrow \infty$ still holds true. 
Now, we derive for $t>0$ an improved version of (5.4) which does not depend on the initial data $S(0)$. Using (5.2) and the maximum principle, we see that if $S_{\min }(0) \geq C \in \mathbb{R}$ we obtain

$$
S_{\min }(t) \geq \frac{C}{1-\frac{2 t}{m} C} \longrightarrow-\frac{m}{2 t} \quad(C \rightarrow-\infty)
$$

and thus $S(t) \geq-\frac{m}{2 t}$ for all $t>0$ as long as the flow exists, independent of $S(0)$. More rigorously, this is obtained as follows. The inequality (5.2) implies

$$
\frac{\partial}{\partial t}(t S)=S+t\left(\frac{\partial}{\partial t} S\right) \geq \triangle(t S)+S\left(1+\frac{2 t}{m} S\right) .
$$

If $\left(x_{0}, t_{0}\right)$ is a point where $t S$ first reaches its minimum over $M \times[0, T-\delta], \delta>0$ arbitrarily small, we get $S\left(x_{0}, t_{0}\right)\left(1+\frac{2 t_{0}}{m} S\left(x_{0}, t_{0}\right)\right) \leq 0$, which is only possible for $t_{0} S\left(x_{0}, t_{0}\right) \geq-\frac{m}{2}$. Hence $t S \geq-\frac{m}{2}$ on all of $M \times[0, T-\delta]$. Since $\delta$ was arbitrary, we obtain the desired inequality $S(t) \geq-\frac{m}{2 t}$ everywhere on $M \times(0, T)$. This yields

$$
R \geq \alpha|\nabla \phi|^{2}-\frac{m}{2 t} \geq \underline{\alpha}|\nabla \phi|^{2}-\frac{m}{2 t}, \quad \forall(x, t) \in M \times(0, T),
$$

which immediately implies the following converse of Corollary 5.3.

Corollary 5.5. - Let $(g(t), \phi(t))_{t \in[0, T)}$ be a smooth solution of $(R H)_{\alpha}$ with a nonincreasing coupling function $\alpha(t) \geq \underline{\alpha}>0$ for all $t \in[0, T)$. Assume that $R \leq R_{0}$ on $M \times[0, T)$.

Then

$$
|\nabla \phi|^{2} \leq \frac{R_{0}}{\underline{\alpha}}+\frac{m}{2 \underline{\alpha} t}, \quad \forall(x, t) \in M \times(0, T) .
$$

Singularities of the type as in Corollary 5.3, where the energy density of $\phi$ blows up, can not only be ruled out if the curvature of $M$ stays bounded. There is also a way to rule them out a-priori. Namely, such singularities cannot form if either $N$ has non-positive sectional curvatures or if we choose the coupling constants $\alpha(t)$ large enough such that

$$
\max _{y \in N}{ }^{N} K(y) \leq \frac{\alpha}{m} \text {. }
$$

Here ${ }^{N} K$ denotes the sectional curvature of $N$. More precisely, we have the following estimates for the energy density $|\nabla \phi|^{2}$.

Proposition 5.6. - Let $(g(t), \phi(t))_{t \in[0, T)}$ be a solution of $(R H)_{\alpha}$ with a non-increasing $\alpha(t) \geq 0$ and let the sectional curvature of $N$ be bounded above by ${ }^{N} K \leq c_{0}$. Then

i) if $N$ has non-positive sectional curvatures or more generally if $c_{0}-\frac{\alpha(t)}{m} \leq 0$, the energy density of $\phi$ is bounded by its initial data,

$$
|\nabla \phi(x, t)|^{2} \leq \max _{y \in M}|\nabla \phi(y, 0)|^{2}, \quad \forall(x, t) \in M \times[0, T) .
$$

ii) if $N$ has non-positive sectional curvatures and $\alpha(t) \geq \underline{\alpha}>0$, we have in addition to (5.6) the estimate

$$
|\nabla \phi(x, t)|^{2} \leq \frac{m}{2 \underline{\alpha} t}, \quad \forall(x, t) \in M \times(0, T) .
$$

iii) in general, the energy density satisfies

$$
|\nabla \phi(x, t)|^{2} \leq 2 \max _{y \in M}|\nabla \phi(y, 0)|^{2}, \quad \forall(x, t) \in M \times\left[0, T^{*}\right),
$$

where $T^{*}:=\min \left\{T,\left(4 c_{0} \max _{y \in M}|\nabla \phi(y, 0)|^{2}\right)^{-1}\right\}$. 
Proof. - This is a consequence of the evolution equation (4.12) and the Cauchy-Schwarz inequality

$$
\frac{1}{m}|\nabla \phi|^{4}=\frac{1}{m}\left|g^{i j} \nabla_{i} \phi \nabla_{j} \phi\right|^{2} \leq\left|\nabla_{i} \phi \nabla_{j} \phi\right|^{2} \leq|\nabla \phi|^{4} .
$$

i) If $N$ has non-positive sectional curvatures, $\left\langle{ }^{N} \operatorname{Rm}\left(\nabla_{i} \phi, \nabla_{j} \phi\right) \nabla_{j} \phi, \nabla_{i} \phi\right\rangle \leq 0$, the evolution equation (4.12) implies

$$
\frac{\partial}{\partial t}|\nabla \phi|^{2} \leq \triangle|\nabla \phi|^{2}
$$

If $c_{0}-\frac{\alpha(t)}{m} \leq 0$, we have

$$
2\left\langle{ }^{N} \operatorname{Rm}\left(\nabla_{i} \phi, \nabla_{j} \phi\right) \nabla_{j} \phi, \nabla_{i} \phi\right\rangle \leq 2 c_{0}|\nabla \phi|^{4} \leq 2 \frac{\alpha}{m}|\nabla \phi|^{4} \leq 2 \alpha\left|\nabla_{i} \phi \nabla_{j} \phi\right|^{2},
$$

and we get again (5.10) from (4.12). The claim now follows from the maximum principle applied to (5.10).

ii) If $\left\langle{ }^{N} \operatorname{Rm}\left(\nabla_{i} \phi, \nabla_{j} \phi\right) \nabla_{j} \phi, \nabla_{i} \phi\right\rangle \leq 0$, (4.12) and (5.9) imply

$$
\frac{\partial}{\partial t}|\nabla \phi|^{2} \leq \triangle|\nabla \phi|^{2}-2 \alpha\left|\nabla_{i} \phi \nabla_{j} \phi\right|^{2} \leq \triangle|\nabla \phi|^{2}-2 \frac{\alpha}{m}|\nabla \phi|^{4} .
$$

We obtain

$$
\frac{\partial}{\partial t}\left(t|\nabla \phi|^{2}\right)=|\nabla \phi|^{2}+t\left(\frac{\partial}{\partial t}|\nabla \phi|^{2}\right) \leq \triangle\left(t|\nabla \phi|^{2}\right)+|\nabla \phi|^{2}\left(1-2 t \frac{\alpha}{m}|\nabla \phi|^{2}\right) .
$$

At the first point $\left(x_{0}, t_{0}\right)$ where $t|\nabla \phi|^{2}$ reaches its maximum over $M \times[0, T-\delta], \delta>0$ arbitrary, we find $1-2 t_{0} \frac{\alpha}{m}|\nabla \phi|^{2}\left(x_{0}, t_{0}\right) \geq 0$, i.e.

$$
t_{0}|\nabla \phi|^{2}\left(x_{0}, t_{0}\right) \leq \frac{m}{2 \alpha} \leq \frac{m}{2 \underline{\alpha}},
$$

which implies that $t|\nabla \phi|^{2} \leq \frac{m}{2 \alpha}$ for every $(x, t) \in M \times[0, T-\delta]$. The claim follows.

iii) From (4.12), we get

$$
\frac{\partial}{\partial t}|\nabla \phi|^{2} \leq \triangle|\nabla \phi|^{2}+2 c_{0}|\nabla \phi|^{4} .
$$

By comparing with solutions of the ODE $\frac{d}{d t} a(t)=2 c_{0} a(t)^{2}$, which are

$$
a(t)=\frac{a(0)}{1-2 c_{0} a(0) t}, \quad t \leq \frac{1}{2 c_{0} a(0)},
$$

the maximum principle from Proposition 5.1 implies

$$
|\nabla \phi(x, t)|^{2} \leq \frac{\max _{y \in M}|\nabla \phi(y, 0)|^{2}}{1-2 c_{0} \max _{y \in M}|\nabla \phi(y, 0)|^{2} t},
$$

for all $x \in M$ and $t \leq \min \left\{T,\left(2 c_{0} \max _{y \in M}|\nabla \phi(y, 0)|^{2}\right)^{-1}\right\}$. In particular, this proves the doubling-time estimate that we claimed.

\section{Gradient estimates and long-time existence}

For solutions $(g(t), \phi(t))$ of the $(R H)_{\alpha}$ flow with non-increasing $\alpha(t) \geq \underline{\alpha}>0$, we have seen in Corollary 5.5 that a uniform bound on the curvature of $(M, g(t))$ implies a uniform bound on $|\nabla \phi|^{2}$. Therefore, one expects that a uniform curvature bound suffices to show long-time existence for our flow. The proof of this result is the main goal of this section. 


\subsection{Evolution equations for $\mathrm{Rm}$ and $\nabla^{2} \phi$}

With $\frac{\partial}{\partial t} g_{i j}=h_{i j}:=-2 R_{i j}+2 \alpha \nabla_{i} \phi^{\mu} \nabla_{j} \phi^{\mu}$, we find the evolution equation for the Christoffel symbols

$$
\begin{aligned}
\frac{\partial}{\partial t} \Gamma_{i j}^{p} & =\frac{1}{2} g^{p q}\left(\nabla_{i} h_{j q}+\nabla_{j} h_{i q}-\nabla_{q} h_{i j}\right) \\
& =g^{p q}\left(-\nabla_{i} R_{j q}-\nabla_{j} R_{i q}+\nabla_{q} R_{i j}\right)+2 \alpha \nabla_{i} \nabla_{j} \phi \nabla^{p} \phi .
\end{aligned}
$$

With this, an elementary computation yields the following evolution equation for the Riemannian curvature tensor (see [28, Proposition 3.2] for a detailed proof).

Proposition 6.1. - Let $(g(t), \phi(t))_{t \in[0, T)}$ be a solution of $(R H)_{\alpha}$. Then the Riemann tensor satisfies

$$
\begin{aligned}
\frac{\partial}{\partial t} R_{i j k \ell}= & \nabla_{i} \nabla_{k} R_{j \ell}-\nabla_{i} \nabla_{\ell} R_{j k}-\nabla_{j} \nabla_{k} R_{i \ell}+\nabla_{j} \nabla_{\ell} R_{i k}-R_{i j q \ell} R_{k q}-R_{i j k q} R_{\ell q} \\
& +2 \alpha\left(\nabla_{i} \nabla_{k} \phi \nabla_{j} \nabla_{\ell} \phi-\nabla_{i} \nabla_{\ell} \phi \nabla_{j} \nabla_{k} \phi-\left\langle{ }^{N} \operatorname{Rm}\left(\nabla_{i} \phi, \nabla_{j} \phi\right) \nabla_{k} \phi, \nabla_{\ell} \phi\right\rangle\right) .
\end{aligned}
$$

REMARK 6.2. - Taking the trace of (6.2), we obtain (4.10), using the twice traced second Bianchi identity. This gives an alternative proof of Proposition 4.3.

If we set $\alpha=0$ in (6.2), we obtain the evolution equation for the curvature tensor under the Ricci flow. It is well-known that this evolution equation can be written in a nicer form, in which its parabolic nature is more apparent. In [15, Lemma 7.2], Hamilton proved

$$
\begin{aligned}
& \quad \nabla_{i} \nabla_{k} R_{j \ell}-\nabla_{i} \nabla_{\ell} R_{j k}-\nabla_{j} \nabla_{k} R_{i \ell}+\nabla_{j} \nabla_{\ell} R_{i k} \\
& \quad=\triangle R_{i j k \ell}+2\left(B_{i j k \ell}-B_{i j \ell k}-B_{i \ell j k}+B_{i k j \ell}\right)-R_{p j k \ell} R_{p i}-R_{i p k \ell} R_{p j},
\end{aligned}
$$

where $B_{i j k \ell}:=R_{i p j q} R_{k p \ell q}$. Plugging this into (6.2) yields the following corollary.

COROLlary 6.3. - Along the $(R H)_{\alpha}$ flow, the Riemannian curvature tensor evolves by

$$
\begin{aligned}
\frac{\partial}{\partial t} R_{i j k \ell}= & \triangle R_{i j k \ell}+2\left(B_{i j k \ell}-B_{i j \ell k}-B_{i \ell j k}+B_{i k j \ell}\right) \\
& -\left(R_{p j k \ell} R_{p i}+R_{i p k \ell} R_{p j}+R_{i j p \ell} R_{p k}+R_{i j k p} R_{p \ell}\right) \\
& +2 \alpha\left(\nabla_{i} \nabla_{k} \phi \nabla_{j} \nabla_{\ell} \phi-\nabla_{i} \nabla_{\ell} \phi \nabla_{j} \nabla_{k} \phi-\left\langle{ }^{N} \operatorname{Rm}\left(\nabla_{i} \phi, \nabla_{j} \phi\right) \nabla_{k} \phi, \nabla_{\ell} \phi\right\rangle\right) .
\end{aligned}
$$

There is a useful convention for writing such equations in a short form.

Definition 6.4. - For two quantities $A$ and $B$, we denote by $A * B$ any quantity obtained from $A \otimes B$ by summation over pairs of matching (Latin and Greek) indices, contractions with the metrics $g$ and $\gamma$ and their inverses, and multiplication with constants depending only on $m=\operatorname{dim} M, n=\operatorname{dim} N$ and the ranks of $A$ and $B$. We also write $(A)^{* 1}:=1 * A,(A)^{* 2}=A * A$, etc.

This notation allows us to write (6.1) and (6.4) in the short forms

$$
\frac{\partial}{\partial t} \Gamma=(\nabla \mathrm{Rm})^{* 1}+\alpha \nabla^{2} \phi * \nabla \phi
$$

and

$$
\frac{\partial}{\partial t} \mathrm{Rm}=\triangle \mathrm{Rm}+(\mathrm{Rm})^{* 2}+\alpha\left(\nabla^{2} \phi\right)^{* 2}+\alpha^{N} \mathrm{Rm} *(\nabla \phi)^{* 4}
$$


It is now easy to compute the evolution of the length of the Riemann tensor. Together with $\frac{\partial}{\partial t} g^{-1}=(\mathrm{Rm})^{* 1}+\alpha(\nabla \phi)^{* 2}$, the above formula yields

$$
\begin{aligned}
\frac{\partial}{\partial t}|\mathrm{Rm}|^{2}= & \left(\frac{\partial}{\partial t} g^{-1}\right) * \mathrm{Rm} * \mathrm{Rm}+2 R_{i j k \ell}\left(\frac{\partial}{\partial t} R_{i j k \ell}\right) \\
= & \triangle|\mathrm{Rm}|^{2}-2|\nabla \mathrm{Rm}|^{2}+(\mathrm{Rm})^{* 3}+\alpha(\mathrm{Rm})^{* 2} *(\nabla \phi)^{* 2} \\
& +\alpha \mathrm{Rm} *\left(\nabla^{2} \phi\right)^{* 2}+\alpha \mathrm{Rm} *{ }^{N} \mathrm{Rm} *(\nabla \phi)^{* 4} .
\end{aligned}
$$

COROLlary 6.5. - Along the $(\mathrm{RH})_{\alpha}$ flow, the Riemannian curvature tensor satisfies

$$
\begin{aligned}
\frac{\partial}{\partial t}|\mathrm{Rm}|^{2} \leq & \triangle|\mathrm{Rm}|^{2}-2|\nabla \mathrm{Rm}|^{2}+C|\mathrm{Rm}|^{3}+\alpha C|\nabla \phi|^{2}|\mathrm{Rm}|^{2} \\
& +\alpha C\left|\nabla^{2} \phi\right|^{2}|\mathrm{Rm}|+\alpha C c_{0}|\nabla \phi|^{4}|\mathrm{Rm}|
\end{aligned}
$$

for constants $C \geq 0$ depending only on the dimension of $M$ and $c_{0}=c_{0}(N) \geq 0$ depending only on the curvature of $N$. If $N$ is flat, we can choose $c_{0}=0$.

Proof. - Follows directly from (6.7) and the fact that $\left|{ }^{N} \mathrm{Rm}\right|$ is bounded on compact $N$.

For the evolution equation for the Hessian of $\phi$, it is important that we do not use the $*$-notation directly. Indeed, we will see that all the terms containing derivatives of the curvature of $M$ cancel each other (using the second Bianchi identity), a phenomenon which cannot be seen when working with the $*$-notation.

A short computation using (A.1) and (A.2) shows that the commutator $\left[\nabla_{i} \nabla_{j}, \triangle\right] \phi^{\lambda}=$ $\nabla_{i} \nabla_{j} \tau_{g} \phi^{\lambda}-\triangle \nabla_{i} \nabla_{j} \phi^{\lambda}$ is given by

$$
\begin{aligned}
{\left[\nabla_{i} \nabla_{j}, \triangle\right] \phi^{\lambda}=} & \nabla_{k} R_{j p i k} \nabla_{p} \phi^{\lambda}+2 R_{i k j p} \nabla_{k} \nabla_{p} \phi^{\lambda} \\
& -R_{i p} \nabla_{j} \nabla_{p} \phi^{\lambda}-\nabla_{i} R_{j p} \nabla_{p} \phi^{\lambda}-R_{j p} \nabla_{i} \nabla_{p} \phi^{\lambda} \\
& +\left({ }^{N} \operatorname{Rm} * \nabla^{2} \phi *(\nabla \phi)^{* 2}+\left(\partial^{N} \mathrm{Rm}\right) *(\nabla \phi)^{* 4}\right)_{i j},
\end{aligned}
$$

see [28, equation (3.10)] for details. With (6.1) we continue

$$
\begin{aligned}
{\left[\nabla_{i} \nabla_{j}, \triangle\right] \phi^{\lambda}-\left(\frac{\partial}{\partial t} \Gamma_{i j}^{k}\right) \nabla_{k} \phi^{\lambda}=} & \left(\mathrm{Rm} * \nabla^{2} \phi^{\lambda}\right)_{i j}-2 \alpha \nabla_{i} \nabla_{j} \phi \nabla_{k} \phi \nabla_{k} \phi^{\lambda} \\
& +\left({ }^{N} \mathrm{Rm} * \nabla^{2} \phi *(\nabla \phi)^{* 2}+\left(\partial^{N} \mathrm{Rm}\right) *(\nabla \phi)^{* 4}\right)_{i j},
\end{aligned}
$$

where we used the second Bianchi identity $\left(\nabla_{k} R_{j p i k}+\nabla_{j} R_{i p}-\nabla_{p} R_{i j}\right) \nabla_{p} \phi^{\lambda}=0$ to cancel all terms containing derivatives of the curvature of $(M, g)$. Since the $\nabla^{2} \phi$ live in different bundles for different times, we work again with the covariant time derivative $\nabla_{t}$ (and with the interpretation of $\nabla^{2} \phi$ as a 2-linear $T N$-valued map along $\tilde{\phi}$ ), as we already did in Section 4, see appendix for details. At the base point of coordinates satisfying (A.5), we find with (A.6) and the remark following it

$$
\begin{aligned}
\nabla_{t}\left(\nabla_{i} \nabla_{j} \phi^{\lambda}\right)= & \nabla_{i} \nabla_{j} \frac{\partial}{\partial t} \phi^{\lambda}-\left(\frac{\partial}{\partial t} \Gamma_{i j}^{k}\right) \nabla_{k} \phi^{\lambda}+{ }^{N} \operatorname{Rm}\left(\frac{\partial}{\partial t} \phi, \nabla_{i} \phi\right) \nabla_{j} \phi^{\lambda} \\
= & \triangle \nabla_{i} \nabla_{j} \phi^{\lambda}+\left(\mathrm{Rm} * \nabla^{2} \phi^{\lambda}\right)_{i j}+\alpha \nabla_{i} \nabla_{j} \phi * \nabla \phi * \nabla \phi^{\lambda} \\
& +\left({ }^{N} \mathrm{Rm} * \nabla^{2} \phi *(\nabla \phi)^{* 2}+\left(\partial^{N} \mathrm{Rm}\right) *(\nabla \phi)^{* 4}\right)_{i j} .
\end{aligned}
$$

$4^{\text {e }}$ SÉRIE - TOME $45-2012-$ No $^{\circ}$ 
With $\triangle\left|\nabla^{2} \phi\right|^{2}=2 \triangle\left(\nabla_{i} \nabla_{j} \phi^{\lambda}\right) \nabla_{i} \nabla_{j} \phi^{\lambda}+2\left|\nabla^{3} \phi\right|^{2}$, we finally obtain

$$
\begin{aligned}
\frac{\partial}{\partial t}\left|\nabla^{2} \phi\right|^{2}= & \left(\frac{\partial}{\partial t} g^{-1}\right) *\left(\nabla^{2} \phi\right)^{* 2}+2 \nabla_{t}\left(\nabla_{i} \nabla_{j} \phi^{\lambda}\right) \nabla_{i} \nabla_{j} \phi^{\lambda} \\
= & \operatorname{Rm} *\left(\nabla^{2} \phi\right)^{* 2}+\alpha(\nabla \phi)^{* 2} *\left(\nabla^{2} \phi\right)^{* 2}+\triangle\left|\nabla^{2} \phi\right|^{2}-2\left|\nabla^{3} \phi\right|^{2} \\
& +{ }^{N} \mathrm{Rm} *\left(\nabla^{2} \phi\right)^{* 2} *(\nabla \phi)^{* 2}+\left(\partial^{N} \mathrm{Rm}\right) *\left(\nabla^{2} \phi\right) *(\nabla \phi)^{* 4} .
\end{aligned}
$$

Since $\left|{ }^{N} \mathrm{Rm}\right|$ and $\left|\partial^{N} \mathrm{Rm}\right|$ are bounded on compact manifolds $N$, say by a constant $c_{1}$, this proves the following proposition.

Proposition 6.6. - Let $(g(t), \phi(t))_{t \in[0, T)}$ be a solution of $(R H)_{\alpha}$. Then the norm of the Hessian of $\phi$ satisfies the estimate

$$
\begin{aligned}
\frac{\partial}{\partial t}\left|\nabla^{2} \phi\right|^{2} \leq & \triangle\left|\nabla^{2} \phi\right|^{2}-2\left|\nabla^{3} \phi\right|^{2}+C|\operatorname{Rm}|\left|\nabla^{2} \phi\right|^{2} \\
& +\alpha C|\nabla \phi|^{2}\left|\nabla^{2} \phi\right|^{2}+C c_{1}|\nabla \phi|^{4}\left|\nabla^{2} \phi\right|+C c_{1}|\nabla \phi|^{2}\left|\nabla^{2} \phi\right|^{2}
\end{aligned}
$$

along the flow for some constants $C=C(m) \geq 0$ and $c_{1}=c_{1}(N) \geq 0$ depending on the dimension $m$ of $M$ and the curvature of $N$, respectively. If $N$ is flat, we may choose $c_{1}=0$.

Remark 6.7. - If we set $\alpha \equiv 0$, Corollary 6.5 and Proposition 6.6 yield the formulas for the Ricci-DeTurck flow $(R H)_{0}$, in particular (6.8) reduces to the well-known evolution inequality

$$
\frac{\partial}{\partial t}|\mathrm{Rm}|^{2} \leq \triangle|\mathrm{Rm}|^{2}-2|\nabla \mathrm{Rm}|^{2}+C|\mathrm{Rm}|^{3}
$$

for the Ricci flow, first derived by Hamilton [15, Corollary 13.3]. Moreover, if $\alpha \equiv 2$ and $N \subseteq \mathbb{R}$ (and thus $c_{0}=c_{1}=0$ ), the estimates (6.8) and (6.12) reduce to the estimates found by List (cf. [22, Lemma 2.15 and 2.16]).

\subsection{Interior-in-time higher order gradient estimates}

Using the evolution equations for the curvature tensor and the Hessian of $\phi$, we get evolution equations for higher order derivatives by induction.

Definition 6.8. - To keep the notation short, we define for $k \geq 0$

$$
\begin{aligned}
I_{k}:= & \sum_{i+j=k} \nabla^{i} \mathrm{Rm} * \nabla^{j} \mathrm{Rm}+\alpha \sum_{A_{k}}\left(\partial^{i N} \mathrm{Rm}+1\right) * \nabla^{j_{1}} \phi * \cdots * \nabla^{j_{\ell}} \phi \\
& +\alpha \sum_{B_{k}} \nabla^{j_{1}} \phi * \cdots * \nabla^{j_{\ell-1}} \phi * \nabla^{j_{\ell}} \mathrm{Rm},
\end{aligned}
$$

where the last two sums are taken over all elements of the index sets defined by

$$
\begin{aligned}
& A_{k}:=\left\{\left(i, j_{1}, \ldots, j_{\ell}\right) \mid 0 \leq i \leq k+1,1 \leq j_{s} \leq k+2 \forall s \text { and } j_{1}+\cdots+j_{\ell}=k+4\right\}, \\
& B_{k}:=\left\{\left(j_{1}, \ldots, j_{\ell}\right) \mid 1 \leq j_{s}<k+2 \forall s<\ell, 0 \leq j_{\ell} \leq k \text { and } j_{1}+\cdots+j_{\ell}=k+2\right\} .
\end{aligned}
$$

Lemma 6.9. - Let $(g(t), \phi(t))_{t \in[0, T)}$ be a solution to the $(R H)_{\alpha}$ flow. Then for $k \geq 0$, with $I_{k}$ defined as in (6.13), we obtain

$$
\frac{\partial}{\partial t} \nabla^{k} \mathrm{Rm}=\triangle \nabla^{k} \mathrm{Rm}+I_{k}
$$

Remark 6.10. - If $(N, \gamma)=(\mathbb{R}, \delta)$, all terms in $I_{k}$ containing $\partial^{i}{ }^{N} \mathrm{Rm}$ vanish, and the result reduces to (a slightly weaker version of) List's result [22, Lemma 2.19]. Note that we do not need all the elements of $A_{k}$ here, but defining $A_{k}$ this way allows us to use the same index set again in Definition 6.11. 
Proof. - From (6.6), we see that (6.14) holds for $k=0$. For the induction step, assume that (6.14) holds for some $k \geq 0$ and compute

$$
\frac{\partial}{\partial t} \nabla^{k+1} \mathrm{Rm}=\frac{\partial}{\partial t}\left(\partial \nabla^{k} \mathrm{Rm}+\Gamma * \nabla^{k} \mathrm{Rm}\right)=\nabla\left(\Delta \nabla^{k} \mathrm{Rm}\right)+\nabla I_{k}+\frac{\partial}{\partial t} \Gamma * \nabla^{k} \mathrm{Rm} .
$$

Since $\nabla I_{k}$ is of the form $I_{k+1}$ and also $\frac{\partial}{\partial t} \Gamma * \nabla^{k} \mathrm{Rm}=\left(\nabla \mathrm{Rm}+\alpha \nabla^{2} \phi * \nabla \phi\right) * \nabla^{k} \mathrm{Rm}$ appears in $I_{k+1}$, it remains to compute the very first term. With the commutator rule (A.1), we get

$$
\begin{aligned}
\nabla\left(\triangle \nabla^{k} \mathrm{Rm}\right) & =\triangle \nabla^{k+1} \mathrm{Rm}+\nabla \mathrm{Rm} * \nabla^{k} \mathrm{Rm}+\mathrm{Rm} * \nabla^{k+1} \mathrm{Rm} \\
& =\triangle \nabla^{k+1} \mathrm{Rm}+I_{k+1} .
\end{aligned}
$$

Similar to (6.7), we obtain

$$
\begin{aligned}
\frac{\partial}{\partial t}\left|\nabla^{k} \mathrm{Rm}\right|^{2}= & \left(\frac{\partial}{\partial t} g^{-1}\right) * \nabla^{k} \mathrm{Rm} * \nabla^{k} \mathrm{Rm}+2 \nabla^{k} \mathrm{Rm}\left(\frac{\partial}{\partial t} \nabla^{k} \mathrm{Rm}\right) \\
= & \mathrm{Rm} *\left(\nabla^{k} \mathrm{Rm}\right)^{* 2}+\alpha(\nabla \phi)^{* 2} *\left(\nabla^{k} \mathrm{Rm}\right)^{* 2} \\
& +2 \nabla^{k} \mathrm{Rm}\left(\triangle \nabla^{k} \mathrm{Rm}\right)+\nabla^{k} \mathrm{Rm} * I_{k} .
\end{aligned}
$$

Hence, using the fact that $\mathrm{Rm} * \nabla^{k} \mathrm{Rm}$ as well as $\alpha(\nabla \phi)^{* 2} * \nabla^{k} \mathrm{Rm}$ are already contained in $I_{k}$, we find

$$
\frac{\partial}{\partial t}\left|\nabla^{k} \mathrm{Rm}\right|^{2}=\triangle\left|\nabla^{k} \mathrm{Rm}\right|^{2}-2\left|\nabla^{k+1} \mathrm{Rm}\right|^{2}+\nabla^{k} \mathrm{Rm} * I_{k} .
$$

Definition 6.11. - To compute the higher order derivatives of $\phi$, we define

$$
\begin{aligned}
J_{k}:= & \sum_{i+j=k} \nabla^{i} \mathrm{Rm} * \nabla^{j+2} \phi+\sum_{A_{k}}\left(\partial^{i N^{N} \mathrm{Rm}}+1\right) * \nabla^{j_{1}} \phi * \cdots * \nabla^{j_{\ell}} \phi \\
& +\alpha \sum_{B_{k}} \nabla^{j_{1}} \phi * \cdots * \nabla^{j_{\ell-1}} \phi * \nabla^{j_{\ell}+2} \phi,
\end{aligned}
$$

with $A_{k}$ and $B_{k}$ defined as in Definition 6.8.

Lemma 6.12. - Let $(g(t), \phi(t))_{t \in[0, T)}$ be a solution to the $(R H)_{\alpha}$ flow. Then for $k \geq 0$, with $J_{k}$ defined as in (6.16), we have

$$
\nabla_{t}\left(\nabla^{k+2} \phi\right)=\triangle \nabla^{k+2} \phi+J_{k} .
$$

Proof. - For $k=0$, the statement holds by (6.10). For the induction step, we use again the interpretation of $\nabla^{k} \phi$ as a $k$-linear $T N$-valued map along $\tilde{\phi}$ and compute analogously to (A.6) and the remark following it

$$
\begin{aligned}
\nabla_{t}\left(\nabla^{k+3} \phi\right) & =\nabla \nabla_{t}\left(\nabla^{k+2} \phi\right)+\frac{\partial}{\partial t} \Gamma * \nabla^{k+2} \phi+{ }^{N} \operatorname{Rm}\left(\frac{\partial}{\partial t} \phi, \nabla \phi\right) \nabla^{k+2} \phi \\
& =\nabla\left(\triangle \nabla^{k+2} \phi\right)+\nabla J_{k}+\frac{\partial}{\partial t} \Gamma * \nabla^{k+2} \phi+{ }^{N} \mathrm{Rm} * \nabla^{2} \phi * \nabla \phi * \nabla^{k+2} \phi .
\end{aligned}
$$

Again, we only have to look at the first term, since $\nabla J_{k},{ }^{N} \mathrm{Rm} * \nabla^{2} \phi * \nabla \phi * \nabla^{k+2} \phi$ and $\frac{\partial}{\partial t} \Gamma * \nabla^{k+2} \phi=\left(\nabla \mathrm{Rm}+\alpha \nabla^{2} \phi * \nabla \phi\right) * \nabla^{k+2} \phi$ are obviously of the form $J_{k+1}$. With a 
higher order analog to (A.3), we obtain

$$
\begin{aligned}
\nabla\left(\triangle \nabla^{k+2} \phi\right)= & \nabla_{p} \nabla \nabla_{p} \nabla^{k+2} \phi+\mathrm{Rm} * \nabla^{k+3} \phi+{ }^{N} \mathrm{Rm} * \nabla^{k+3} \phi * \nabla \phi * \nabla \phi \\
= & \nabla_{p} \nabla_{p} \nabla^{k+3} \phi+\nabla \mathrm{Rm} * \nabla^{k+2} \phi+\mathrm{Rm} * \nabla^{k+3} \phi \\
& +\left(\partial^{N} \mathrm{Rm}\right) * \nabla^{k+2} \phi *(\nabla \phi)^{* 3}+{ }^{N} \mathrm{Rm} * \nabla^{k+3} \phi *(\nabla \phi)^{* 2} \\
& +{ }^{N} \mathrm{Rm} * \nabla^{k+3} \phi * \nabla^{2} \phi * \nabla \phi \\
= & \triangle \nabla^{k+3} \phi+J_{k+1},
\end{aligned}
$$

and the claim follows.

As in (6.11), we compute

$$
\begin{aligned}
\frac{\partial}{\partial t}\left|\nabla^{k+2} \phi\right|^{2}= & \left(\frac{\partial}{\partial t} g^{-1}\right) * \nabla^{k+2} \phi * \nabla^{k+2} \phi+2 \nabla^{k+2} \phi^{\lambda} \nabla_{t}\left(\nabla^{k+2} \phi^{\lambda}\right) \\
= & \operatorname{Rm} *\left(\nabla^{k+2} \phi\right)^{* 2}+\alpha(\nabla \phi)^{* 2} *\left(\nabla^{k+2} \phi\right)^{* 2} \\
& +2 \nabla^{k+2} \phi^{\lambda}\left(\triangle \nabla^{k+2} \phi^{\lambda}\right)+\nabla^{k+2} \phi * J_{k} \\
= & 2 \nabla^{k+2} \phi^{\lambda}\left(\triangle \nabla^{k+2} \phi^{\lambda}\right)+\nabla^{k+2} \phi * J_{k} .
\end{aligned}
$$

With $\triangle\left|\nabla^{k+2} \phi\right|^{2}=2 \nabla^{k+2} \phi^{\lambda}\left(\triangle \nabla^{k+2} \phi^{\lambda}\right)+2\left|\nabla^{k+3} \phi\right|^{2}$, we finally find

$$
\frac{\partial}{\partial t}\left|\nabla^{k+2} \phi\right|^{2}=\triangle\left|\nabla^{k+2} \phi\right|^{2}-2\left|\nabla^{k+3} \phi\right|^{2}+\nabla^{k+2} \phi * J_{k} .
$$

The next trick is to combine the two equations (6.15) and (6.18) to a single equation. Remember that we already used a similar idea in Section 4, where we combined the evolution equations of Rc and $\nabla \phi \otimes \nabla \phi$ (respectively $R$ and $|\nabla \phi|^{2}$ ) to a single equation for a combined quantity $S_{i j}$ (respectively $S$ ), which was much more convenient to deal with. Here, we define the "vector"

$$
\mathcal{T}=\left(\mathrm{Rm}, \nabla^{2} \phi\right) \in \Gamma\left(\left(T^{*} M\right)^{\otimes 4}\right) \times \Gamma\left(\left(T^{*} M\right)^{\otimes 2} \otimes \phi^{*} T N\right)
$$

with norm $|\mathcal{T}|^{2}=|\mathrm{Rm}|^{2}+\left|\nabla^{2} \phi\right|^{2}$ and derivatives $\nabla^{k} \mathcal{T}=\left(\nabla^{k} \mathrm{Rm}, \nabla^{k+2} \phi\right)$. Combining the evolution equations (6.15) and (6.18), we get

$$
\frac{\partial}{\partial t}\left|\nabla^{k} \mathcal{T}\right|^{2}=\triangle\left|\nabla^{k} \mathcal{T}\right|^{2}-2\left|\nabla^{k+1} \mathcal{J}\right|^{2}+L_{k},
$$

where $L_{k}:=\nabla^{k} \mathrm{Rm} * I_{k}+\nabla^{k+2} \phi * J_{k}$. We can now apply Bernstein's ideas [2] to obtain interior-in-time estimates for all derivatives $\left|\nabla^{k} \mathcal{T}\right|^{2}$ via an induction argument. For the Ricci flow, this was independently done by Bando [1] and Shi [38].

Theorem 6.13. - Let $(g(t), \phi(t))_{t \in[0, T)}$ solve $(R H)_{\alpha}$ with non-increasing $\alpha(t) \in[\underline{\alpha}, \bar{\alpha}]$, $0<\underline{\alpha} \leq \bar{\alpha}<\infty$ and $T<\infty$. Let the Riemannian curvature tensor of $M$ be uniformly bounded along the flow, $|\mathrm{Rm}| \leq R_{0}$. Then there exists a constant $K=K\left(\underline{\alpha}, \bar{\alpha}, R_{0}, T, m, N\right)<\infty$ such that the following two estimates hold

$$
\begin{aligned}
|\nabla \phi|^{2} & \leq \frac{K}{t}, \quad \forall(x, t) \in M \times(0, T), \\
|\mathcal{T}|^{2} & =|\mathrm{Rm}|^{2}+\left|\nabla^{2} \phi\right|^{2} \leq \frac{K^{2}}{t^{2}}, \quad \forall(x, t) \in M \times(0, T) .
\end{aligned}
$$

Moreover, there exist constants $C_{k}$ depending on $k, \bar{\alpha}, m$ and $N$, such that

$$
\left|\nabla^{k} \mathcal{T}\right|^{2}=\left|\nabla^{k} \mathrm{Rm}\right|^{2}+\left|\nabla^{k+2} \phi\right|^{2} \leq C_{k}\left(\frac{K}{t}\right)^{k+2}, \quad \forall(x, t) \in M \times(0, T) .
$$


Proof. - Since the method of proof is quite standard, we only give a brief sketch of the argument and refer to the authors thesis [28, Theorem 3.10] for more details. Setting $1 \leq K_{1}:=\max \left\{\frac{2 m^{2} R_{0} T+m}{2 \underline{\alpha}}, R_{0} T, 1\right\}<\infty$, we obtain

$$
|\nabla \phi|^{2} \leq \frac{m^{2} R_{0}}{\underline{\alpha}}+\frac{m}{2 \underline{\alpha} t} \leq \frac{K_{1}}{t} \quad \text { and } \quad|\mathrm{Rm}| \leq \frac{K_{1}}{t}, \quad \forall(x, t) \in M \times(0, T)
$$

from Corollary 5.5. In the following, $C$ denotes a constant depending on $K_{1}, \bar{\alpha}, m$ and the geometry of $N$, possibly changing from line to line. With the estimates for $|\operatorname{Rm}|$ and $|\nabla \phi|^{2}$, and using $\left|\nabla^{2} \phi\right| \leq \frac{1}{t}+t\left|\nabla^{2} \phi\right|^{2}$, we obtain for $f(x, t):=t^{2}\left|\nabla^{2} \phi\right|^{2}\left(8 K_{1}+t|\nabla \phi|^{2}\right)$

$$
\begin{aligned}
\left(\frac{\partial}{\partial t}-\triangle\right) f \leq & -2 t^{2}\left|\nabla^{3} \phi\right|^{2}\left(8 K_{1}+t|\nabla \phi|^{2}\right)+\frac{C}{t} f+\frac{C}{t} \cdot 9 K_{1}-2 t^{3}\left|\nabla^{2} \phi\right|^{4}+\frac{C}{t} f \\
& +8 t^{3}\left|\nabla^{3} \phi\right|\left|\nabla^{2} \phi\right| \cdot\left|\nabla^{2} \phi\right||\nabla \phi|
\end{aligned}
$$

on $M \times(0, T)$. The last term can be absorbed by the two negative terms,

$$
\begin{aligned}
8 t^{3}\left|\nabla^{3} \phi\right|\left|\nabla^{2} \phi\right|^{2}|\nabla \phi| & \leq \frac{1}{2}\left(8 K_{1}\right)\left(4 t^{2}\left|\nabla^{3} \phi\right|^{2}\right)+\frac{1}{2}\left(8 K_{1}\right)^{-1}\left(16 t^{4}\left|\nabla^{2} \phi\right|^{4}|\nabla \phi|^{2}\right) \\
& =2 t^{2}\left|\nabla^{3} \phi\right|^{2} \cdot 8 K_{1}+\frac{8 t|\nabla \phi|^{2}}{8 K_{1}} \cdot t^{3}\left|\nabla^{2} \phi\right|^{4} \\
& \leq 2 t^{2}\left|\nabla^{3} \phi\right|^{2}\left(8 K_{1}+t|\nabla \phi|^{2}\right)+t^{3}\left|\nabla^{2} \phi\right|^{4} .
\end{aligned}
$$

Here, we used $\frac{8 t|\nabla \phi|^{2}}{8 K_{1}} \leq 1$ which motivates our choice of the constant $8 K_{1}$ in the definition of $f$. From $\left(\frac{\partial}{\partial t}-\triangle\right) f \leq \frac{C}{t} f+\frac{C}{t}-t^{3}\left|\nabla^{2} \phi\right|^{4} \leq \frac{1}{\left(9 K_{1}\right)^{2} t}\left(C f+C-f^{2}\right)$, we conclude, using $f(\cdot, 0)=0$ and the maximum principle, that $-f^{2}+C f+C \geq 0$. Equivalently, $f \leq D:=\frac{1}{2}\left(C+\sqrt{C^{2}+4 C}\right)$ on $M \times[0, T)$. For positive $t$, this implies

$$
\left|\nabla^{2} \phi\right|^{2}=\frac{f}{t^{2}\left(8 K_{1}+t|\nabla \phi|^{2}\right)} \leq \frac{D}{8 K_{1} t^{2}} \leq\left(\frac{K_{2}}{t}\right)^{2},
$$

where $K_{2}:=\sqrt{D / 8 K_{1}}<\infty$. Setting $K:=K_{1}+K_{2}$, we get (6.21) and (6.22). Using a similar argument, one can then prove (6.23) inductively. The crucial estimates are $L_{k} \leq C\left(\frac{K}{t}\right)^{k+3}$ and

$$
L_{k+1} \leq C\left(\frac{K}{t}\right)^{k+4}+C \frac{K}{t}\left|\nabla^{k+1} \mathcal{T}\right|^{2},
$$

where $C$ now denotes a constant depending only on $\bar{\alpha}, m, N$ and $k$ (but not on $K$ or $T$ ). Defining $h(x, t):=t^{k+3}\left|\nabla^{k+1} \mathcal{J}\right|^{2}\left(\lambda+t^{k+2}\left|\nabla^{k} \mathcal{J}\right|^{2}\right)$ with $\lambda=8 C_{k} K^{k+2}$, these estimates give

$$
\begin{aligned}
\left(\frac{\partial}{\partial t}-\triangle\right) h \leq & -2 t^{k+3}\left|\nabla^{k+2} \mathcal{T}\right|^{2}\left(\lambda+t^{k+2}\left|\nabla^{k} \mathcal{T}\right|^{2}\right)+\frac{C K}{t} h+\frac{C}{t} K^{k+4}\left(\lambda+t^{k+2}\left|\nabla^{k} \mathcal{T}\right|^{2}\right) \\
& -2 t^{2 k+5}\left|\nabla^{k+1} \mathcal{J}\right|^{4}+\frac{C}{t} h+\frac{C}{t} K^{k+3} t^{k+3}\left|\nabla^{k+1} \mathcal{J}\right|^{2} \\
& +8 t^{2 k+5}\left|\nabla^{k+2} \mathcal{J}\right|\left|\nabla^{k+1} \mathcal{T}\right| \cdot\left|\nabla^{k+1} \mathcal{J}\right|\left|\nabla^{k} \mathcal{T}\right| .
\end{aligned}
$$

Using $K \geq 1$, the inductive assumption and Cauchy-Schwarz, we rewrite this as

$$
\begin{aligned}
\left(\frac{\partial}{\partial t}-\triangle\right) h \leq & -2 t^{k+3}\left|\nabla^{k+2} \mathcal{T}\right|^{2}\left(\lambda+t^{k+2}\left|\nabla^{k} \mathcal{T}\right|^{2}\right)-\frac{3}{2} t^{2 k+5}\left|\nabla^{k+1} \mathcal{T}\right|^{4} \\
& +\frac{C K}{t} h+\frac{C}{t} K^{2 k+6}+8 t^{2 k+5}\left|\nabla^{k+2} \mathcal{T}\right|\left|\nabla^{k+1} \mathcal{T}\right|^{2}\left|\nabla^{k} \mathcal{T}\right| .
\end{aligned}
$$

$4^{\mathrm{e}}$ SÉRIE - TOME $45-2012$ - No 1 
Again, the last term can be absorbed by the negative terms

$$
\begin{aligned}
8 t^{2 k+5}\left|\nabla^{k+2} \mathcal{T}\right|\left|\nabla^{k+1} \mathcal{T}\right|^{2}\left|\nabla^{k} \mathcal{T}\right| & \leq \frac{1}{2} \lambda\left(4 t^{k+3}\left|\nabla^{k+2} \mathcal{T}\right|^{2}\right)+\frac{1}{2} \lambda^{-1}\left(16 t^{3 k+7}\left|\nabla^{k+1} \mathcal{T}\right|^{4}\left|\nabla^{k} \mathcal{T}\right|^{2}\right) \\
& =2 t^{k+3}\left|\nabla^{k+2} \mathcal{T}\right|^{2} \cdot \lambda+\frac{8 t^{k+2}\left|\nabla^{k} \mathcal{G}\right|^{2}}{\lambda} \cdot t^{2 k+5}\left|\nabla^{k+1} \mathcal{T}\right|^{4} \\
& \leq 2 t^{k+3}\left|\nabla^{k+2} \mathcal{T}\right|^{2}\left(\lambda+t^{k+2}\left|\nabla^{k} \mathcal{T}\right|^{2}\right)+t^{2 k+5}\left|\nabla^{k+1} \mathcal{T}\right|^{4},
\end{aligned}
$$

which explains our choice of $\lambda$. A maximum principle argument like the one for $f$ above then yields $-h^{2}+C K^{2 k+5} h+C K^{4 k+10} \geq 0$, i.e., $h \leq \frac{1}{2}\left(C+\sqrt{C^{2}+4 C}\right) K^{2 k+5}=: D K^{2 k+5}$ on $M \times[0, T)$. For $t>0$,

$$
\left|\nabla^{k+1} \mathcal{T}\right|^{2}=\frac{h}{t^{k+3}\left(\lambda+t^{k+2}\left|\nabla^{k} \mathcal{T}\right|^{2}\right)} \leq \frac{D K^{2 k+5}}{t^{k+3} 8 C_{k} K^{k+2}}=C_{k+1}\left(\frac{K}{t}\right)^{k+3},
$$

where $C_{k+1}:=D /\left(8 C_{k}\right)$. This proves the induction step and hence the theorem.

In the following corollary, we state a local version of the gradient estimates. The setting is made in such a way to perfectly fit the proof of the non-collapsing result in Section 8 .

Corollary 6.14. - Let $(g(t), \phi(t))_{t \in[0, T)}$ solve $(R H)_{\alpha}$ withnon-increasing $\alpha(t) \in[\underline{\alpha}, \bar{\alpha}]$, $0<\underline{\alpha} \leq \bar{\alpha}<\infty$ and $T^{\prime}<T<\infty$. Let $B:=B_{g\left(T^{\prime}\right)}(x, r)$ be a ball around $x$ with radius $r$, measured with respect to the metric at time $T^{\prime}$. Assume that $|\mathrm{Rm}| \leq R_{0}$ on the set $B \times\left[0, T^{\prime}\right)$. Then there exist constants $K=K\left(\underline{\alpha}, \bar{\alpha}, R_{0}, T, m, N\right)<\infty$ and $C_{k}=C_{k}(k, \bar{\alpha}, m, N)$ for $k \in \mathbb{N}, C_{0}=1$, such that the following estimates hold for $k \geq 0$

$$
\begin{aligned}
|\nabla \phi|^{2} & \leq \frac{K}{t} \quad \text { and } \quad|\mathrm{Rm}| \leq \frac{K}{t}, \quad \forall(x, t) \in B^{1 / 2} \times\left(0, T^{\prime}\right), \\
\left|\nabla^{k} \mathcal{G}\right|^{2} & =\left|\nabla^{k} \mathrm{Rm}\right|^{2}+\left|\nabla^{k+2} \phi\right|^{2} \leq C_{k}\left(\frac{K}{t}\right)^{k+2}, \quad \forall(x, t) \in B^{1 / 2} \times\left(0, T^{\prime}\right),
\end{aligned}
$$

where $B^{1 / 2}:=B_{g\left(T^{\prime}\right)}(x, r / 2)$ is the ball of half the radius and the same center as $B$.

Proof. - The statement (6.25) follows exactly as in Theorem 6.13. The induction step is carried out using a cut-off function to ensure that the maxima are attained in the interior of the set $B$. More details can be found in the author's thesis [28, Proposition A.5].

\subsection{Long-time existence}

This subsection follows Section 6.7 about long-time existence for the Ricci flow from Chow and Knopf's book [6]. We first need a technical lemma.

Lemma 6.15. - Let $(g(t), \phi(t))_{t \in[0, T)}$ solve $(R H)_{\alpha}$ with a non-increasing $\alpha(t) \in[\underline{\alpha}, \bar{\alpha}]$, $0<\underline{\alpha} \leq \bar{\alpha}<\infty$ and $T<\infty$. Let the Riemannian curvature tensor of $M$ be uniformly bounded along the flow, $|\mathrm{Rm}| \leq R_{0}$, and fix a background metric $\tilde{g}$. Then for each $k \geq 0$ there exists $a$ constant $C_{k}$ depending on $k, m, N, T, \underline{\alpha}, \bar{\alpha}, R_{0}$ and the initial data $(g(0), \phi(0))$ such that

$$
\left|\tilde{\nabla}^{k} g(x, t)\right|_{\tilde{g}}^{2}+\left|\tilde{\nabla}^{k} \operatorname{Rm}(x, t)\right|_{\tilde{g}}^{2}+\left|\tilde{\nabla}^{k} \phi(x, t)\right|_{\tilde{g}}^{2} \leq C_{k}
$$

for all $(x, t) \in M \times[0, T)$. Here, $\tilde{\nabla}={ }^{\tilde{g}} \nabla$ denotes the Levi-Civita connection with respect to the background metric $\tilde{g}$. 
Proof. - With Theorem 6.9, the proof becomes a straightforward computation, and we therefore only give a sketch. In [6, Section 6.7], all the details are carried out in the case of the Ricci flow and they can easily be adopted to our flow. Since $M$ is closed, there exists a finite atlas for which we have uniform bounds on the derivatives of the local charts. Working in such a chart $\psi: U \rightarrow \mathbb{R}^{m}$, it suffices to derive the desired estimates for the Euclidean metric $\delta$ in $U$ and the ordinary derivatives, since $\tilde{g}$ and $\tilde{\nabla}$ are fixed. In particular, we can interpret $\Gamma$ as a tensor, namely $\Gamma=\Gamma-{ }^{\delta} \Gamma$. On the compact interval $[0, T / 2]$, all the derivatives $\left|\nabla^{k} \phi\right|_{g}^{2}$ and $\left|\nabla^{k} \mathrm{Rm}\right|_{g}^{2}$ are uniformly bounded. On the interval $[T / 2, T)$, Theorem 6.13 above yields uniform bounds for these derivatives. Hence

$$
\left|\nabla^{k} \phi\right|_{g}^{2}+\left|\nabla^{k} \mathrm{Rm}\right|_{g}^{2} \leq \bar{C}_{k}
$$

for some $\bar{C}_{k}<\infty$. In particular, $\phi=\mathrm{Rc}-\alpha \nabla \phi \otimes \nabla \phi$ is uniformly bounded on $[0, T)$. From [6, Lemma 6.49], we infer that all $g(t)$ are uniformly equivalent on $[0, T)$, and thus for some constant $C$

$$
C^{-1} \delta \leq g(x, t) \leq C \delta, \quad \forall(x, t) \in U \times[0, T) .
$$

With $\frac{\partial}{\partial t}(\partial g)=\partial\left(\frac{\partial}{\partial t} g\right)=-2 \partial \phi=-2(\nabla \phi+\Gamma * \varnothing)$, we compute

$$
\left|\frac{\partial}{\partial t} \partial g\right|_{\delta} \leq C\left|\frac{\partial}{\partial t} \partial g\right| \leq C|\nabla \phi|+C|\Gamma||\phi| \text {. }
$$

Thus, we have

$$
\left|\frac{\partial}{\partial t} \Gamma\right| \leq C|\nabla \mathrm{Rc}|+2 \bar{\alpha}|\nabla \phi|\left|\nabla^{2} \phi\right|
$$

which yields a bound for $|\Gamma|$ by integration. Together with the bounds for $|\phi|$ and $|\nabla \&|$ that we obtain from (6.28), we conclude that $\left|\frac{\partial}{\partial t} \partial g\right|_{\delta}$ is uniformly bounded, and hence - again by integration $-|\partial g|_{\delta}$ is uniformly bounded on $U \times[0, T)$. Finally, using a partition of unity for the chosen atlas, we obtain a uniform bound for $|\tilde{\nabla} g|_{\tilde{g}}$ on $M \times[0, T)$. A short computation as for (6.30) yields

$$
\left|\frac{\partial}{\partial t} \tilde{\nabla}^{k} g\right|_{\tilde{g}} \leq C\left|\frac{\partial}{\partial t} \tilde{\nabla}^{k} g\right| \leq \sum_{i=0}^{k} c_{i}|\Gamma|^{i}\left|\nabla^{k-i} \phi\right|+\sum_{i=1}^{k-1} c_{i}^{\prime}\left|\partial^{i} \Gamma\right|\left|\tilde{\nabla}^{k-1-i} \phi\right|,
$$

where the constants $c_{i}, c_{i}^{\prime}$ only depend on $m$ and $k$. From this formula, we inductively obtain the desired bounds for $\left|\tilde{\nabla}^{k} g\right|_{\tilde{g}}$. Similarly, the estimates for $\left|\tilde{\nabla}^{k} \operatorname{Rm}(x, t)\right|_{\tilde{g}}$ and $\left|\tilde{\nabla}^{k} \phi(x, t)\right|_{\tilde{g}}$ are obtained from (6.28) with a transformation analogous to (6.31). The lemma then follows by plugging everything together.

Finally, we obtain our desired criterion for long-time existence.

Theorem 6.16. - Let $(g(t), \phi(t))_{t \in[0, T)}$ solve $(R H)_{\alpha}$ with non-increasing $\alpha(t) \in[\underline{\alpha}, \bar{\alpha}]$, $0<\underline{\alpha} \leq \bar{\alpha}<\infty$ and $T<\infty$. Suppose that $T<\infty$ is maximally chosen, i.e., the solution cannot be extended beyond $T$ in a smooth way. Then the curvature of $(M, g(t))$ has to become unbounded for $t \nearrow T$ in the sense that

$$
\limsup _{t \nearrow T}\left(\max _{x \in M}|\operatorname{Rm}(x, t)|^{2}\right)=\infty .
$$


Proof. - The proof is by contradiction. Suppose that the curvature stays bounded on $[0, T)$, say $|\mathrm{Rm}| \leq R_{0}$. For any point $x \in M$ and vector $X \in T_{x} M$, define

$$
g(x, T)(X, X):=\lim _{t \rightarrow T} g(x, t)(X, X) .
$$

We estimate

$$
|g(x, T)(X, X)-g(x, t)(X, X)| \leq \int_{t}^{T} 2|\phi(x, \tau)(X, X)| d \tau \leq C|X|^{2}(T-t),
$$

where we used again the fact that $\phi$ is uniformly bounded on $M \times[0, T)$. This shows that the limit $g(x, T)(X, X)$ is well defined and continuous in $x$. Hence, we obtain a continuous limit $g(\cdot, T) \in \Gamma\left(\operatorname{Sym}^{2}\left(T^{*} M\right)\right)$ by polarization. From [6, Lemma 6.49], all metrics $g(\cdot, t)$ are uniformly equivalent - as in (6.29) - which implies that this limit must be a (continuous) Riemannian metric. Moreover, we define $\phi(x, T):=\lim _{t \rightarrow T} \phi(x, t)$, where we use again the embedding $e_{N}: N \hookrightarrow \mathbb{R}^{d}$ to interpret $\phi$ as a map into $\mathbb{R}^{d}$. We estimate

$$
|\phi(x, T)-\phi(x, t)| \leq \int_{t}^{T}\left|\frac{\partial}{\partial t} \phi(x, \tau)\right| d \tau \leq C(T-t),
$$

since the bound on $\left|\nabla^{2} \phi\right|$ yields a bound on $\left|\frac{\partial}{\partial t} \phi\right|=\left|\tau_{g} \phi\right|$. This implies that $\phi(\cdot, T)$ is well defined and continuous in $x$. The uniform bounds (6.27) from Lemma 6.15 then also hold for the limit $(g(T), \phi(T))$ and hence $g(T)$ and $\phi(T)$ are smooth. Indeed, for an arbitrary background metric $\tilde{g}$, we have

$$
\left|\tilde{\nabla}^{k} g(T)-\tilde{\nabla}^{k} g(t)\right|_{\tilde{g}} \leq \int_{t}^{T}\left|\frac{\partial}{\partial t} \tilde{\nabla}^{k} g(\tau)\right|_{\tilde{g}} d \tau \leq C(T-t),
$$

which follows from the uniform bound for $\left|\frac{\partial}{\partial t} \tilde{\nabla} g\right|_{\tilde{g}}$ that we have derived in the lemma above. This means, the convergence $g(t) \rightarrow g(T)$ is smooth. With

$$
\left|\tilde{\nabla}^{k} \phi(T)-\tilde{\nabla}^{k} \phi(t)\right|_{\tilde{g}} \leq \int_{t}^{T}\left|\frac{\partial}{\partial t} \tilde{\nabla}^{k} \phi(\tau)\right|_{\tilde{g}} d \tau \leq C(T-t)
$$

we see that also $\phi(t) \rightarrow \phi(T)$ uniformly in any $C^{k}$-norm. Finally, restarting the flow with $(g(T), \phi(T))$ as new initial data, we obtain a solution $(g(t), \phi(t))_{t \in[T, T+\varepsilon)}$ by the short-time existence result from Chapter 2. This yields an extension of our solution beyond time $T$ which is smooth in space for each time. From the flow equations and the uniform bounds on $\left|\nabla^{k} \mathrm{Rm}\right|$ as well as $\left|\nabla^{k} \phi\right|$, the time derivatives (and hence also the mixed derivatives) are smooth too, in particular near $t=T$. This means, the extension of the flow is smooth in space and time, contradicting the maximality of $T$.

\section{Monotonicity formula and no breathers theorem}

The entropy functional $W_{\alpha}$ introduced in this section is the analogue of Perelman's shrinker entropy for the Ricci flow from [31, Section 3]. It is obtained from the energy functional $\mathcal{F}_{\alpha}$ from (3.1), by introducing a positive scale factor $\tau$ (later interpreted as a backwards time) and some correction terms. For detailed explanations of Perelman's result, we again refer to Chow et al. [4, Chapter 6] and Müller [27, Chapter 3]. Moreover for the special case $N \subseteq \mathbb{R}$, the entropy functional $W_{\alpha}$ can be found in List's dissertation [22]. 


\subsection{The entropy functional and its first variation}

Let again $g=g_{i j} \in \Gamma\left(\operatorname{Sym}_{+}^{2}\left(T^{*} M\right)\right), \phi \in C^{\infty}(M, N), f: M \rightarrow \mathbb{R}$ and $\tau>0$. For a time-independent coupling constant $\alpha(t) \equiv \alpha>0$, we set

$$
W_{\alpha}(g, \phi, f, \tau):=\int_{M}\left(\tau\left(R_{g}+|\nabla f|_{g}^{2}-\alpha|\nabla \phi|_{g}^{2}\right)+f-m\right)(4 \pi \tau)^{-m / 2} e^{-f} d V_{g} .
$$

As in Section 3, we take variations $g^{\varepsilon}=g+\varepsilon h, f^{\varepsilon}=f+\varepsilon \ell, \phi^{\varepsilon}=\pi_{N}(\phi+\varepsilon \vartheta)$, such that $\delta g=h, \delta f=\ell$ and $\delta \phi=\vartheta$. Additionally, set $\tau^{\varepsilon}=\tau+\varepsilon \sigma$ for some $\sigma \in \mathbb{R}$, i.e., $\delta \tau=\sigma$. The variation

$$
\delta W_{\alpha}:=\delta W_{\alpha, g, \phi, f, \tau}(h, \vartheta, \ell, \sigma):=\left.\frac{d}{d \varepsilon}\right|_{\varepsilon=0} W_{\alpha}\left(g+\varepsilon h, \pi_{N}(\phi+\varepsilon \vartheta), f+\varepsilon \ell, \tau+\varepsilon \sigma\right)
$$

is easiest to compute using the variation of $\mathcal{F}_{\alpha}$ and

$$
W_{\alpha}(g, \phi, f, \tau)=(4 \pi \tau)^{-m / 2}\left(\tau \mathcal{F}_{\alpha}(g, \phi, f)+\int_{M}(f-m) e^{-f} d V\right) .
$$

Using

$$
\delta \int_{M}(f-m) e^{-f} d V=\int_{M}\left(\ell+(f-m)\left(\frac{1}{2} \operatorname{tr}_{g} h-\ell\right)\right) e^{-f} d V
$$

and Equation (3.2) for the variation of $\mathcal{F}_{\alpha}(g, \phi, f)$, we get from (7.2)

$$
\begin{aligned}
\delta W_{\alpha}= & \int_{M}-\tau h_{i j}\left(R_{i j}+\nabla_{i} \nabla_{j} f-\alpha \nabla_{i} \phi \nabla_{j} \phi\right) d \mu \\
& +\int_{M} \tau\left(\frac{1}{2} \operatorname{tr}_{g} h-\ell\right)\left(2 \triangle f-|\nabla f|^{2}+R-\alpha|\nabla \phi|^{2}+\frac{f-m}{\tau}\right) d \mu \\
& +\int_{M}\left(\ell+\sigma\left(1-\frac{m}{2}\right)\left(R+|\nabla f|^{2}-\alpha|\nabla \phi|^{2}\right)-\frac{m \sigma}{2 \tau}(f-m)\right) d \mu \\
& +\int_{M} 2 \tau \alpha \vartheta\left(\tau_{g} \phi-\langle\nabla \phi, \nabla f\rangle\right) d \mu,
\end{aligned}
$$

where we used the abbreviation $d \mu:=(4 \pi \tau)^{-m / 2} e^{-f} d V$. Rearranging the terms, writing

$$
\ell=\left(-\tau h_{i j}+\sigma g_{i j}\right)\left(\frac{-1}{2 \tau} g_{i j}\right)+\tau\left(\frac{1}{2} \operatorname{tr}_{g} h-\ell-\frac{m \sigma}{2 \tau}\right)\left(\frac{-1}{\tau}\right),
$$

and using $\int_{M}\left(\triangle f-|\nabla f|^{2}\right) d \mu=-(4 \pi \tau)^{-m / 2} \int_{M} \triangle\left(e^{-f}\right) d V=0$, we get

$$
\begin{aligned}
\delta W_{\alpha}= & \int_{M}\left(-\tau h_{i j}+\sigma g_{i j}\right)\left(R_{i j}+\nabla_{i} \nabla_{j} f-\alpha \nabla_{i} \phi \nabla_{j} \phi-\frac{1}{2 \tau} g_{i j}\right) d \mu \\
& +\int_{M} \tau\left(\frac{1}{2} \operatorname{tr}_{g} h-\ell-\frac{m \sigma}{2 \tau}\right)\left(2 \triangle f-|\nabla f|^{2}+R-\alpha|\nabla \phi|^{2}+\frac{f-m-1}{\tau}\right) d \mu \\
& +\int_{M} 2 \tau \alpha \vartheta\left(\tau_{g} \phi-\langle\nabla \phi, \nabla f\rangle\right) d \mu .
\end{aligned}
$$

\subsection{Fixing the background measure}

Similar to Section 3, we now fix the measure $d \mu=(4 \pi \tau)^{-m / 2} e^{-f} d V$. This means, we have $f=-\log \left((4 \pi \tau)^{m / 2} \frac{d \mu}{d V}\right)$ and from $0=\delta d \mu=\left(\frac{1}{2} \operatorname{tr}_{g} h-\ell-\frac{m \sigma}{2 \tau}\right) d \mu$, we deduce $\ell=\frac{1}{2} \operatorname{tr}_{g} h-\frac{m \sigma}{2 \tau}$. Moreover, we require the variation of $\tau$ to satisfy $\delta \tau=\sigma=-1$. This allows us to interpret $\tau$ as backwards time later. We then write

$$
W_{\alpha}^{\mu}(g, \phi, \tau):=W_{\alpha}\left(g, \phi,-\log \left((4 \pi \tau)^{m / 2} \frac{d \mu}{d V}\right), \tau\right)
$$


and

$$
\delta W_{\alpha, g, \phi, \tau}^{\mu}(h, \vartheta):=\delta W_{\alpha, g, \phi,-\log \left((4 \pi \tau)^{m / 2} \frac{d \mu}{d V}\right), \tau}\left(h, \vartheta, \frac{1}{2} \operatorname{tr}_{g} h+\frac{m}{2 \tau},-1\right) .
$$

The variation formula above reduces to

$$
\begin{aligned}
\delta W_{\alpha, g, \phi, \tau}^{\mu}(h, \vartheta)= & \int_{M}\left(-\tau h_{i j}-g_{i j}\right)\left(R_{i j}+\nabla_{i} \nabla_{j} f-\alpha \nabla_{i} \phi \nabla_{j} \phi-\frac{1}{2 \tau} g_{i j}\right) d \mu \\
& +\int_{M} 2 \tau \alpha \vartheta\left(\tau_{g} \phi-\langle\nabla \phi, \nabla f\rangle\right) d \mu,
\end{aligned}
$$

which is monotone under the gradient-like system of evolution equations given by

$$
\left\{\begin{aligned}
\frac{\partial}{\partial t} g_{i j} & =-2\left(R_{i j}+\nabla_{i} \nabla_{j} f-\alpha \nabla_{i} \phi \nabla_{j} \phi\right), \\
\frac{\partial}{\partial t} \phi & =\tau_{g} \phi-\langle\nabla \phi, \nabla f\rangle, \\
\frac{\partial}{\partial t} f & =-R-\Delta f+\alpha|\nabla \phi|^{2}+\frac{m}{2 \tau} \\
\frac{\partial}{\partial t} \tau & =-1 .
\end{aligned}\right.
$$

As in Section 3, pulling back the solutions of (7.5) with the family of diffeomorphisms generated by $\nabla f$, we get a solution of

$$
\left\{\begin{aligned}
\frac{\partial}{\partial t} g & =-2 \mathrm{Rc}+2 \alpha \nabla \phi \otimes \nabla \phi \\
\frac{\partial}{\partial t} \phi & =\tau_{g} \phi \\
\frac{\partial}{\partial t} \tau & =-1 \\
0 & =\square^{*}\left((4 \pi \tau)^{-m / 2} e^{-f}\right) .
\end{aligned}\right.
$$

Since $W_{\alpha}$ is diffeomorphism invariant, we find the analogue to Proposition 3.1.

Proposition 7.1. - Let $(g(t), \phi(t))_{t \in[0, T)}$ be a solution of $(R H)_{\alpha}$ with $\alpha(t) \equiv \alpha>0$, $\tau$ a backwards time with $\frac{\partial}{\partial t} \tau=-1$ and $(4 \pi \tau)^{-m / 2} e^{-f}$ a solution of the adjoint heat equation under the flow. Then the entropy functional $W_{\alpha}(g, \phi, f, \tau)$ is non-decreasing with

$$
\begin{aligned}
\frac{d}{d t} W_{\alpha}= & \int_{M} 2 \tau\left|\operatorname{Rc}-\alpha \nabla \phi \otimes \nabla \phi+\operatorname{Hess}(f)-\frac{g}{2 \tau}\right|^{2}(4 \pi \tau)^{-m / 2} e^{-f} d V \\
& +\int_{M} 2 \tau \alpha\left|\tau_{g} \phi-\langle\nabla \phi, \nabla f\rangle\right|^{2}(4 \pi \tau)^{-m / 2} e^{-f} d V .
\end{aligned}
$$

Remark 7.2. - As seen in Corollary 3.2 for the energy $\mathcal{F}_{\alpha}$, the monotonicity of the entropy $W_{\alpha}$ also holds true for non-increasing positive coupling functions $\alpha(t)$ instead of a constant $\alpha>0$.

\subsection{Minimizing over all probability measures}

Similar to $\lambda_{\alpha}(g, \phi)$ defined in (3.9), we set

$$
\begin{aligned}
\mu_{\alpha}(g, \phi, \tau): & =\inf \left\{W_{\alpha}^{\mu}(g, \phi, \tau) \mid \mu(M)=1\right\} \\
& =\inf \left\{W_{\alpha}(g, \phi, f, \tau) \mid \int_{M}(4 \pi \tau)^{-m / 2} e^{-f} d V=1\right\} .
\end{aligned}
$$

Our goal is again to show that the infimum is always achieved. Note that for $\tilde{g}=\tau g$ we have $R_{\tilde{g}}=\frac{1}{\tau} R_{g},|\nabla f|_{\tilde{g}}^{2}=\frac{1}{\tau}|\nabla f|_{g}^{2},|\nabla \phi|_{\tilde{g}}^{2}=\frac{1}{\tau}|\nabla \phi|_{g}^{2}, d V_{\tilde{g}}=\tau^{m / 2} d V_{g}$ and thus

$$
\mu_{\alpha}(\tau g, \phi, \tau)=\mu_{\alpha}(g, \phi, 1) \text {. }
$$


We can hence reduce the problem to the special case where $\tau=1$. Set $v=(4 \pi)^{-m / 4} e^{-f / 2}$. This yields

$$
W_{\alpha}(g, \phi, v, 1)=\int_{M} v\left(R v-4 \triangle v-\alpha|\nabla \phi|^{2} v-2 v \log v-\frac{m v}{2} \log (4 \pi)-m v\right) d V,
$$

and hence $\mu_{\alpha}(g, \phi, 1)=\inf \left\{W_{\alpha}(g, \phi, v, 1) \mid \int_{M} v^{2} d V=1\right\}$ is the smallest eigenvalue of $L(v)=-4 \triangle v+\left(R-\alpha|\nabla \phi|^{2}-\frac{m}{2} \log (4 \pi)-m\right) v-2 v \log v$ and $v$ is a corresponding normalized eigenvector. As in Section 3, a unique smooth positive normalized eigenvector $v_{\text {min }}$ exists (cf. Rothaus [36] or List [22]) and we get the following.

Proposition 7.3. - Let $(g(t), \phi(t))$ solve $(R H)_{\alpha}$ for a constant $\alpha>0$ and let $\frac{\partial}{\partial t} \tau=-1$. Then $\mu_{\alpha}(g, \phi, \tau)$ is monotone non-decreasing in time. Moreover, it is constant if and only if

$$
\left\{\begin{array}{l}
0=\mathrm{Rc}-\alpha \nabla \phi \otimes \nabla \phi+\operatorname{Hess}(f)-\frac{g}{2 \tau}, \\
0=\tau_{g} \phi-\langle\nabla \phi, \nabla f\rangle,
\end{array}\right.
$$

for the minimizer $f$ that corresponds to $v_{\min }$. As always, the monotonicity result stays true if we allow $\alpha(t)$ to be a positive non-increasing function instead of a constant.

Proof. - The proof is completely analogous to the proof of Proposition 3.3, using the monotonicity of $W_{\alpha}$ instead of the monotonicity of $\mathcal{F}_{\alpha}$.

\subsection{Non-existence of nontrivial breathers}

Breathers correspond to periodic solutions modulo diffeomorphisms and scaling, generalizing the notion of solitons defined in Definition 2.1.

Definition 7.4. - A solution $(g(t), \phi(t))_{t \in[0, T)}$ of $(R H)_{\alpha}$ is called a breather if there exist $t_{1}, t_{2} \in[0, T), t_{1}<t_{2}$, a diffeomorphism $\psi: M \rightarrow M$ and a constant $c \in \mathbb{R}_{+}$such that

$$
\left\{\begin{array}{l}
g\left(t_{2}\right)=c \psi^{*} g\left(t_{1}\right) \\
\phi\left(t_{2}\right)=\psi^{*} \phi\left(t_{1}\right) .
\end{array}\right.
$$

The cases $c<1, c=1$ and $c>1$ correspond to shrinking, steady and expanding breathers.

Theorem 7.5. - Let $M$ and $N$ be closed and let $(g(t), \phi(t))_{t \in[0, T)}$ be a solution of $(R H)_{\alpha}$ with $\alpha(t) \equiv \alpha$.

i) If this solution is a steady breather, then it necessarily is a gradient steady soliton. Moreover, $\phi(t)$ is harmonic and $\mathrm{Rc}=\alpha \nabla \phi \otimes \nabla \phi$, i.e., the solution is stationary.

ii) If the solution is an expanding breather, then it necessarily is a gradient expanding soliton. Again $\phi(t)$ must be harmonic (and thus stationary, $\phi(t)=\phi(0)$ ), while $g(t)$ changes only by scaling.

iii) If the solution is a shrinking breather, then it has to be a gradient shrinking soliton.

If we assume in addition that $\operatorname{dim} M=2$ or that $(M, g(0))$ is Einstein, then in the first two cases above, $\phi(t)$ is not only harmonic but also conformal, hence a minimal branched immersion, provided that it is non-constant.

$4^{\text {e }}$ SÉRIE - TOME $45-2012-$ NNo $^{1}$ 
Proof. - This is an application of the monotonicity results for $\lambda_{\alpha}(g, \phi)$ from Proposition 3.3 and for $\mu_{\alpha}(g, \phi, \tau)$ from Proposition 7.3. Since the proof is very similar to the Ricci flow case solved by Perelman in [31], we closely follow the notes from Kleiner and Lott [19] on Perelman's paper.

i) Assume $(g(t), \phi(t))_{t \in[0, T)}$ is a steady breather. Then there exist two times $t_{1}, t_{2}$ such that (7.10) holds with $c=1$. From diffeomorphism invariance of $\lambda_{\alpha}(g, \phi)$ defined in (3.9), we obtain $\lambda_{\alpha}(g, \phi)\left(t_{1}\right)=\lambda_{\alpha}(g, \phi)\left(t_{2}\right)$. From Proposition 3.3, we get condition (3.10) on $\left[t_{1}, t_{2}\right]$, which means that $(g(t), \phi(t))$ must be a gradient steady soliton according to Lemma 2.2 and uniqueness of solutions. Moreover, the minimizer $f=-2 \log v_{\min }$ which realizes $\lambda_{\alpha}(g, \phi)$ is the soliton potential. From $\left(-4 \triangle+R-\alpha|\nabla \phi|^{2}\right) v_{\min }=\lambda_{\alpha}(g, \phi) v_{\min }=: \lambda_{\alpha} v_{\min }$, we obtain

$$
2 \triangle f-|\nabla f|^{2}+R-\alpha|\nabla \phi|^{2}=\lambda_{\alpha} .
$$

Since $(g(t), \phi(t))$ is a steady soliton, (2.5) holds with $\sigma=0$. Plugging this into (7.11) yields $\triangle f-|\nabla f|^{2}=\lambda_{\alpha}$, and we obtain from $\int_{M} e^{-f} d V=1$

$$
\lambda_{\alpha}=\int_{M} \lambda_{\alpha} e^{-f} d V=\int_{M}\left(\triangle f-|\nabla f|^{2}\right) e^{-f} d V=-\int_{M} \triangle\left(e^{-f}\right) d V=0,
$$

i.e., $\triangle f=|\nabla f|^{2}$. Another integration yields

$$
\int_{M}|\nabla f|^{2} d V=\int_{M} \triangle f d V=0
$$

and thus $\nabla f \equiv 0, \operatorname{Hess}(f) \equiv 0$ on $M \times[0, T)$ and (3.10) becomes

$$
\left\{\begin{array}{l}
0=\mathrm{Rc}-\alpha \nabla \phi \otimes \nabla \phi \\
0=\tau_{g} \phi
\end{array}\right.
$$

In particular, $\phi(t)$ is harmonic and $(g(t), \phi(t))$ is stationary.

ii) The proof here is analogous to the case of steady breathers, but we first need to construct a scaling invariant version of $\lambda_{\alpha}(g, \phi)$. We define

$$
\bar{\lambda}_{\alpha}(g, \phi):=\lambda_{\alpha}(g, \phi)\left(\int_{M} d V_{g}\right)^{2 / m} .
$$

This quantity is invariant under rescaling $\tilde{g}=c g$. A proof of this fact is given in the appendix of the author's dissertation [28]. Moreover, we claim that at times where $\bar{\lambda}_{\alpha}(t):=\bar{\lambda}_{\alpha}(g, \phi)(t) \leq 0$, we have $\frac{\partial}{\partial t} \bar{\lambda}_{\alpha}(t) \geq 0$. Indeed, note that $\bar{f}=\log V(t)$ satisfies $\int_{M} e^{-\bar{f}} d V=1$ and is thus an admissible test function in the definition of $\lambda_{\alpha}$, hence

$$
\lambda_{\alpha}(g, \phi) \leq \mathcal{F}_{\alpha}(g, \phi, \log V(t))=\int_{M} S e^{-\log V(t)} d V=V(t)^{-1} \int_{M} S d V .
$$

With the assumption $\lambda_{\alpha}(t) \leq 0$, we find

$$
\begin{aligned}
\frac{\partial}{\partial t} \bar{\lambda}_{\alpha} \geq & 2 V^{2 / m} \int_{M}\left(\left|\varnothing+\operatorname{Hess}(f)-\frac{1}{m}(S+\triangle f) g\right|^{2}+\alpha\left|\tau_{g} \phi-\langle\nabla \phi, \nabla f\rangle\right|^{2}\right) d \mu \\
& +\frac{2}{m} V^{2 / m}\left(\int_{M}(S+\triangle f)^{2} d \mu-\left(\int_{M}(S+\triangle f) d \mu\right)^{2}\right),
\end{aligned}
$$

the right hand side being nonnegative by Hölder's inequality (see again [28] for a more detailed computation). Now, assume that $(g(t), \phi(t))$ is an expanding breather. Since 
$\bar{\lambda}_{\alpha}(g, \phi)$ is invariant under diffeomorphisms and scaling, we have $\bar{\lambda}_{\alpha}\left(t_{1}\right)=\bar{\lambda}_{\alpha}\left(t_{2}\right)$ for the two times $t_{1}, t_{2}$ that satisfy (7.10). Since $V\left(t_{1}\right)<V\left(t_{2}\right)$, there must be a time $t_{0} \in\left[t_{1}, t_{2}\right]$ with $\frac{\partial}{\partial t} V\left(t_{0}\right)>0$ and hence with (7.13)

$$
\lambda_{\alpha}\left(t_{0}\right) \leq V\left(t_{0}\right)^{-1} \int_{M} S d V=-V\left(t_{0}\right)^{-1} \frac{\partial}{\partial t} V\left(t_{0}\right)<0 .
$$

The claim applies and we obtain $\bar{\lambda}_{\alpha}\left(t_{1}\right) \leq \bar{\lambda}_{\alpha}\left(t_{0}\right)<0$ and since $\bar{\lambda}_{\alpha}\left(t_{2}\right)=\bar{\lambda}_{\alpha}\left(t_{1}\right)$, we see that $\bar{\lambda}_{\alpha}(t)$ must be a negative constant. Hence, both lines on the right hand side of (7.14) have to vanish. This means that $(S+\triangle f)$ has to be constant in space for all $t$ and because $\lambda_{\alpha}(t)=\int_{M}(S+\triangle f) d \mu$, this constant has to be $\lambda_{\alpha}(t)$. From the first line of (7.14) we obtain

$$
\left\{\begin{array}{l}
0=\mathrm{Rc}-\alpha \nabla \phi \otimes \nabla \phi+\operatorname{Hess}(f)-\frac{\lambda_{\alpha}}{m} g \\
0=\tau_{g} \phi-\langle\nabla \phi, \nabla f\rangle .
\end{array}\right.
$$

By Lemma 2.2, $(g(t), \phi(t))_{t \in[0, T)}$ is an expanding soliton with potential $f=-2 \log v_{\min }$. This means that we can use (7.11), which implies

$$
0=2 \triangle f-|\nabla f|+S-\lambda_{\alpha}=2 \triangle f-|\nabla f|+S-(\triangle f+S)=\triangle f-|\nabla f|
$$

and thus by integration $\nabla f \equiv 0, \operatorname{Hess}(f) \equiv 0$, as above. Plugging this into (7.15), the second equation tells us that $\phi(t)$ is harmonic and the first equation yields

$$
\frac{\partial}{\partial t} g=-2 \mathrm{Rc}+2 \alpha \nabla \phi \otimes \nabla \phi=-2 \frac{\lambda_{\alpha}}{m} g,
$$

i.e., $(M, g(t))$ simply expands without changing its shape.

iii) If $(g(t), \phi(t))$ is a shrinking breather, there exist $t_{1}, t_{2}$ and $c<1$ such that (7.10) is satisfied. We define

$$
\tau_{0}:=\frac{t_{2}-c t_{1}}{1-c}>t_{2}, \quad \text { and } \quad \tau(t)=\tau_{0}-t .
$$

Note that $\tau(t)$ is always positive on $\left[t_{1}, t_{2}\right]$. Moreover, $c=\left(\tau_{0}-t_{2}\right) /\left(\tau_{0}-t_{1}\right)=$ $\tau\left(t_{2}\right) / \tau\left(t_{1}\right)$. Then, from the scaling behavior of $\mu_{\alpha}(g, \phi, \tau)$ and diffeomorphism invariance we obtain

$$
\begin{aligned}
\mu_{\alpha}\left(g\left(t_{2}\right), \phi\left(t_{2}\right), \tau\left(t_{2}\right)\right) & =\mu_{\alpha}\left(c \psi^{*} g\left(t_{1}\right), \psi^{*} \phi\left(t_{1}\right), c \tau\left(t_{1}\right)\right) \\
& =\mu_{\alpha}\left(\psi^{*} g\left(t_{1}\right), \psi^{*} \phi\left(t_{1}\right), \tau\left(t_{1}\right)\right) \\
& =\mu_{\alpha}\left(g\left(t_{1}\right), \phi\left(t_{1}\right), \tau\left(t_{1}\right)\right) .
\end{aligned}
$$

By the equality case of the monotonicity result in Proposition 7.3, $(g(t), \phi(t))$ must satisfy (7.9) and according to Lemma 2.2 thus has to be a gradient shrinking soliton.

It remains to prove the additional statement in the cases where $\operatorname{dim} M=2$ or $(M, g(0))$ is Einstein. If $(g(t), \phi(t))$ is a steady or expanding breather, we have seen that $\frac{\partial}{\partial t} g=c g$. In particular, if $(M, g(t))$ is Einstein at $t=0$ it remains Einstein under the flow. Moreover, since $\mathrm{Rc}=\frac{R}{m} g$ in these two cases, we get

$$
\left(\phi^{*} \gamma\right)_{i j}=\nabla_{i} \phi \nabla_{j} \phi=\frac{1}{2 \alpha}\left(\frac{\partial}{\partial t} g_{i j}+2 R_{i j}\right)=\frac{1}{2 \alpha}\left(2 \frac{R}{m}+c\right) g_{i j},
$$

i.e., $\phi$ is conformal. It is a well-known fact that conformal harmonic maps have to be minimal branched immersions (cf. Hartman-Wintner [17]). 


\section{Reduced volume and non-collapsing theorem}

Let us briefly restate the main result from our previous article [29] about the monotonicity of reduced volumes for flows of the form $\frac{\partial}{\partial t} g_{i j}=-2 S_{i j}$, where $S_{i j}$ is a symmetric tensor with trace $S=g{ }^{i j} S_{i j}$. (The results can also be found in the author's thesis [28].)

\subsection{Monotonicity of backwards reduced volume}

In order to define the backwards reduced distance and volume, we need a backwards time $\tau(t)$ with $\frac{\partial}{\partial t} \tau(t)=-1$. Without loss of generality, one may assume (possibly after a time shift) that $\tau=-t$.

Definition 8.1. - Assume $\frac{\partial}{\partial \tau} g_{i j}=2 S_{i j}$ has a solution for $\tau \in[0, \bar{\tau}]$ and $0 \leq \tau_{1}<\tau_{2} \leq \bar{\tau}$, we define the $\mathscr{L}_{b}$-length of a curve $\eta:\left[\tau_{0}, \tau_{1}\right] \rightarrow M$ by

$$
\mathscr{L}_{b}(\eta):=\int_{\tau_{0}}^{\tau_{1}} \sqrt{\tau}\left(S(\eta(\tau))+\left|\frac{d}{d \tau} \eta(\tau)\right|^{2}\right) d \tau
$$

Fix the point $p \in M$ and $\tau_{0}=0$ and define the backwards reduced distance by

$$
\ell_{b}\left(q, \tau_{1}\right):=\inf _{\eta \in \Gamma}\left\{\frac{1}{2 \sqrt{\tau_{1}}} \int_{0}^{\tau_{1}} \sqrt{\tau}\left(S+\left|\frac{d}{d \tau} \eta\right|^{2}\right) d \tau\right\}
$$

where $\Gamma=\left\{\eta:\left[0, \tau_{1}\right] \rightarrow M \mid \eta(0)=p, \eta\left(\tau_{1}\right)=q\right\}$. The backwards reduced volume is defined by

$$
\tilde{V}_{b}(\tau):=\int_{M}(4 \pi \tau)^{-m / 2} e^{-\ell_{b}(q, \tau)} d V(q)
$$

The following is proved in [29, Theorem 1.4].

Theorem 8.2. - Suppose that $g(t)$ evolves by $\frac{\partial}{\partial t} g_{i j}=-2 S_{i j}$ and the quantity

$$
\begin{aligned}
\mathscr{D}(\&, X):= & \frac{\partial}{\partial t} S-\triangle S-2\left|S_{i j}\right|^{2}+4\left(\nabla_{i} S_{i j}\right) X_{j}-2\left(\nabla_{j} S\right) X_{j} \\
& +2 R_{i j} X_{i} X_{j}-2 S_{i j} X_{i} X_{j},
\end{aligned}
$$

is nonnegative for all vector fields $X \in \Gamma(T M)$ and all times $t$ for which the flow exists. Then the backwards reduced volume $\tilde{V}_{b}(\tau)$ is non-increasing in $\tau$, i.e., non-decreasing in $t$.

In our case where $S_{i j}$ is given by $R_{i j}-\alpha \nabla_{i} \phi \nabla_{j} \phi$, the evolution equation (4.14) for $S_{i j}$ together with $4\left(\nabla_{i} S_{i j}\right) X_{j}-2\left(\nabla_{j} S\right) X_{j}=-4 \alpha \tau_{g} \phi \nabla_{j} \phi X_{j}$ yields

$$
\mathscr{D}\left(S_{i j}, X\right)=2 \alpha\left|\tau_{g} \phi-\nabla_{X} \phi\right|^{2}-\dot{\alpha}|\nabla \phi|^{2}
$$

for all $X$ on $M$. This means, $\mathscr{D}\left(S_{i j}, X\right) \geq 0$ is satisfied for the $(R H)_{\alpha}$ flow with a positive non-increasing coupling function $\alpha(t)$ and the monotonicity of the reduced volume holds. 


\subsection{No local collapsing theorem}

We have seen in Section 6 that the metrics $g(t)$ along the $(R H)_{\alpha}$ flow are uniformly equivalent as long as the curvature on $M$ stays uniformly bounded. But a-priori, it could happen that at a singularity (i.e., when Rm blows up) the solution collapses geometrically in the following sense.

Definition 8.3. - Let $(g(t), \phi(t))_{t \in[0, T)}$ be a maximal solution of $(R H)_{\alpha}$, or more generally of any flow of the form $\frac{\partial}{\partial t} g_{i j}=-2 S_{i j}$. We say that this solution is locally collapsing at time $T$, if there is a sequence of times $t_{k} \nearrow T$ and a sequence of balls $B_{k}:=B_{g\left(t_{k}\right)}\left(x_{k}, r_{k}\right)$ at time $t_{k}$, such that the following holds. The ratio $r_{k}^{2} / t_{k}$ is bounded, the curvature satisfies $|\mathrm{Rm}| \leq r_{k}^{-2}$ on the parabolic neighborhood $B_{k} \times\left[t_{k}-r_{k}^{2}, t_{k}\right]$ and $r_{k}^{-m} \operatorname{vol}\left(B_{k}\right) \rightarrow 0$ as $k \rightarrow \infty$.

Using the monotonicity of the reduced volume, we obtain the following result.

THeOREM 8.4. - Let $(g(t), \phi(t))$ be a solution of $(R H)_{\alpha}$ with non-increasing $\alpha(t) \in[\underline{\alpha}, \bar{\alpha}]$, $0<\underline{\alpha} \leq \bar{\alpha}<\infty$ on a finite time interval $[0, T)$. Then this solution is not locally collapsing at $T$.

The only ingredients of the proof are the interior gradient estimates from Corollary 6.14 and the monotonicity of the backwards reduced volume stated above. Hence, every flow $\frac{\partial}{\partial t} g=-2 \varnothing$ that satisfies the assumption of Theorem 8.2 and some interior estimates for $\phi$, $\nabla S$ in the spirit of Corollary 6.14 will also satisfy the non-collapsing result. For the $(R H)_{\alpha}$ flow, it is possible to obtain a slightly stronger result using the monotonicity of $\mu_{\alpha}(g, \phi, \tau)$ from Section 7 instead of the monotonicity of the backwards reduced volume. In the special case $N \subseteq \mathbb{R}$, this can be found in List's dissertation [22, Section 7]. The proof in the case of $(R H)_{\alpha}$ is analogous. However, the result here is more general in the sense that it may be adopted to other flows $\frac{\partial}{\partial t} g=-2 \varnothing$ in the way explained above.

Proof. - The proof follows Perelman's results for the Ricci flow [31] very closely, see also the notes on his paper by Kleiner and Lott [19] and the book by Morgan and Tian [25]. However, we need the more general results from [29] that also hold for our coupled flow system. We only give a sketch.

The proof is by contradiction. Assume that there is some sequence of times $t_{k} \nearrow T$ and some sequence of balls $B_{k}:=B_{g\left(t_{k}\right)}\left(x_{k}, r_{k}\right)$ at each time $t_{k}$, such that $r_{k}^{2}$ is bounded, the curvature is bounded by $|\mathrm{Rm}| \leq r_{k}^{-2}$ on the parabolic neighborhood $B_{k} \times\left[t_{k}-r_{k}^{2}, t_{k}\right]$ and $r_{k}^{-m} \operatorname{vol}\left(B_{k}\right) \rightarrow 0$ as $k \rightarrow \infty$. Define $\varepsilon_{k}:=r_{k}^{-1} \operatorname{vol}\left(B_{k}\right)^{1 / m}$, then $\varepsilon_{k} \rightarrow 0$ for $k \rightarrow \infty$. For each $k$, we set $\tau_{k}(t)=t_{k}-t$ and define the backwards reduced volume $\tilde{V}_{k}$ using curves going backward in real time from the base point $\left(x_{k}, t_{k}\right)$, i.e., forward in time $\tau_{k}$ from $\tau_{k}=0$. The goal is to estimate the reduced volumes $\tilde{V}_{k}\left(\varepsilon_{k} r_{k}^{2}\right)$, where $\tau_{k}=\varepsilon_{k} r_{k}^{2}$ corresponds to the real time $t=t_{k}-\varepsilon_{k} r_{k}^{2}$, which is very close to $t_{k}$ and hence close to $T$. 
Claim 1. - We have $\lim _{k \rightarrow \infty} \tilde{V}_{k}\left(\varepsilon_{k} r_{k}^{2}\right)=0$.

Proof. - An $\mathscr{L}_{b}$-geodesic $\eta(\tau)$ starting at $\eta(0)=x_{k}$ is uniquely defined through its initial vector $v=\lim _{\tau \rightarrow 0} 2 \sqrt{\tau} X=\lim _{\lambda \rightarrow 0} \tilde{X}$. First, we show that if $|v| \leq \frac{1}{8} \varepsilon_{k}^{-1 / 2}$ with respect to the metric at $\left(x_{k}, t_{k}\right)$, then $\eta(\tau)$ does not escape from $B_{k}^{1 / 2}:=B_{g\left(t_{k}\right)}\left(x_{k}, r_{k} / 2\right)$ in time $\tau=\varepsilon_{k} r_{k}^{2}$. Write $\hat{t}_{k}=t_{k}-r_{k}^{2}$. Since $|\mathrm{Rm}| \leq r_{k}^{-2}$ on $B_{k} \times\left[\hat{t}_{k}, t_{k}\right]$ by assumption, we obtain from Corollary 6.14

$$
|\nabla \phi|^{2} \leq \frac{C}{t-\hat{t}_{k}}, \quad\left|\nabla^{2} \phi\right|^{2} \leq \frac{C}{\left(t-\hat{t}_{k}\right)^{2}}, \quad|\nabla \mathrm{Rm}|^{2} \leq \frac{C}{\left(t-\hat{t}_{k}\right)^{3}}, \quad \text { on } B_{k}^{1 / 2} \times\left(\hat{t}_{k}, t_{k}\right),
$$

for some constant $C$ independent of $k$. Without loss of generality, $\varepsilon_{k} \leq \frac{1}{2}$ so that $t-\hat{t}_{k} \geq \frac{1}{2} r_{k}^{2}$ whenever $t \in\left[t_{k}-\varepsilon_{k} r_{k}^{2}, t_{k}\right)$. This means that

$$
|\nabla \phi|^{2} \leq C r_{k}^{-2}, \quad\left|\nabla^{2} \phi\right| \leq C r_{k}^{-2}, \quad|\nabla \mathrm{Rm}| \leq C r_{k}^{-3}, \quad \text { on } B_{k}^{1 / 2} \times\left[t_{k}-\varepsilon_{k} r_{k}^{2}, t_{k}\right) .
$$

Together with the assumption $|\mathrm{Rm}| \leq r_{k}^{-2}$, this yields

$$
\begin{aligned}
|\phi| & \leq|\mathrm{Rc}|+|\nabla \phi|^{2} \leq C r_{k}^{-2}, \\
|\nabla S| & \leq|\nabla R|+|\nabla \phi|\left|\nabla^{2} \phi\right| \leq C r_{k}^{-3},
\end{aligned}
$$

on $B_{k}^{1 / 2} \times\left[t_{k}-\varepsilon_{k} r_{k}^{2}, t_{k}\right)$. Plugging this into the Estimate (4.5) of [29], we get

$$
\frac{\partial}{\partial \lambda}|\tilde{X}| \leq \lambda C|\tilde{X}| r_{k}^{-2}+\lambda^{2} C r_{k}^{-3} \leq C|\tilde{X}| \varepsilon_{k}^{1 / 2} r_{k}^{-1}+C \varepsilon_{k} r_{k}^{-1}
$$

for $\lambda=\sqrt{\tau} \leq \sqrt{\varepsilon_{k} r_{k}^{2}}=\varepsilon_{k}^{1 / 2} r_{k}$. Since $|\tilde{X}(0)|=|v| \leq \frac{1}{8} \varepsilon_{k}^{-1 / 2}$ we obtain the estimate $|\tilde{X}(\lambda)| \leq \frac{1}{4} \varepsilon_{k}^{-1 / 2}$ for all $\tau \in\left[0, \varepsilon_{k} r_{k}^{2}\right]$ if $k$ is large enough, i.e., $\varepsilon_{k}$ small enough. With an integration, we find

$$
\int_{0}^{\varepsilon_{k} r_{k}^{2}}|X(\tau)| d \tau=\int_{0}^{\sqrt{\varepsilon_{k}} r_{k}}|\tilde{X}(\lambda)| d \lambda \leq \int_{0}^{\sqrt{\varepsilon_{k}} r_{k}} \frac{1}{4} \varepsilon_{k}^{-1 / 2} d \lambda \leq \frac{1}{4} r_{k} .
$$

Since the metrics $g(\tau=0)$ and $g\left(\tau=\varepsilon_{k} r_{k}^{2}\right)$ are close to each other, the length of the curve $\eta$ measured with respect to $g(\tau=0)=g\left(t_{k}\right)$ will be at most $r_{k} / 2$ for large enough $k$. This means that indeed

$$
\left(\eta(\tau), t_{k}-\tau\right) \in B_{k}^{1 / 2} \times\left[t_{k}-\varepsilon_{k} r_{k}^{2}, t_{k}\right), \quad \forall 0<\tau \leq \varepsilon_{k} r_{k}^{2} .
$$

With the bounds from (8.4) and the lower bound in [29, Lemma 4.1], we obtain

$$
\mathscr{L}_{b}(\eta) \geq-C r_{k}^{-2}\left(\varepsilon_{k} r_{k}^{2}\right)^{3 / 2}=-C \varepsilon_{k}^{3 / 2} r_{k}, \quad \text { i.e. } \quad \ell_{b}\left(q, \varepsilon_{k} r_{k}^{2}\right) \geq-C \varepsilon_{k} .
$$

Thus, the contribution to the reduced volume $\tilde{V}_{k}\left(\varepsilon_{k} r_{k}^{2}\right)$ coming from $\mathscr{L}_{b}$-geodesics with initial vector $|v| \leq \frac{1}{8} \varepsilon_{k}^{-1 / 2}$ is bounded above for large $k$ by

$$
\int_{B_{k}^{1 / 2}}\left(4 \pi \varepsilon_{k} r_{k}^{2}\right)^{-m / 2} e^{C \varepsilon_{k}} d V \leq C \varepsilon_{k}^{-m / 2} r_{k}^{-m} \operatorname{vol}\left(B_{k}^{1 / 2}\right) \leq C \varepsilon_{k}^{m / 2} \rightarrow 0 \quad(k \rightarrow \infty) .
$$

Next, we estimate the contribution of geodesics with large initial vector $|v|>\frac{1}{8} \varepsilon_{k}^{-1 / 2}$ to the reduced volume $\tilde{V}_{k}\left(\varepsilon_{k} r_{k}^{2}\right)$. Note that we can write the reduced volume with base point $\left(x_{k}, t_{k}\right)$ as

$\tilde{V}_{k}\left(\tau_{1}\right)=\int_{M}\left(4 \pi \tau_{1}\right)^{-m / 2} e^{-\ell_{b}\left(q, \tau_{1}\right)} d V(q)=\int_{\Omega\left(\tau_{1}, k\right)}\left(4 \pi \tau_{1}\right)^{-m / 2} e^{-\ell_{b}\left(\mathscr{L}_{b} \exp _{x_{k}}^{\tau_{1}}(v), \tau_{1}\right)} J\left(v, \tau_{1}\right) d v$. 
Here, $\mathscr{L}_{b} \exp _{x_{k}}^{\tau_{1}}$ is the $\mathscr{L}_{b}$-exponential map defined in [29], taking $v$ to $\eta\left(\tau_{1}\right)$ with $\eta$ being the $\mathscr{L}_{b}$-geodesic with initial vector $v, J\left(v, \tau_{1}\right)=\operatorname{det} d\left(\mathscr{L}_{b} \exp _{x_{k}}^{\tau_{1}}\right)$ denotes the Jacobian of $\mathscr{L}_{b} \exp _{x_{k}}^{\tau_{1}}$ and $\Omega\left(\tau_{1}, k\right) \subset T_{x_{k}} M$ is a set which is mapped bijectively to $M$ up to a set of measure zero under the map $\mathscr{L}_{b} \exp _{x_{k}}^{\tau_{1}}$. In [28], we prove that the integrand

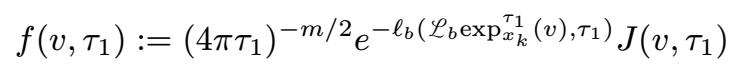

is non-increasing in $\tau_{1}$ for fixed $v$ and has the $\operatorname{limit}_{\tau_{1} \rightarrow 0} f\left(v, \tau_{1}\right)=\pi^{-m / 2} e^{-|v|^{2}}$. Together with $\Omega\left(\tau^{\prime}\right) \subset \Omega(\tau)$ for $\tau \leq \tau^{\prime}$ this yields an alternative proof of the monotonicity of the reduced volumes obtained in [29]. Moreover, it implies that the contribution to the reduced volume $\tilde{V}_{k}\left(\varepsilon_{k} r_{k}^{2}\right)$ coming from $\mathscr{L}_{b}$-geodesics with initial vector $|v|>\frac{1}{8} \varepsilon_{k}^{-1 / 2}$ can be bounded by

$$
\int_{|v|>\frac{1}{8} \varepsilon_{k}^{-1 / 2}} \pi^{-m / 2} e^{-|v|^{2}} d v \leq C e^{-\frac{1}{64 \varepsilon_{k}}} \rightarrow 0 \quad(k \rightarrow \infty),
$$

which completes the proof of Claim 1.

Claim 2. - The reduced volumes $\tilde{V}_{k}\left(t_{k}\right)$ are bounded below away from zero.

Proof. - Let us remark that $\tau=t_{k}$ corresponds to real time $t=0$. We assume that $k$ is large enough, so that $t_{k} \geq T / 2$. The idea behind the proof is to go from $\left(x_{k}, t_{k}\right)$ to some point $q_{k}$ at the real time $T / 2$ (i.e., $\tau=t_{k}-T / 2$ ) for which the reduced $\mathscr{L}_{b}$-distance $\ell_{b}\left(q_{k}, t_{k}-T / 2\right.$ ) is small. From the upper bound on $L_{b}$ from [29, Lemma 4.1], we see that for small $\tau$ it is possible to find a point $q_{k}(\tau)$ such that $\ell_{b}\left(q_{k}(\tau), \tau\right) \leq \frac{m}{2}$. On the other hand, combining the evolution equations for $\frac{\partial}{\partial \tau} \ell_{b}$ and $\triangle \ell_{b}$, we obtain

$$
\left.\frac{\partial}{\partial \tau}\right|_{\tau=\tau_{1}} \ell_{b}+\triangle \ell_{b} \leq-\frac{1}{\tau_{1}} \ell_{b}+\frac{m}{2 \tau_{1}}
$$

(in the barrier sense) and hence for the minimum of $\ell_{\min }(\tau)=\min _{q \in M} \ell_{b}(q, \tau)$

$$
\left.\frac{\partial}{\partial \tau}\right|_{\tau=\tau_{1}} \ell_{\min } \leq-\frac{1}{\tau_{1}} \ell_{\min }+\frac{m}{2 \tau_{1}}
$$

in the sense of difference quotients. The latter is obtained by applying the maximum principle to a smooth barrier. The inequality (8.10) shows that there is some point $q_{k}(\tau)$ with $\ell_{b}\left(q_{k}(\tau), \tau\right) \leq \frac{m}{2}$ for every $\tau$. As mentioned above, we choose $q_{k}$ at the real time $T / 2$ with $\ell_{b}\left(q_{k}, t_{k}-T / 2\right) \leq \frac{m}{2}$. Let $\eta:\left[0, t_{k}-T / 2\right] \rightarrow M$ be an $\mathscr{L}_{b}$-geodesic realizing this length. Moreover, let $\eta_{p}:\left[t_{k}-T / 2, t_{k}\right] \rightarrow M$ be $g(t=0)$-geodesics (i.e., $g\left(\tau=t_{k}\right)$-geodesics) from $q_{k}$ at time $\tau=t_{k}-T / 2$ to $p \in B^{q_{k}}:=B_{g\left(\tau=t_{k}\right)}\left(q_{k}, 1\right)=B_{g(t=0)}\left(q_{k}, 1\right)$ at time $\tau=t_{k}$. Since $|\mathrm{Rm}|$ is uniformly bounded for $t \in[0, T / 2]$, we get a uniform bound for $S$ along this family of curves. Since all the metrics $g(\tau)$ with $\tau \in\left[t_{k}-T / 2, t_{k}\right]$ are uniformly equivalent, we get a uniform upper bound for the $\mathscr{L}_{b}$-length of all $\eta_{p}$. From this, we see that the concatenations $\left(\eta \smile \eta_{p}\right):\left[0, t_{k}\right] \rightarrow M$ connecting $x_{k}$ to $p \in B^{q_{k}}$ have uniformly bounded $\mathcal{L}_{b}$-length, independent of $p$ and $k$. This gives a uniform bound $\ell_{b}\left(p, t_{k}\right) \leq C$, for all $p \in B^{q_{k}}$ and $k \in \mathbb{N}$ large enough. We can then estimate

$$
\tilde{V}_{k}\left(t_{k}\right)=\int_{M}\left(4 \pi t_{k}\right)^{-m / 2} e^{-\ell_{b}\left(q, t_{k}\right)} d V(q) \geq \int_{B^{q_{k}}}\left(4 \pi t_{k}\right)^{-m / 2} e^{-C} d V \geq C \inf _{q_{k} \in M} \operatorname{vol}\left(B^{q_{k}}\right),
$$

which is bounded below away from zero, independently of $k$. This proves Claim 2 . 
Since the backwards reduced volumes $\tilde{V}_{k}$ are non-increasing in $\tau$ (i.e., non-decreasing in real time $t$ ) according to Theorem 8.2, we obtain $\tilde{V}_{k}\left(t_{k}\right) \leq \tilde{V}_{k}\left(\varepsilon_{k} r_{k}^{2}\right)$ for $k$ large enough. But since $\tilde{V}_{k}\left(t_{k}\right)$ is bounded below away from zero by Claim 2 while $\tilde{V}_{k}\left(\varepsilon_{k} r_{k}^{2}\right)$ converges to zero with $k \rightarrow \infty$ by Claim 1, we obtain the desired contradiction that proves the theorem.

\section{Appendix}

\section{Commutator identities}

It is well-known that a $(p, q)$-tensor $B$ (i.e., a smooth section of the bundle $\left.\left(T^{*} M\right)^{\otimes p} \otimes(T M)^{\otimes q}\right)$ satisfies the following commutator identity in local coordinates $\left(x^{1}, \ldots, x^{n}\right)$ induced by a chart $\phi: U \rightarrow \mathbb{R}^{n}, U \subseteq M$,

$$
\left[\nabla_{i}, \nabla_{j}\right] B_{\ell_{1} \ldots \ell_{p}}^{k_{1} \ldots k_{q}}=\sum_{r=1}^{q} R_{i j m}^{k_{r}} B_{\ell_{1} \ldots \ell_{p}}^{k_{1} \ldots k_{r-1} m k_{r+1} \ldots k_{q}}+\sum_{s=1}^{p} R_{i j \ell_{s} m} B_{\ell_{1} \ldots \ell_{s-1} m \ell_{s+1} \ldots \ell_{p}}^{k_{1} \ldots k_{q}}
$$

Now, assume that we are given Levi-Civita connections for all $(p, q)$ tensors over $(M, g)$ and over $(N, \gamma)$. For a map $\phi:(M, g) \rightarrow(N, \gamma)$, there is a canonical notion of pull-back bundle $\phi^{*} T N$ over $M$ with sections $\phi^{*} V=V \circ \phi$ for $V \in \Gamma(T N)$. The Levi-Civita connection $\nabla^{T N}$ on $T N$ also induces a connection $\nabla^{\phi^{*} T N}$ on this pull-back bundle via

$$
\nabla_{X}^{\phi^{*} T N} \phi^{*} V=\phi^{*}\left(\nabla_{\phi_{*} X}^{T N} V\right), \quad X \in \Gamma(T M), V \in \Gamma(T N) .
$$

We obtain connections on all product bundles over $M$ with factors $T M, T^{*} M, \phi^{*} T N$ and $\phi^{*} T^{*} N$ via the product rule and compatibility with contractions. Take coordinates $x^{k}$ on $M$, $k=1, \ldots, m=\operatorname{dim} M$, and $y^{\mu}$ on $N, \mu=1, \ldots, n=\operatorname{dim} N$, and write $\partial_{k}$ for $\frac{\partial}{\partial x^{k}}$ and $\partial_{\mu}$ for $\frac{\partial}{\partial y^{\mu}}$. We get $\nabla_{i} \nabla_{j} V_{\kappa}-\nabla_{j} \nabla_{i} V_{\kappa}=R_{i j \kappa \lambda} V_{\lambda}$ with

$$
\begin{aligned}
R_{i j \kappa \lambda}(x) & =\left\langle\operatorname{Rm}\left(\partial_{i}, \partial_{j}\right) \phi^{*}\left(\partial_{\lambda}\right), \phi^{*}\left(\partial_{\kappa}\right)\right\rangle_{\phi^{*} T N}(x) \\
& =\left\langle{ }^{N} \operatorname{Rm}\left(\phi_{*} \partial_{i}, \phi_{*} \partial_{j}\right) \partial_{\lambda}, \partial_{\kappa}\right\rangle_{T N}(\phi(x)) \\
& ={ }^{N} R_{\mu \nu \kappa \lambda}(\phi(x)) \nabla_{i} \phi^{\mu}(x) \nabla_{j} \phi^{\nu}(x),
\end{aligned}
$$

where we used $\phi_{*} \partial_{i}=\nabla_{i} \phi^{\mu} \partial_{\mu}$. This allows to extend (A.1) to mixed tensors, for example

$$
\left[\nabla_{i}, \nabla_{j}\right] B_{\ell \lambda}^{k \kappa}=R_{i j p}^{k} B_{\ell \lambda}^{p \kappa}+R_{i j \ell p} B_{p \lambda}^{k \kappa}+R_{i j \varrho}^{\kappa} B_{\ell \lambda}^{k \varrho}+R_{i j \lambda \varrho} B_{\ell \varrho}^{k \kappa} .
$$

The standard example that will be used quite often is the following. The derivative $\nabla \phi$ of $\phi: M \rightarrow N$ is a section of $T^{*} M \otimes \phi^{*} T N$. Thus, the intrinsic second order derivative is built with the connection on this bundle, i.e., $\nabla_{i} \nabla_{j} \phi^{\lambda}=\partial_{i} \partial_{j} \phi^{\lambda}-\Gamma_{i j}^{k} \partial_{k} \phi^{\lambda}+{ }^{N} \Gamma_{\mu \nu}^{\lambda} \partial_{i} \phi^{\mu} \partial_{j} \phi^{\nu}$ and similar for higher derivatives. Using (A.2), we obtain

$$
\nabla_{i} \nabla_{j} \nabla_{\ell} \phi^{\beta}-\nabla_{j} \nabla_{i} \nabla_{\ell} \phi^{\beta}=R_{i j \ell p} \nabla_{p} \phi^{\beta}+{ }^{N} R_{\mu \nu \lambda}^{\beta} \nabla_{\ell} \phi^{\lambda} \nabla_{i} \phi^{\mu} \nabla_{j} \phi^{\nu} .
$$

There is also a different way to obtain these formulas, which is especially useful when $\phi$ is evolving and we also want to include time derivatives. We learned this from [20]. Here, we interpret $\nabla^{k} \phi$ as a $k$-linear $T N$-valued map along $\phi \in C^{\infty}(M, N)$ rather than as a section in $\left(T^{*} M\right)^{\otimes k} \otimes \phi^{*} T N$. Letting $\omega$ be any such $k$-linear $T N$-valued map along $\phi$, 
i.e., $\omega(x):\left(T_{x} M\right)^{\times k} \rightarrow T_{\phi(x)} N$, the covariant derivative $\nabla \omega$ is a $(k+1)$-linear $T N$-valued map along $\phi$, etc. The curvature tensor ${ }^{k} \mathrm{Rm}$ for $\omega$ can then be computed by

$$
\left({ }^{k} \operatorname{Rm}(X, Y) \omega\right)\left(X_{1}, \ldots, X_{k}\right)={ }^{N} \operatorname{Rm}(\nabla \phi(X), \nabla \phi(Y)) \omega\left(X_{1}, \ldots, X_{k}\right)
$$

$$
-\sum_{s=1}^{k} \omega\left(X_{1}, \ldots, \operatorname{Rm}(X, Y) X_{s}, \ldots, X_{k}\right) \text {. }
$$

Of course, this agrees with the definition above, where we used the bundle interpretation. Now, if $\phi$ is time-dependent, we simply interpret it as a map $\tilde{\phi}: M \times I \rightarrow N$ and interpret $\nabla^{k} \phi$ as $k$-linear $T N$-valued maps on $M \times I$ along $\tilde{\phi}$. The formalism stays exactly the same.

Note that $\frac{\partial}{\partial t}$ induces a covariant time derivative $\nabla_{t}$ (on all bundles over $M \times I$ ) that agrees with $\frac{\partial}{\partial t}$ for time-dependent functions. Choose coordinates $x^{i}$ for $M$ with

$$
\nabla_{t}\left(\frac{\partial}{\partial t}\right)=\nabla_{i}\left(\partial_{j}\right)=\nabla_{t}\left(\partial_{i}\right)=\nabla_{i}\left(\frac{\partial}{\partial t}\right)=0, \quad \forall i, j=1, \ldots, m
$$

at some base point $(p, t)$ in $M \times I$. Then, using (A.4), we obtain for $\omega=\nabla \phi$

$$
\begin{aligned}
\nabla_{t}\left(\nabla_{i} \nabla_{j} \phi\right) & =\nabla_{t}\left(\left(\nabla_{i} \omega\right)\left(\partial_{j}\right)\right)=\left(\nabla_{t} \nabla_{i} \omega\right)\left(\partial_{j}\right)=\left(\nabla_{i} \nabla_{t} \omega+{ }^{1} \operatorname{Rm}\left(\frac{\partial}{\partial t}, \partial_{i}\right) \omega\right)\left(\partial_{j}\right) \\
& =\nabla_{i}\left(\left(\nabla_{t} \omega\right)\left(\partial_{j}\right)\right)+{ }^{N} \operatorname{Rm}\left(\frac{\partial}{\partial t} \phi, \nabla_{i} \phi\right) \omega\left(\partial_{j}\right)-\omega\left({ }^{M \times I} \operatorname{Rm}\left(\frac{\partial}{\partial t}, \partial_{i}\right) \partial_{j}\right) \\
& =\nabla_{i} \nabla_{j} \frac{\partial}{\partial t} \phi+{ }^{N} \operatorname{Rm}\left(\frac{\partial}{\partial t} \phi, \nabla_{i} \phi\right) \nabla_{j} \phi .
\end{aligned}
$$

REMARK A.1. - If we also vary the metric $g$ on $M$ in time, we will get an additional term from the evolution of $\nabla_{i} \nabla_{j}$, namely $-\left(\frac{\partial}{\partial t} \Gamma_{i j}^{k}\right) \nabla_{k} \phi$. Note that $\frac{\partial}{\partial t} \Gamma$ is a tensor, while $\Gamma$ itself is not.

\section{REFERENCES}

[1] S. BAndo, Real analyticity of solutions of Hamilton's equation, Math. Z. 195 (1987), 93-97.

[2] S. Bernstein, Sur la généralisation du problème de Dirichlet II, Math. Ann. 69 (1910), 82-136.

[3] H.-D. CaO, X.-P. ZHU, A complete proof of the Poincaré and geometrization conjectures - application of the Hamilton-Perelman theory of the Ricci flow, Asian J. Math. 10 (2006), 165-492.

[4] B. Chow, S.-C. Chu, D. Glickenstein, C. Guenther, J. Isenberg, T. Ivey, D. Knopf, P. Lu, F. Luo, L. Ni, The Ricciflow: Techniques and applications: Part I: Geometric aspects, Mathematical Surveys and Monographs 135, Amer. Math. Soc., 2007.

[5] B. Chow, S.-C. Chu, D. Glickenstein, C. Guenther, J. Isenberg, T. Ivey, D. Knopf, P. Lu, F. Luo, L. Ni, The Ricci flow: Techniques and applications: Part II: Analytic aspects, Mathematical Surveys and Monographs 144, Amer. Math. Soc., 2008.

[6] B. Chow, D. Knopf, The Ricci flow: An introduction, Mathematical Surveys and Monographs 110, Amer. Math. Soc., 2004.

[7] B. Chow, P. Lu, L. Ni, Hamilton's Ricci flow, Graduate Studies in Math. 77, Amer. Math. Soc., 2006.

$4^{\mathrm{e}}$ SÉRIE - TOME $45-2012$ - No 1 
[8] T. H. Colding, W. P. Minicozzi II, Estimates for the extinction time for the Ricci flow on certain 3-manifolds and a question of Perelman, J. Amer. Math. Soc. 18 (2005), 561-569.

[9] T. H. Colding, W. P. Minicozzi II, Width and finite extinction time of Ricci flow, Geom. Topol. 12 (2008), 2537-2586.

[10] D. M. DeTurck, Deforming metrics in the direction of their Ricci tensors, J. Differential Geom. 18 (1983), 157-162.

[11] J. Eells, L. Lemaire, A report on harmonic maps, Bull. London Math. Soc. 10 (1978), $1-68$.

[12] J. Eells, L. Lemaire, Another report on harmonic maps, Bull. London Math. Soc. 20 (1988), 385-524.

[13] J. Eells, J. H. Sampson, Harmonic mappings of Riemannian manifolds, Amer. J. Math. 86 (1964), 109-160.

[14] C. Guenther, J. Isenberg, D. Knopf, Stability of the Ricci flow at Ricci-flat metrics, Comm. Anal. Geom. 10 (2002), 741-777.

[15] R. S. Hamilton, Three-manifolds with positive Ricci curvature, J. Differential Geom. 17 (1982), 255-306.

[16] R. S. Hamilton, The formation of singularities in the Ricci flow, in Surveys in differential geometry, Vol. II (Cambridge, MA, 1993), Int. Press, Cambridge, MA, 1995.

[17] P. Hartman, A. Wintner, On the local behavior of solutions of non-parabolic partial differential equations, Amer. J. Math. 75 (1953), 449-476.

[18] J. Jost, Riemannian geometry and geometric analysis, third ed., Universitext, Springer, 2002.

[19] B. Kleiner, J. Lott, Notes on Perelman's papers, Geom. Topol. 12 (2008), 2587-2855.

[20] T. Lamm, Biharmonic maps, Ph.D. Thesis, Albert-Ludwig-Universität Freiburg im Breisgau, 2005.

[21] A. Lichnerowicz, Propagateurs et commutateurs en relativité générale, Publ. Math. I.H.É.S. 10 (1961).

[22] B. List, Evolution of an extended Ricci flow system, Ph.D. Thesis, Albert-EinsteinInstitut, Berlin, 2005.

[23] J. Lotт, On the long-time behavior of type-III Ricci flow solutions, Math. Ann. 339 (2007), 627-666.

[24] C. Mantegazza, L. Martinazzi, A note on quasilinear parabolic equations on manifolds, to appear in Ann. Scuola Norm. Sup. Pisa Cl. Sci.

[25] J. Morgan, G. Tian, Ricciflow and the Poincaré conjecture, Clay Mathematics Monographs 3, Amer. Math. Soc., 2007.

[26] J. Morgan, G. Tian, Completion of the proof of the geometrization conjecture, preprint arXiv:0809.4040.

[27] R. MüLlER, Differential Harnack inequalities and the Ricciflow, EMS Series of Lectures in Mathematics, European Mathematical Society (EMS), Zürich, 2006.

[28] R. MÜLLER, The Ricci flow coupled with harmonic map heat flow, Ph.D. Thesis, ETH Zürich, 2009. 
[29] R. MÜLLER, Monotone volume formulas for geometric flows, J. reine angew. Math. 643 (2010), 39-57.

[30] J. Nash, The imbedding problem for Riemannian manifolds, Ann. of Math. 63 (1956), 20-63.

[31] G. Perelman, The entropy formula for the Ricci flow and its geometric applications, preprint arXiv:math/0211159.

[32] G. Perelman, Finite extinction time for the solutions to the Ricci flow on certain threemanifolds, preprint arXiv:math/0307245.

[33] G. Perelman, Ricci flow with surgery on three-manifolds, preprint arXiv:math/0303109.

[34] H. Poincaré, Cinquième complément à l'Analysis Situs, Rend. Circ. Mat. Palermo 18 (1904), 45-110.

[35] M. Reed, B. Simon, Methods ofmodern mathematical physics. IV. Analysis of operators, Academic Press Inc., 1978.

[36] O. S. Rothaus, Logarithmic Sobolev inequalities and the spectrum of Schrödinger operators, J. Funct. Anal. 42 (1981), 110-120.

[37] O. C. Schnürer, F. Schulze, M. Simon, Stability of Euclidean space under Ricci flow, Comm. Anal. Geom. 16 (2008), 127-158.

[38] W.-X. SHI, Deforming the metric on complete Riemannian manifolds, J. Differential Geom. 30 (1989), 223-301.

[39] M. Simon, Deformation of $C^{0}$ Riemannian metrics in the direction of their Ricci curvature, Comm. Anal. Geom. 10 (2002), 1033-1074.

[40] M. Struwe, Geometric evolution problems, in Nonlinear partial differential equations in differential geometry (Park City, UT, 1992), IAS/Park City Math. Ser. 2, Amer. Math. Soc., 1996, 257-339.

[41] T. C. TAo, Perelman's proof of the Poincaré conjecture: a nonlinear PDE perspective, preprint arXiv:math/0610903.

[42] W. P. Thurston, Three-dimensional manifolds, Kleinian groups and hyperbolic geometry, Bull. Amer. Math. Soc. (N.S.) 6 (1982), 357-381.

[43] P. Topping, Lectures on the Ricci flow, London Math. Soc. Lecture Note Series 325, Cambridge Univ. Press, 2006.

[44] M. B. Williams, Results on coupled Ricci and harmonic map flows, preprint arXiv:1012.0291.

(Manuscrit reçu le 23 juillet 2010; accepté, après révision, le 12 septembre 2011.)

\footnotetext{
Reto MÜLLER

Imperial College London

Department of Mathematics

London SW7 2AZ, UK

E-mail: r.mueller@imperial.ac.uk
}

4 ${ }^{\mathrm{e}}$ SÉRIE - TOME $45-2012$ - No 1 